\title{
Spatiotemporal solidification of $\alpha$-synuclein inside the liquid droplets
}

Soumik Ray ${ }^{1, \dagger}$, Debdeep Chatterjee ${ }^{1, \dagger}$, Semanti Mukherjee ${ }^{1}$, Komal Patel ${ }^{1}$, Jaladhar Mahato ${ }^{2}$, Sangram Kadam $^{1}$, Rakesh Krishnan ${ }^{1}$, Ajay Singh Sawner ${ }^{1}$, Manisha Poudyal ${ }^{1}$, G Krishnamoorthy ${ }^{3}$, Arindam Chowdhury $^{2}$, Ranjith Padinhateeri ${ }^{1}$ and Samir K. Maji ${ }^{*}$

${ }^{1}$ Department of Biosciences and Bioengineering, IIT Bombay, Powai, Mumbai-400076, India ${ }^{2}$ Department of Chemistry, IIT Bombay, Powai, Mumbai 400076, India

${ }^{3}$ Department of Biotechnology, Anna University, Chennai 600025, India

${ }^{\dagger}$ Contributed equally

*Correspondence: Prof. Samir K. Maji, Department of Biosciences and Bioengineering, IIT Bombay, Powai, Mumbai 400 076, India, Tel: + (91-22) 2576-7774, Fax: + (91-22) 2572 3480. Email: samirmaji@iitb.ac.in KEYWORDS: Liquid-liquid phase separation; liquid-to-solid transition; $\alpha$-synuclein. 

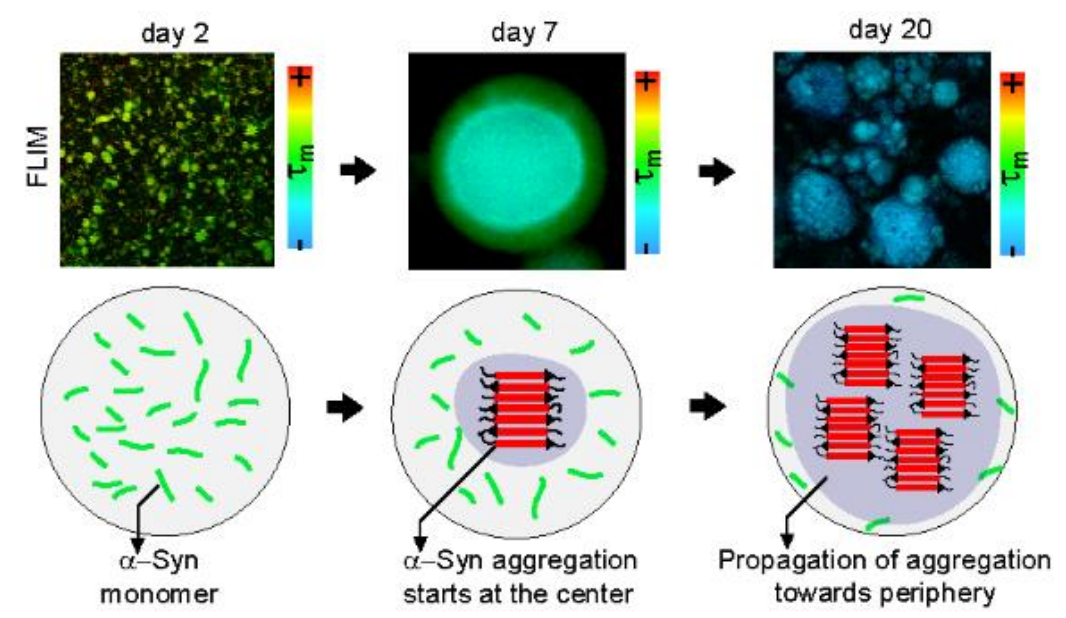

ABSTRACT: Liquid-liquid phase separation (LLPS) and subsequent liquid-to-solid transition is implicated in membraneless organelles formation as well as disease associated protein aggregation. However, how liquid-to-solid transition is initiated inside a liquid droplet remains unclear. Here, using studies at single droplet resolution, we show that liquid-to-solid transition of $\alpha$-synuclein ( $\alpha$-Syn) liquid droplets is associated with significant changes in the local microenvironment as well as secondary structure of the protein, which is prominently observed at the center of the liquid droplets. With the ageing of liquid droplets, the "structured" core at the center gradually expands and propagates over entire droplets. Further, during droplet fusion, smaller, homogeneous droplets progressively dissolve and supply proteins to the larger, heterogeneous droplets containing solid-like core at their center. The present study will significantly help to understand the physical mechanism of LLPS and liquid-to-solid transition in biological compartmentalization as well as in protein aggregation associated with human neurodegenerative disorders. 


\section{Introduction}

Liquid-liquid phase separation (LLPS) by various biomolecules such as protein and nucleic acids has been shown to be crucial for the formation of membrane-less organelles to compartmentalize biomolecules in liquid-like condensates ${ }^{1-11}$. The LLPS is involved in multiple cellular functions such as concentrating cellular components into organizational hubs ${ }^{1-10,12-17}$, enriching component molecules for specific biochemical reactions with regulated kinetics and high specificity ${ }^{18-25}$ and sequester proteins in response to specific physico-chemical stimuli ${ }^{26-30}$. The major advantage of membrane-less organelle formation is their ability to assemble, disassemble, fuse and exchange their constituents with the surrounding according to the functional requirement of the cell ${ }^{1,4,31-33}$. Proteins with intrinsically disorder regions (IDRs) as well as closely related low complexity domains (LCDs) have been shown to undergo transient, multivalent interactions responsible for biomolecular condensation ${ }^{12,34-40}$. Apart from various important functional roles of LLPS, liquid droplets formed by many IDR containing proteins have shown to undergo liquid-to-solid transition during time ${ }^{12,13,41-46}$. Liquid-to-solid phase transition is a consequence of strong interaction between protein molecules owing to their entanglement, rapid vitrification and amyloid fibril formation (and network formation through lateral association $)^{22}$. Once solidified, the droplets can no longer fuse, deform or exchange their constituents with the surroundings ${ }^{12,41-46}$.

Notably, liquid-to-solid transition of LLPS is often associated with many biological functions of the host organism $^{7,47-49}$. Interestingly, such liquid droplets and bimolecular condensates could contain a solid fraction as a sub-structure ${ }^{22}$. However, the ratio of solid and liquid fractions could be actively regulated by cells to optimize functionality of such heterogeneous liquid droplets ${ }^{22}$. Apart from biological functions, liquid-to-solid transition is also associated with toxic oligomer and fibril formation associated with human diseases $^{50-53}$. For example; LLPS of FUS ${ }^{41,42}$ and hnRNPA1 $1^{12}$ is important for formation and assembly of stress granules; but deregulation of LLPS of these proteins leads to aberrant solidification and fibril formation in amyotrophic lateral sclerosis (ALS). The balance between functional versus aberrant LLPS of proteins could also be modulated by factors such as in cell environment, disease associated mutations and post-translational modifications in proteins ${ }^{12,36,39,41,42,54-57}$. Natively unstructured proteins associated with neurodegenerative disorders have also been reported to undergo LLPS and liquid-to-solid transition ${ }^{41-44}$. In this direction, recently, we showed that $\alpha$-synuclein ( $\alpha$-Syn) could also phase separate into liquid droplets both in vitro and in cell ${ }^{55}$. Interestingly, these droplets act as nucleation sites for $\alpha$-Syn aggregation and undergo a viscoelastic transition with time (from liquid-like to solid-like). The droplets eventually mature into an amyloid fibril containing hydrogel in vitro ${ }^{55,58,59}$. 
Although liquid-to-solid transition is prevalent in biological LLPS, but the mechanism of liquid-to-solid transition and the location where solidification begins is unknown. Rigidification within the droplets could start at the center, periphery, or could be completely at random locations. In this study, we used fluorescence lifetime imaging (FLIM), single point Fourier transform infra-red microscopy (FTIRM) imaging, spatially resolved Förster resonance energy transfer (FRET) microscopy and fluorescence recovery after photobleaching (FRAP) to unveil how liquid-to-solid transition is initiated inside $\alpha$-Syn liquid droplets. Our data revealed that a solid-like core forms at the center of liquid droplets, which gradually progresses towards the periphery until the entire droplet is rigidified. We further show that the formation of the solidlike core is due to secondary structural transition of $\alpha$-Syn into $\beta$-sheet rich amyloid fibrils via $\alpha$-helix rich intermediate formation. Furthermore, analyses of droplet growth via fusion revealed that the droplet containing a structured core grows in the expense of smaller droplets without structured core. Together, our findings prove that liquid-to-solid transition of $\alpha$-Syn LLPS follows a specific pattern (center $\rightarrow$ periphery), which could be a generic phenomenon for many natively unstructured proteins containing LCDs/IDRs that undergo such aberrant phase transitions.

\section{Results}

\section{Fluorescence life-time imaging (FLIM) shows changes in $\alpha$-Syn microenvironment at the center of liquid droplets, which extends towards the periphery during liquid-to-solid transition}

To delineate the changes in protein property during liquid to solid transition, we phase separated $\alpha$-Syn using a condition ${ }^{55}(200 \mu \mathrm{M} \alpha-S y n+10 \%$ (w/v) PEG-8000 in $20 \mathrm{mM}$ sodium phosphate buffer, $\mathrm{pH}$ 7.4), where $\alpha$-Syn phase separation was slow compared to high salt conditions ${ }^{60}$. Immediately after their formation and during liquid-to-solid transition, we employed FLIM studies to delineate spatiotemporal change of protein microenvironment inside the droplets. Important to note, that FLIM studies can be used to delineate the mean fluorescence lifetime $\left(\tau_{\mathrm{m}}\right)$ of proteins independent of their concentrations (or fluorophore concentration) ${ }^{61,62}$. Moreover, due to the involvement of central hydrophobic domain (NAC domain) of $\alpha$-Syn (61-95 amino acids) in LLPS $^{55}$ as well as in aggregation ${ }^{63}$; we used 74-Cysteine- $\alpha$-Syn mutant (74C- $\alpha$-Syn) labeled with fluorescein-5-maleimide ${ }^{55}$ for FLIM measurements (Supplementary Fig 1-2). Fluorescein labeled $\alpha$-Syn showed observable liquid droplets at day $2(\mathrm{~d} 2)^{55}$ with frequent fusion event, which was visible under fluorescence microscope, suggesting their liquid-like nature (Fig 1a and Supplementary Fig. 2). For FLIM imaging, the central plane along the Z-axis (depth) was chosen (Fig 1b, Left panel). To probe the spatial dependence, we collected lifetime data at the center (C) and on three concentric circles (inner circle, IC; outer circle, OC and periphery, P) equally spaced from each other (Fig 1b, Right panel). In addition, we acquired similar data from equally spaced focal planes (plane $1 \rightarrow 3$ ) 
along the Z-axis to analyze the droplets along their depth (Z-axis) (Fig 1c). From the FLIM images (Fig 1d, Left panel), time-resolved fluorescence intensity decay profiles from all four regions (C, IC, OC and P) on the central plane were acquired (Fig 1d, Middle panel), which could be satisfactorily fit to biexponential decay functions (Supplementary methods) to obtain two lifetimes — one long fluorescence lifetime $\left(\Delta_{\mathrm{m}}\right)$ and one short fluorescence lifetime $\left(\lambda_{\mathrm{m}}\right)$. Interestingly, our observations show that at $\mathrm{d} 2$, there were no significant differences between the two lifetimes $\left(\Delta_{\mathrm{m}}, \lambda_{\mathrm{m}}\right)$ across different regions $(\mathrm{C}$, IC, OC and P) of the droplets (Fig 1d, Right panel). Therefore, the local microenvironment of $\alpha$-Syn was identical throughout the droplets at early stages of LLPS (d2). In contrast, similar analysis confirmed a significant difference in the $\Delta_{\mathrm{m}}$ for different regions at d5 (C bearing the lowest $\Delta_{\mathrm{m}}=2.74 \mathrm{~ns}$ and $\mathrm{P}$ bearing the highest $\Delta_{\mathrm{m}}=7.06 \mathrm{~ns}$ ). IC and OC showed $\Delta_{\mathrm{m}}$ of 3.03 and $5.56 \mathrm{~ns}$, respectively (Fig 1d, Right panel). Similar trend was observed for d10 droplets. Strikingly, d15 droplets showed an overall decrease in the $\Delta_{\mathrm{m}}$ values across all positions on the droplet $\left(\Delta_{\mathrm{m}}\right.$ of C, IC, OC and P were calculated to be $1.7 \mathrm{~ns}, 2.03 \mathrm{~ns}$, $2.52 \mathrm{~ns}$ and $2.97 \mathrm{~ns}$, respectively). $\Delta_{\mathrm{m}}$ was further decreased for d20 droplets. However, the data unveiled no significant differences with respect to different regions of the droplets at d20 (Fig 1d, Right panel). Additionally, the $\lambda_{\mathrm{m}}$ was found to be similar ( $\left.1.00 \mathrm{~ns}\right)$ for different regions and no change was noted during the timescale (d5-d20) of our experiment. The amplitudes of $\lambda_{\mathrm{m}}$ and $\Delta_{\mathrm{m}}$ were almost equal $(50 \%)$ in most of the cases. Since we did not know the origin of the two lifetimes, we calculated the mean fluorescence lifetimes $\left(\tau_{\mathrm{m}}\right)$ as a comparative measure of our analyses (Fig 1e). Our results revealed that there is no significant difference between $\tau_{\mathrm{m}}(\sim 4 \mathrm{~ns}$; denoted with green pixels) across different regions of the droplets at d2 (Fig 1e-f), which is similar to soluble, monomeric protein $\left(\tau_{\mathrm{m}}=4.1 \pm 1 \mathrm{~ns}\right)$ (Supplementary Fig. 3). This observation indicated towards the monomeric state of $\alpha$-Syn inside the liquid droplets at d2. In contrast, independently prepared fluorescence-5-maleimide labeled fibrils showed significantly lower lifetime values ( $\tau_{\mathrm{m}} \sim 1 \mathrm{~ns}$; denoted with blue colored pixels) (Supplementary Fig. 3), which is consistent with previously observed $\alpha$-Syn fibrils in C.elegans ${ }^{64}$. When FLIM images of droplets at d5 and d10 were acquired; strikingly, the data showed lower $\tau_{\mathrm{m}}(\sim 2 \mathrm{~ns}$; denoted with blue colored pixels) at the center $(C)$ of the droplets compared to the periphery $(\mathrm{P})\left(\tau_{\mathrm{m}} \sim 4 \mathrm{~ns}\right.$; denoted with green colored pixels, similar to monomeric protein). However, the IC and OC regions showed intermediate values of $\tau_{\mathrm{m}}(\sim 2.15$ and $\sim 3.75$ ns, respectively) (Fig 1e-f). FLIM measurements along different Z-planes for d5 droplets further revealed a steady increase of the lifetime for planes progressively farther from the central plane, indicating that the vast majority of proteins were aggregated in the central core region of the droplet (Supplementary Fig. 3). During ageing (at d15 and d20), the $\tau_{\mathrm{m}}$ of the entire droplet was, however, lowered and became close to $\sim 1$ ns (similar to $\alpha$-Syn fibril state, Fig 1e-f) suggesting a homogenous phase where proteins are mostly aggregated over entire droplets. 


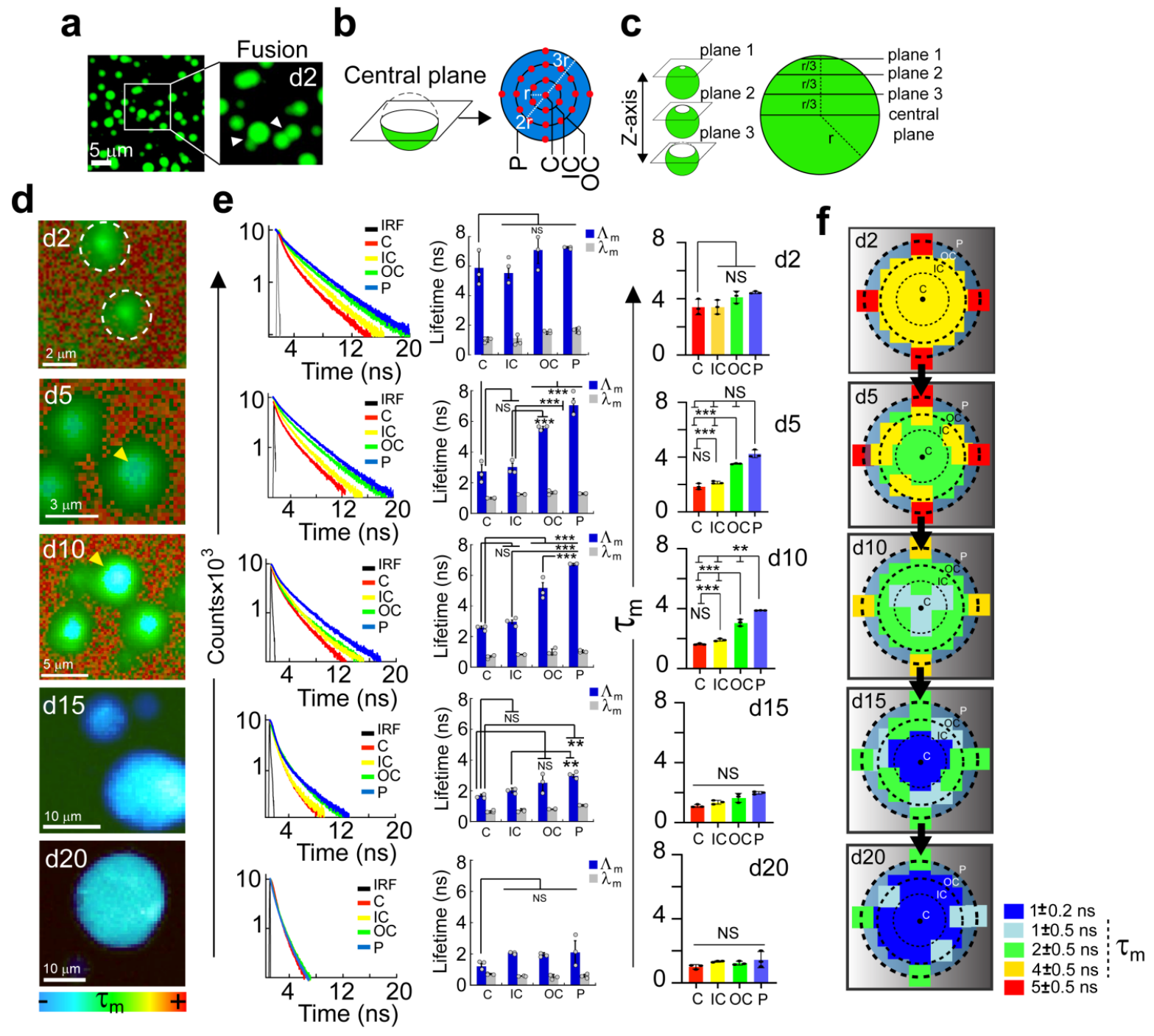

Figure 1: Changes in fluorescence lifetime initiates at the center and progresses towards the periphery of $\alpha$ Syn phase-separated droplets during liquid-to-solid transition

a. Liquid droplets formed by fluorescein-5-maleimide labeled $74 \mathrm{C}-\alpha-$ Syn at day 2 (d2). The inset indicates the fusion events between the droplets confirming their liquid-like state. $\mathbf{b}$. Schematic representation of the data-points selected from the central plane of a droplet for FLIM studies. The data-points are denoted as red circles $(\bullet)$. c. Schematic showing analysis of droplets along the depth (Z-axis). Apart from the central plane, three other planes (plane 1,2 and 3) along the Z-axis of the droplets are also imaged for FLIM to examine the distribution of lifetimes along the depth. The planes are equally spaced with a distance of $r / 3$ with radius of the droplet is $r$. d. Left panel: Representative color coded original FLIM images confirming the presence of the fluorescein-5-maleimide labeled $74 \mathrm{C}-\alpha-$-Syn droplets at various time points (d2-d20) during liquid-to-solid transition. The color spectrum represents the range of overall mean lifetime $\left(\tau_{\mathrm{m}}\right)$ (blue being the lowest and red being the highest). The images are acquired from the central plane. The white dashed circles mark the boundary of the liquid droplets at $\mathrm{d} 2$. Middle panel: Time resolved fluorescence intensity decay obtained from different regions (C-Center, IC-Inner Circle, OC-Outer circle and P-Periphery) of the droplets at various time points $(\mathrm{d} 2-\mathrm{d} 20)$. The black line represents instrument response 
function (IRF). The data-points are chosen as described in panel $\boldsymbol{b}$. Representative spectra are shown. The experiment is performed three times with similar observations. Right panel: The long $\left(\Delta_{\mathrm{m}}\right)$ and short $\left(\lambda_{\mathrm{m}}\right)$ lifetimes obtained from the intensity decay curved are plotted for C-Center, IC-Inner circle, OC-Outer circle and P-Periphery at different time-points $(\mathrm{d} 2, \mathrm{~d} 5, \mathrm{~d} 10, \mathrm{~d} 15$ and $\mathrm{d} 20)$. The data represents mean \pm S.D. e. Corresponding mean lifetimes $\left(\tau_{\mathrm{m}}\right)$ obtained from intensity decay curve at different regions (C-Center, IC-Inner circle, OC-Outer circle and P-Periphery) of the phase-separated droplets are plotted for different time-points (d2, d5, d10, d15 and d20). The data represents mean \pm S.D. The statistical significance (d-e) is calculated using one way ANOVA followed by Student-Newman-Keuls (SNK) post hoc test with a 95\% confidence interval for $n=3$ independent experiments. f. Schematic representation of a lifetime map (SRM) of the droplets for different time points (d2-d20) obtained from the calculated $\tau_{\mathrm{m}}$ values for each region $(\mathrm{C}, \mathrm{IC}, \mathrm{OC}, \mathrm{P})$ is shown. Each data-point is represented as a color-coded box $(\boldsymbol{\square})$ with respect to the obtained $\tau_{\mathrm{m}}$. Black dot $(\bullet)$ represents the center of the droplet and black dashed circles represent the boundary of IC, OC and P.

We found similar results showing the formation of a solid-like core with reduced lifetime at $\mathrm{d} 5$, which gradually propagated towards the periphery till d20 when we performed FLIM experiments with sample prepared in $2 \mathrm{ml}$ Eppendorf tubes. Further, protein concentration also remained same after 20 days incubation in microscopy slides due to incubation in moist chamber and the FLIM results was not largely affected by sedimentation/wetting of the droplets on glass surface (we used only spherical droplet for the measurements) (Supplementary Fig. 4-6). Some heavier droplets at d10 onwards did show interaction with the surface at the bottom of the slide and showed a distorted (ring-like) lifetime distribution (Supplementary Fig. 6).

Collectively, our data showed that the local microenvironment of $\alpha$-Syn was homogeneous throughout the droplets at early stages of LLPS with $\alpha$-Syn of higher $\tau_{\mathrm{m}}$ (Fig 1f). However, it began to change at the center of the droplets first and at later time-points, entire droplets became homogeneous in terms of reduced $\tau_{\mathrm{m}}$ values (Fig 1f) similar to fibrils.

\section{Conformational transition of $\alpha$-Syn starts at the center and propagates towards the periphery of the phase-separated droplets}

Subsequently, to probe the secondary structure of $\alpha$-Syn at different regions in the droplets during liquidto-solid transition, we employed Fourier transform infra-red microscopy (FTIRM) imaging in a singlepoint mode (Supplementary methods). This was done to probe whether the dynamic progression of the altered local microenvironment (Fig 1) is due to conformational transition of $\alpha$-Syn associated with aggregation. Unlabeled wild-type (WT) $\alpha$-Syn liquid droplets were imaged using FTIRM at various timepoints (d2-d20) (Fig 2a). The data-points were chosen at C, IC, OC and P of the microscopic image of the central plane of the droplet and corresponding FTIR spectra were recorded (Fig 2b-c and Supplementary Fig. 7). The obtained spectra were deconvoluted and the individual peaks were assigned for specific secondary structures and their abundance (Supplementary Fig. 7-8). To make sure deconvolution does not affect the signal to noise ratio, water subtraction from each of our spectra by taking the appropriate 
buffer as a control was performed. In our case, we did not find any major peaks corresponding to artifacts or water vapor in the region beyond $1700 \mathrm{~cm}^{-1}$ (Fig $2 \mathbf{c}$ ). Some small peaks were observed which may be due to the side chain functional groups from acidic amino acids and carbonyls.

Our result revealed identical signature for all regions (C, IC, OC, P) with the highest intensity peak at $1648 \mathrm{~cm}^{-1}$, corresponding to the random coil (RC) conformation in $\alpha$-Syn droplet at d2 (Fig 2c and Supplementary Fig. 7-8). The individual peaks of the deconvoluted spectra further confirmed that the FTIR signature resembled soluble, monomeric $\alpha$-Syn (Supplementary Fig. 7). Interestingly, unlike the early stages of LLPS (d2); at d5, the regions OC and P showed a clear difference in their respective FTIR spectra compared to C and IC (Fig 2c and Supplementary Fig. 7), which was consistent with the lifetime data (Fig 1). While OC and P showed mostly RC conformation, C and IC showed presence of $\alpha$-helical conformation (Fig 2c and Supplementary Fig. 7-8). In line with previous reports, this suggested that $\alpha-$ Syn LLPS and subsequent aggregation is mediated by $\alpha$-helical oligomeric intermediate ${ }^{55,65}$. At d10; C, IC and OC exhibited $\beta$-sheet and $\alpha$-helical structures as major conformations (Fig 2c and Supplementary Fig. 7-8). The periphery (P) of the droplets at d10 still showed majorly RC conformation (Fig $2 \mathbf{c}$ and Supplementary Fig. 7-8). At d15, FTIR spectra from C and IC had two major peaks corresponding to $\beta$ sheet and cross $\beta$-sheet structure (Fig 2c and Supplementary Fig. 7-8). OC showed both RC and $\beta$-sheet; while P showed $\beta$-sheet and RC conformations (Fig 2c and Supplementary Fig. 7-8). The d10 and d15 data clearly indicated that structural transition leading to aggregation was initiated at the center. At d20, the entire droplets were found to exhibit only $\beta$-sheet and cross $\beta$-sheet structures (Fig 2c and Supplementary Fig. 7-8) indicating the progression of aggregation towards the periphery consistent with our FLIM data. The sudden increase in the \% abundance of $\alpha$-helical conformation (Supplementary Fig. 8) at the periphery (P) at d20 might be due to the recruitment of fresh molecules and their further conversion into $\alpha$-helix rich state after interacting with $\beta$-sheet rich droplets. The $\%$ abundance of the secondary structures and FTIR peak assignments at different spatial locations of the droplets during liquid-to-solid transition are provided in Supplementary table 2 and 3. Notably, a very similar method using Raman microscopy has been recently employed to calculate the absolute concentrations of ataxin-3 protein inside a single droplet ${ }^{66}$ further supporting the robustness of our FTIRM experiments. 
a

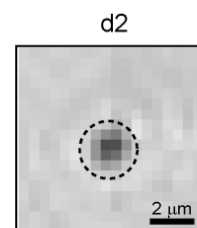

d5
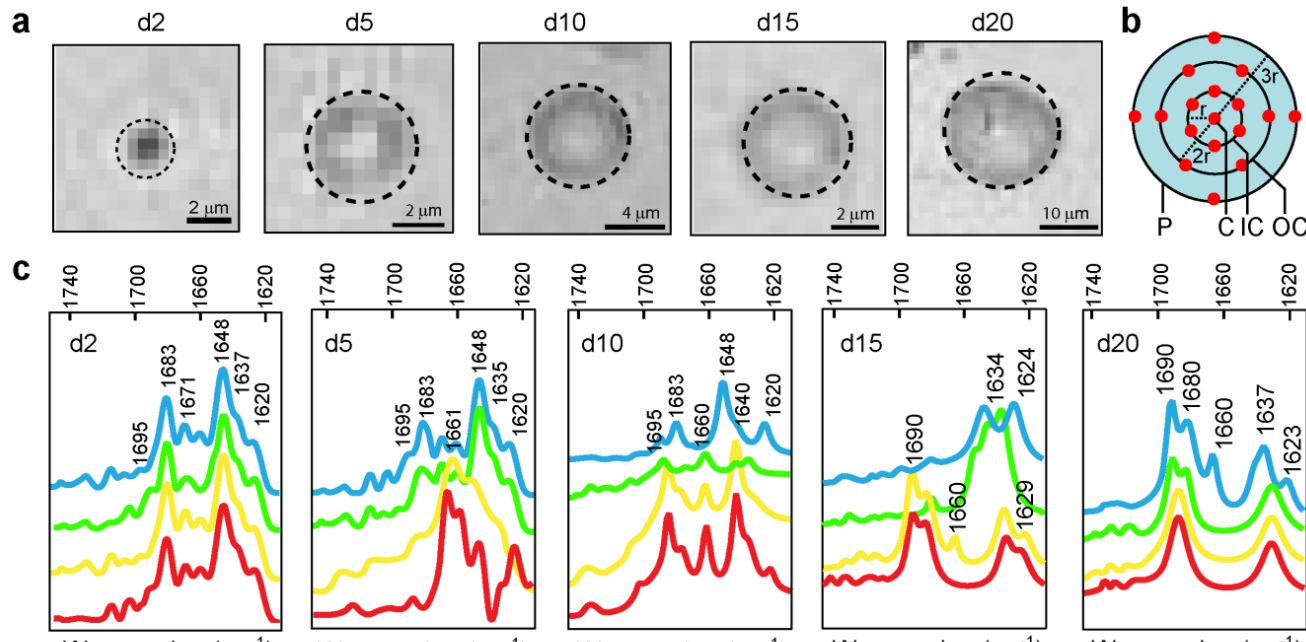

Wavenumber $\left(\mathrm{cm}^{-1}\right)$

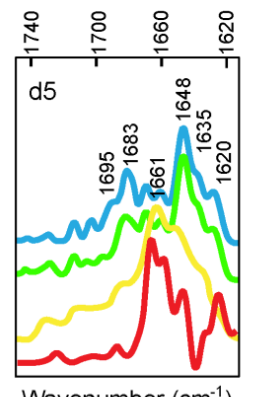

Wavenumber $\left(\mathrm{cm}^{-1}\right)$

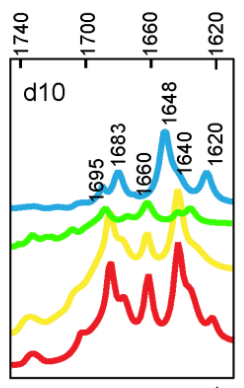

Wavenumber $\left(\mathrm{cm}^{-1}\right)$

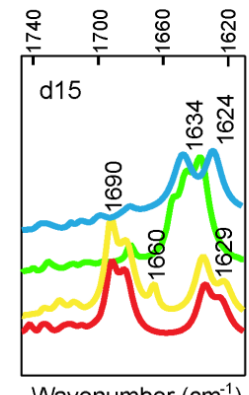

Wavenumber $\left(\mathrm{cm}^{-1}\right)$
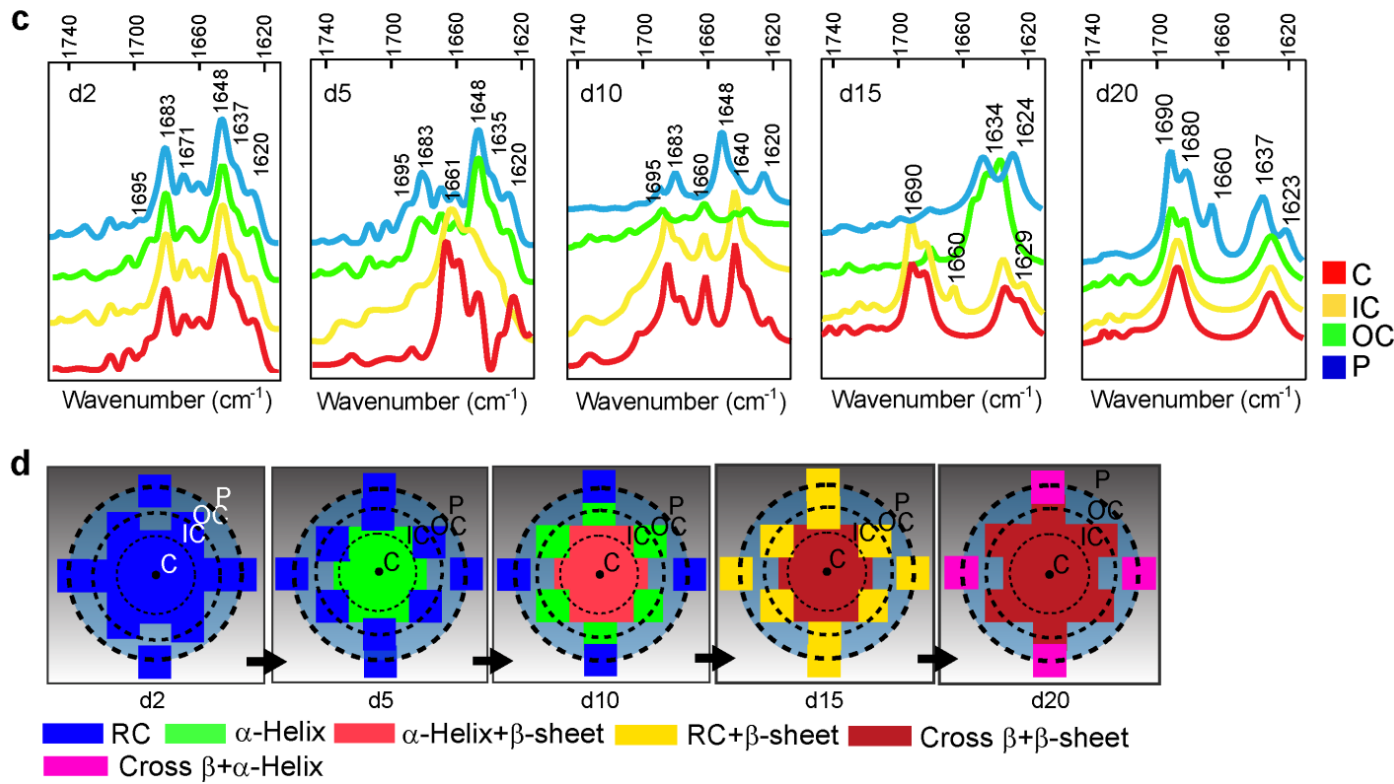

Figure 2: Position specific secondary structural transition in individual $\alpha$-Syn phase-separated droplets during liquid-to-solid transition

a. FTIRM images from the central plane of phase-separated $\alpha$-Syn droplets at different time-points (d2-d20) during liquid-to-solid transition. Black dashed circle represents the boundary of the droplet. The size of one single pixel was of $0.5 \mu \mathrm{m}$ length. Representative images are shown. The experiment is performed three times with similar observations. b. Schematic representation of the data-points selected from the central plane of a droplet for FTIRM studies. The data-points are denoted as red dots $(\bullet)$. c. Corresponding FTIR spectra obtained from different regions (C-Center, IC-Inner Circle, OC-Outer circle and P-Periphery) of the droplets are plotted for each time point (d2d20). One representative FTIR spectra for each data-point is shown. The respective wavenumber $\left(\mathrm{cm}^{-1}\right)$ for every peak in each spectrum is denoted. The experiment is carried out three times with similar results. d. Schematic representation depicting a spatially resolved secondary structural (based on deconvolution and \% secondary structure calculation) map (SRM) of the droplets for different time during incubation (d2-d20). Each data-point, from different regions, is represented by a color-coded box ( $\boldsymbol{\square})$ with respect to the obtained \% secondary structural majority. Black dot $(\bullet)$ represents the center and black dashed circles represent the boundary of IC, OC and P.

In essence, our spatially-resolved FTIRM measurements on single droplets suggested that $\alpha$-Syn liquidto-solid transition was accompanied by conformational transition ( $\mathrm{RC} \rightarrow \alpha$-helix $\rightarrow \beta$-sheet), which initiates at the center and then gradually covers the entire droplet (Fig $\mathbf{2 d}$ ).

\section{Single droplet FRET showed stronger intermolecular interaction at the center promoting liquid to solid transition $\alpha$-Syn liquid droplets}

Next, to further probe that the solid-like core formation at the center was due to $\alpha$-Syn aggregation, we performed spatially as well as spectrally resolved FRET $^{55}$ for single $\alpha$-Syn liquid droplets during liquid- 
to-solid transition (Supplementary Fig. 9). We chose fluorescein-5-maleimide labeled (10\% v/v) 74C$\alpha$-Syn as donor $(D)$ and rhodamine-C2-maleimide labeled (10\% v/v) 74C- $\alpha$-Syn as acceptor $(A)$ for our microscopy based FRET study at single droplet resolution ${ }^{55}$. The $74^{\text {th }}$ position was chosen to delineate intermolecular interactions of the NAC domain responsible for protein aggregation ${ }^{55,63}$ in a location specific manner inside the droplets. Crowding (10\% w/v PEG-8000) induced $\alpha$-Syn droplets were generated using an equimolar $(200 \mu \mathrm{M})$ concentration of $D$ and $A$. The spectral images (Supplementary methods) of liquid droplets containing both $D$ and $A$ were analyzed at C, OC, IC and P regions (Fig 3a-b). At d2, we observed similar extent of apparent FRET efficiency ${ }^{55}$ as evident from the $I E F_{E T}$ values $(\sim 1.2)$ at different locations (C, IC, OC, P) indicating the similar extent of intermolecular interaction throughout the droplets at early stages of LLPS (Fig 3c-d). Strikingly, at d5, we found that the apparent FRET efficiency $\left(I E F_{E T}\right)$ was highest at the center $\left(\mathrm{C}, I E F_{E T} \approx 2.4\right)$ and progressively lower towards the periphery $\left(\mathrm{P}, I E F_{E T} \approx 1.4\right)($ Fig 3c-d). This clearly indicated that the solid-like core at the center forms due to interdomain interaction of the NAC region, which eventually results in amyloid aggregation. At d20, all the regions $(\mathrm{C}, \mathrm{IC}, \mathrm{OC}$ and $\mathrm{P})$ showed similar $I E F_{E T}$ values $\left(I E F_{E T} \approx 2.4\right)($ Fig 3c-d) however with the overall increased magnitude of the $I E F_{E T}$ (Fig 3d). The data clearly indicates that higher extent of intermolecular interaction at the center leads to liquid to solid-transition, which eventually populates the entire droplet.
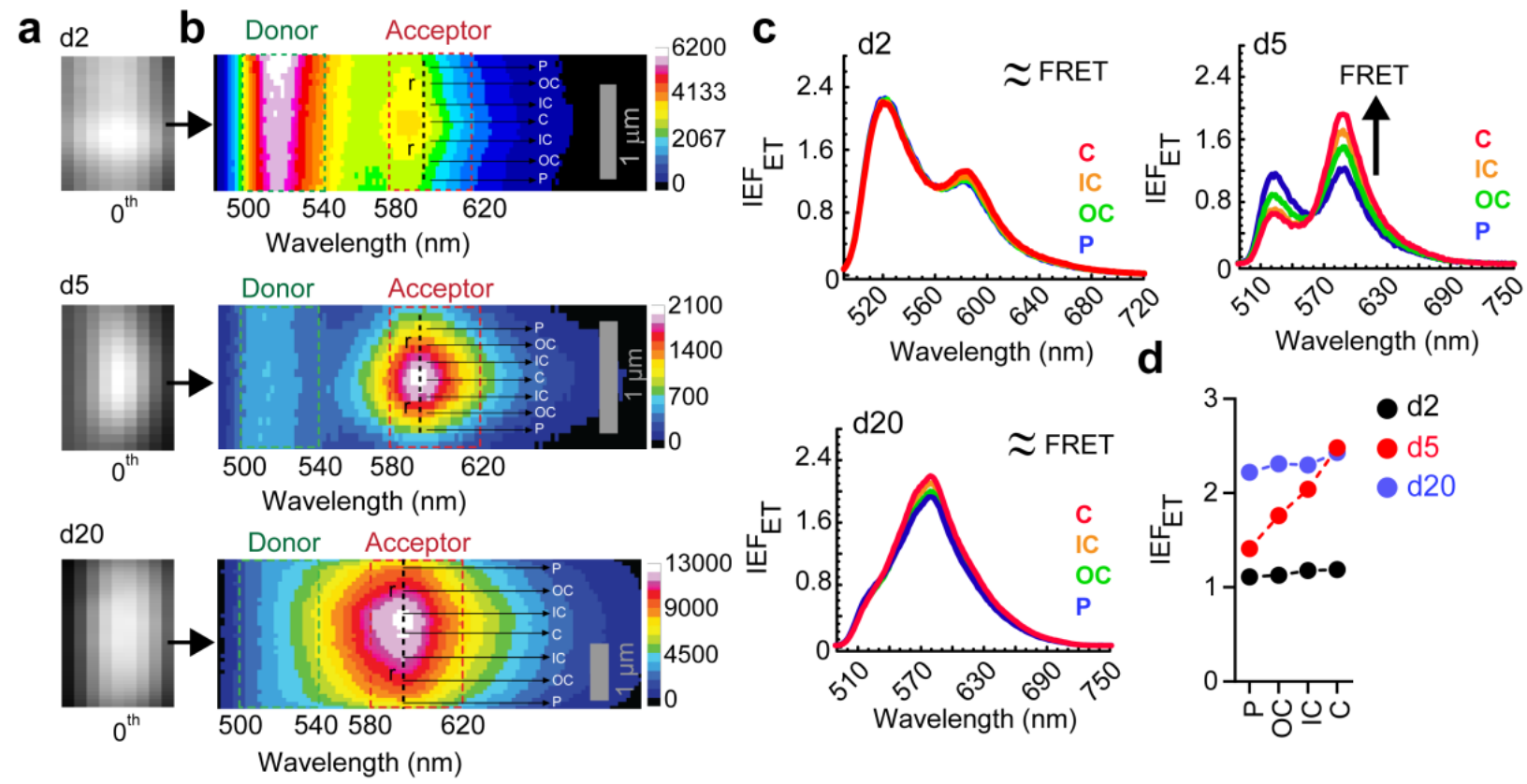

Figure 3: Intermolecular interaction responsible for protein aggregation initiates at the center of $\alpha$-Syn phase-separated droplets during liquid-to-solid transition

a. The $0^{\text {th }}$ order images of $\alpha$-Syn liquid droplets at various time points (d2, d5 and d20) during liquid-to-solid transition are shown. From these images, the spectrally resolved images are obtained. b. Spectrally resolved microscopic images of $\alpha$-Syn liquid droplets at $\mathrm{d} 2, \mathrm{~d} 5$ and d20, containing both fluorescein-5-maleimide (donor, $D$ ) and 
rhodamine-C2-maleimide (acceptor, $A$ ) labeled 74C- $\alpha$-Syn. The X-axis represents wavelength (nm) and Y-axis represents distance $(\mu \mathrm{m})$. The color codes of the pixels represent emission fluorescence intensity values in arbitrary units (a. u.). The $D$ and $A$ fluorescence emission regions are marked with green and red boxes, respectively. The radii of the droplets on the spectral images are marked with black dashed lines (---). c. The $I E F_{E T}$ values are plotted against the emission wavelengths for different regions (C, IC, OC and P) for d2, d5 and d20 droplets. d. The maximum $I E F_{E T}$ values (measure of FRET) are plotted for P, OC, IC and $\mathrm{C}$ for $\mathrm{d} 2$, $\mathrm{d} 5$ and $\mathrm{d} 20$. The experiment is carried out 2 independent times with similar results.

\section{Spatiotemporal FRAP shows diffusivity of $\alpha$-Syn molecules are slower at the center compared to}

\section{the periphery of the droplets}

To find more evidence supporting the presence of a solid-like core, we also performed FRAP at different locations (C and OC) inside the same $\alpha$-Syn droplet at different time-points (d2, d5, d7 and d15) (Fig 4a). Furthermore, to provide additional evidence for the formation and maturation of the solid-like core, we have now performed spatiotemporal FRAP with NHS-rhodamine labeled (10\% v/v labeled) $\alpha$-Syn droplets. Briefly, $200 \mu \mathrm{M}$ of $\alpha$-Syn was phase separated in presence of 10\% (w/v) PEG-8000 in $2 \mathrm{ml}$ eppendorf tubes (as mentioned in the manuscript). At regular time intervals (d2, d5, d7 and d15), $5 \mu$ LLPS sample was drop-casted on a clean glass-slide and subjected to FRAP measurements. The droplets were bleached at three regions along their diameter: R1 (center-C), R2- (away from center-OC) and R3- (at the peripheryP) (Fig 4a). Since the d 2 droplets were very small, due to the resolution limit of the confocal microscope, no spatially resolved bleaching could be performed (Fig 4b-c).

At d5, d7 and d15, the droplets become fairly large to perform FRAP at different locations along the diameter without any significantly overlapping bleaching region. Our results showed that at d2 and d5, all the regions (C, OC and P) showed complete fluorescence recovery. However, the kinetics/rate of fluorescence recovery was considerably slower at the center $(\mathrm{C})$ followed by OC and $\mathrm{P}$ regions for $\mathrm{d} 5 \mathrm{drop}-$ lets (Fig 4b-c). The fluorescence images were converted to thermal LUT to probe the difference in the fluorescence recovery at the $\mathrm{C}$ region compared to the OC and $\mathrm{P}$ regions (Figure $\mathbf{4 b - c}$ and Supplementary video 1). At d7, the fluorescence intensity did not recover when the droplets were bleached at the $\mathrm{C}$ region (Fig 4b-c). Strikingly, the OC region of the same droplet showed almost $50 \%$ recovery of fluorescence indicating that the molecules away from the $\mathrm{C}$ region were relatively more diffusive. At the $\mathrm{P}$ region, the recovery was almost $\sim 100 \%$ (Fig 4b-c). Our data clearly points out that at the C region, because of aggregation; the $\alpha$-Syn molecules were restricted compared to the molecules at the periphery of the droplets at d5 and d10 which was very consistent with our FLIM, FTIR, FRET observations. As expected, at $\mathrm{d} 15$, the $\mathrm{C}, \mathrm{OC}$ and $\mathrm{P}$ regions showed minimal recovery of fluorescence indicating propagation of the solid-like core to the surface of the droplet (Fig $4 \mathbf{b}-\mathbf{c}$ ). 
a

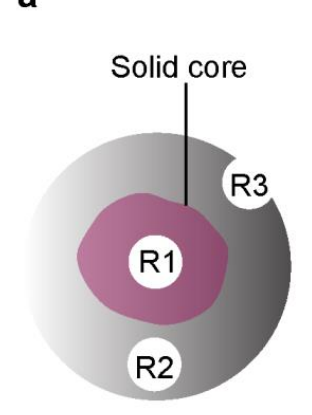

$\mathrm{R} 1=$ Bleaching inside core $(\mathrm{C})$

$\mathrm{R} 2=$ Bleaching outside core $(\mathrm{OC})$

$\mathrm{R} 2=$ Bleaching at the periphery

$(\mathrm{P})$

b

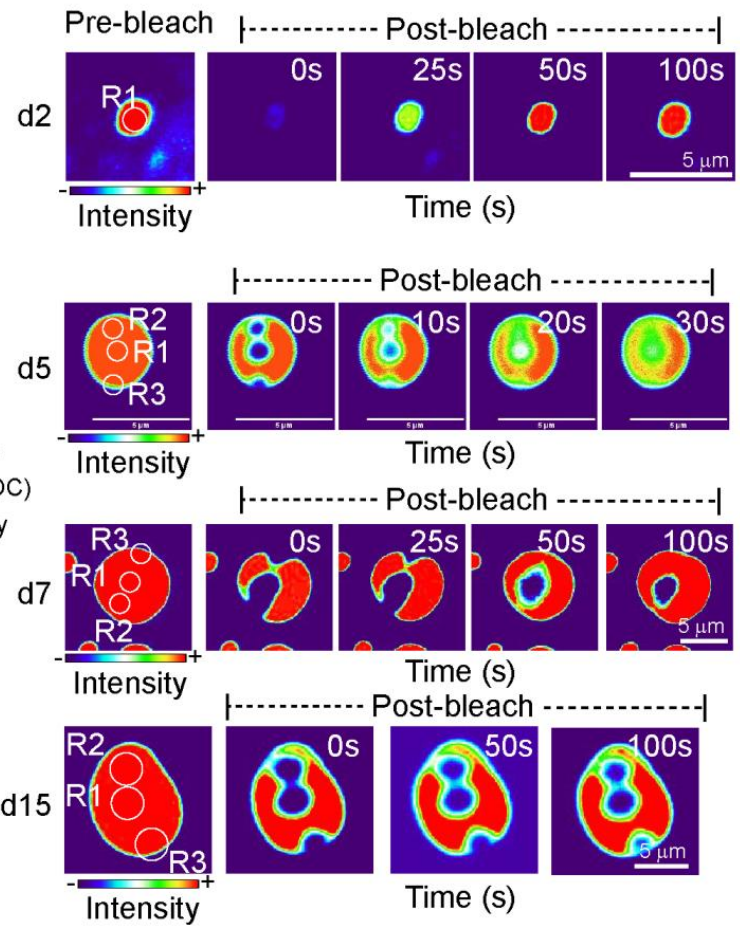

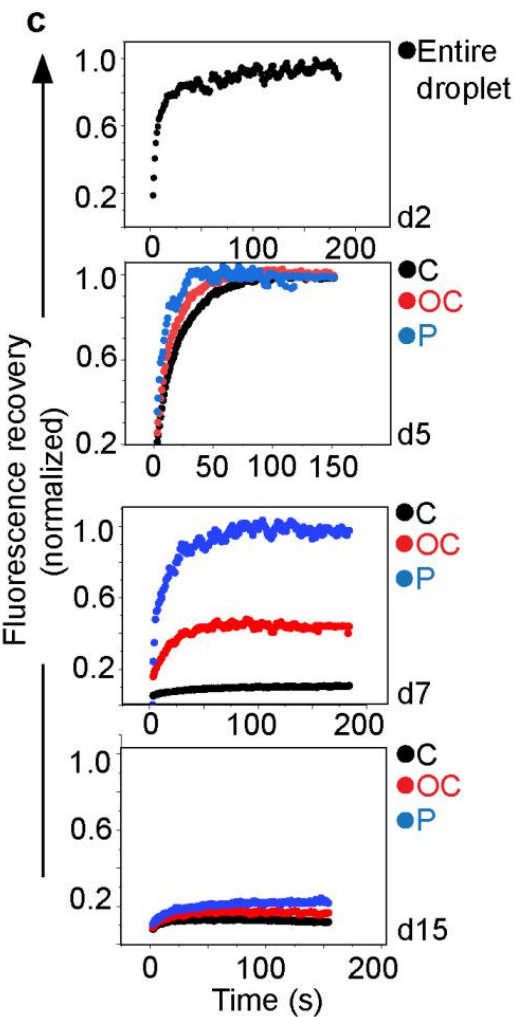

Figure 4: FRAP at different locations of $\alpha$-Syn phase separated droplet during liquid-to-solid transition

a. Schematic showing the bleaching regions chosen for our FRAP study. R1 represents a bleaching region at the center of the droplets where the solid-like core is present. R2 represents a bleaching region away from the core (at the OC region) where the molecules are supposed to be relatively more diffusive. R3 is at the periphery where the molecules are most diffusive. b. Representative time-lapse images of the droplets at d2, d5, d7 and d15 during FRAP are shown. The droplets are bleached at C (R1), OC (R2) and P (R3) regions which are marked with white circles. Notably, $\mathrm{d} 2$ droplets were small and could not be bleached in a spatially resolved manner. The fluorescence recovery is shown in thermal LUT for better visualization of the apparent differences in the rate of the fluorescence recovery for different regions. c. The normalized fluorescence recovery is shown for $\mathrm{C}, \mathrm{OC}$ and $\mathrm{P}$ regions at different time-points (d2-d15). The normalized recovery of the entire droplet is shown for $\mathrm{d} 2$.

\section{Direct observation of solidification at the center of liquid droplets using transmission electron mi- croscopy (TEM) imaging}

To directly confirm the presence of solid-like core at the center of the liquid droplets, we performed timedependent negative stained TEM imaging of $\alpha$-Syn liquid droplets during liquid-to-solid transition (d2d15). The grayscale intensity profiles from TEM images were analyzed using ImageJ (NIH). The detailed description of the analysis is given in the supplementary information.

At the beginning of the droplet formation (d2), TEM imaging showed circular droplet-like structures, which mostly appeared to have amorphous protein content (Fig 5a-b). Image analysis and subsequent grayscale intensity profiles were plotted along the diameter of the droplets, which suggested homogeneous appearance of droplet without any protein rich, electron transparent region inside the d2 droplets (Fig 5c). 
Interestingly, the TEM images of d5 droplets clearly showed formation of a protein rich structure (electron transparent region in negative staining) at the center with a clear ring-like boundary inside the droplets (Fig 5a-b). This could be the solid-like core that formed at the center of the droplets after d5. The signal intensity profiles along the diameter of the droplets showed sharp grayscale intensity drops corresponding to the boundary of the core structure (Fig 5c). The solid-like core structures further grew in size when droplets were allowed to age (Fig 5a-b). Eventually, after d25, amyloid fibrils started emerging from the droplets as evident from our TEM imaging (Fig 5b). Our observation was further evident from the distance between the two grayscale intensity drops in Fig 5c. We further quantified the \% of radius of the solid core based on the electron transparent area at the center of the droplets during time (d5, d10 and d15) using images of multiple droplets (Fig 5c).

We converted the TEM images into angular maps $\left(0-180^{\circ}\right)$ from the periphery to the center to ease the quantification (Fig 5d, Left panel and Supplementary Fig. 10). This operation straightened the images for easy visualization and quantification of the radial average intensity profile of a curved object ${ }^{67}$. The angular maps quantification data indicated that at $\mathrm{d} 5$, the solid-like core covered $\sim 50 \%$ of the radius. At $\mathrm{d} 10$, the core progressed and covered up to $\sim 60 \%$ of the droplet radius and finally, at $\mathrm{d} 15$, the aggregate core covered almost the entire droplet ( $90 \%$ of the radius) (Fig 5d, Right panel).

Our TEM data thus conclusively proves that the initiation and subsequent progression of $\alpha$-Syn aggregation inside the droplets starts at the center and reaches the periphery during liquid-to-solid transition of $\alpha$ Syn LLPS. Additionally, cryo-SEM imaging of d5 droplets also revealed a core-like structure at the center of the $\alpha$-Syn droplets (Supplementary Fig. 11) further supporting our TEM observations. 


\section{a}
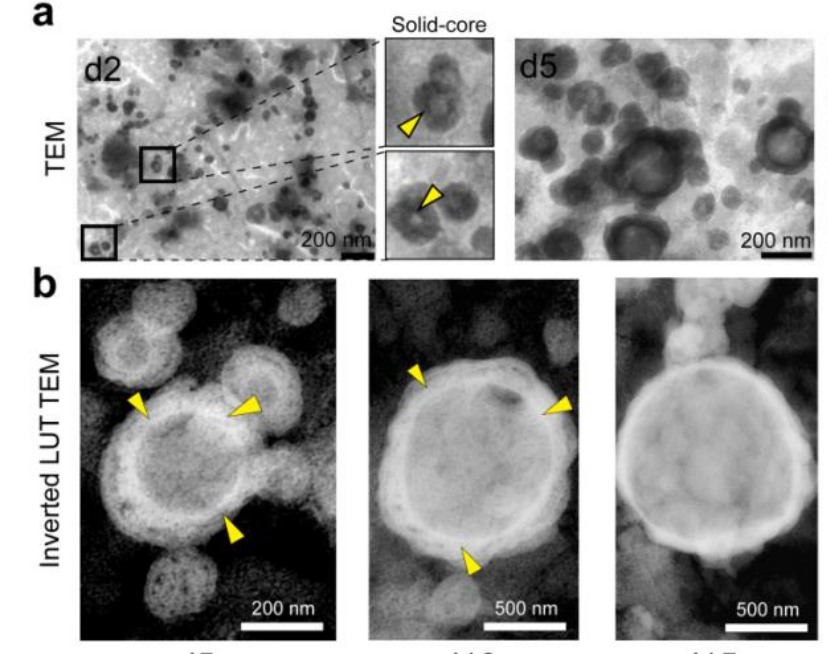

C
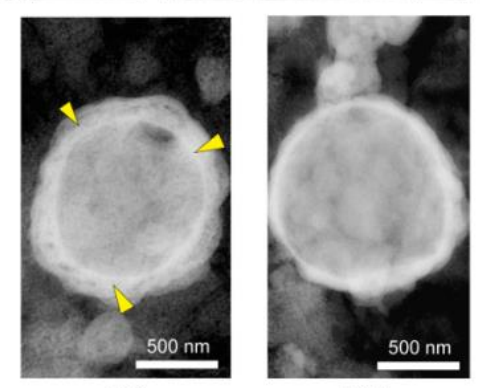

d10

d15

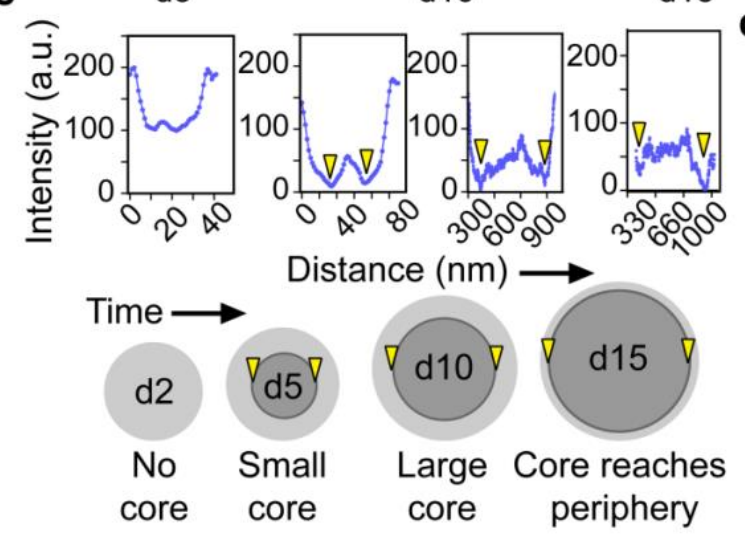

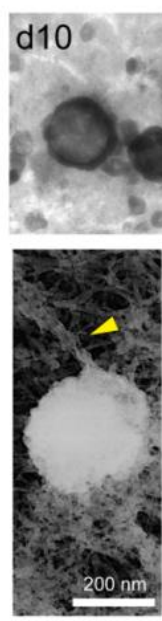

d25
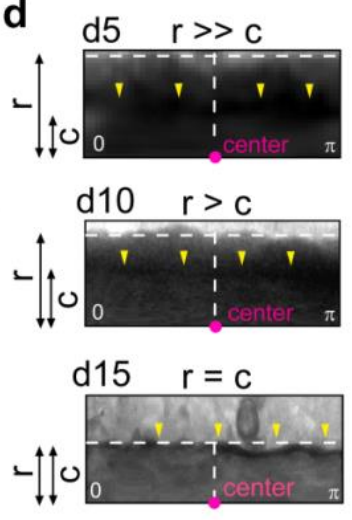
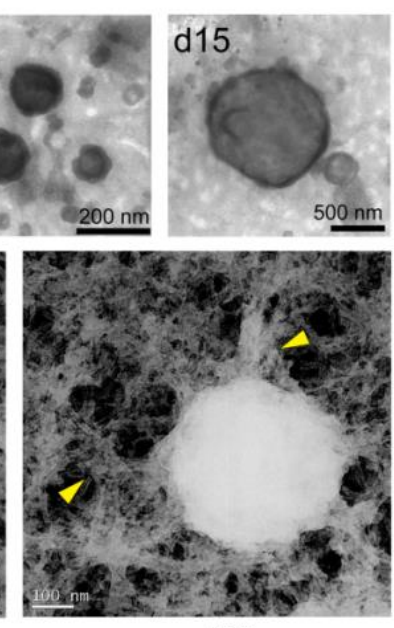

d28

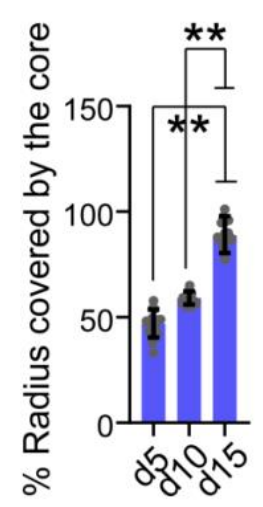

Figure 5: Transmission electron microscope (TEM) images of $\alpha$-Syn liquid droplets during liquid-to-solid transition

a. TEM images of $\alpha$-Syn phase separated droplets at $\mathrm{d} 2$ shows very few droplets which possess the core-like structures (marked with yellow triangular pointers). However, at d5 and d10, the core structure is clearly visible for most droplets. After d15, the core structure is no longer visualized. This could be due to the fact that the core reaches the periphery of the droplet with time. b. Representative TEM images of the droplets at d5, d10 and d15 with inverted lookup table (LUT) clearly indicates the presence of a progressively growing core inside the droplets (marked with yellow triangular pointers). At d25 and d30, fibril-like morphology emerges from the droplets (marked with yellow triangular pointers) indicating amyloid fibril formation starts inside the droplets ${ }^{55}$. c. Upper panel: The pixel intensity is calculated from the cross section (white dashed line) of the droplet at various time-points from d2 to d15. The yellow triangular pointers denote the intensity drops indicating the start and end of the boundary of the solidlike core structure. Lower panel: Schematic representation of the droplets at different time-points (d2-d15) where the $\mathrm{d} 2$ droplet showing the absence of the solid-like core. The core structure appears at $\mathrm{d} 5$ and extends towards the periphery with subsequent incubation. The yellow triangular pointers denote the boundary of the core at each timepoint where the intensity drop is observed. d. Left panel: Angular maps $\left(0 \rightarrow 180^{\circ}\right)$ of the droplets at d5, d10 and $\mathrm{d} 15$ from the center $(\bullet)$ to the periphery (white dashed horizontal line) are generated. The yellow triangular pointers denote the boundary of the core at each time-point. The radius of the entire droplet is denoted as " $r$ " and the radius of the core is denoted as " $\mathrm{c}$ ". Visual observations suggest that at $\mathrm{d} 5, \mathrm{r}>>\mathrm{c}$; at $\mathrm{d} 10, \mathrm{r}>\mathrm{c}$ and at $\mathrm{d} 15, \mathrm{r} \approx \mathrm{c}$. Right panel: The $\%$ radius of the droplet that is covered by the solid core showing significantly higher core at d15 ( 90\%) compared to d10 $(\sim 60 \%)$ and $\mathrm{d} 5(\sim 50 \%)$. The values represent mean \pm S.D. The statistical significance is calculated using two tailed paired Student-t-test with a $95 \%$ confidence interval for $\mathrm{n}=20$ individual droplets. The experiment is performed three times with similar results. 


\section{Fusion of different sized droplets contributing towards droplet growth and liquid-to-solid transition}

Furthermore, we were interested to know how droplet fusion could contribute to the growth and subsequent solidification of $\alpha$-Syn LLPS. We hypothesized that the larger droplets might acquire solid-like core faster than the smaller droplets. Subsequently during fusion, the smaller droplets without the structured core could contribute their contents for further growth of larger droplets (due to difference in their thermodynamic stability). To examine the possible differences in the structured core, we carefully chosen the fusion events of droplets formed by fluorescein-5-maleimide labeled $74 \mathrm{C}-\alpha-\mathrm{Syn}(10 \% \mathrm{v} / \mathrm{v})$ where one droplet was larger compared to the other droplet undergoing fusion at d5 (LD1- larger droplet, LD2smaller droplet). We performed FLIM at selected data-points (p1-p6) along the longitudinal fusion axis, which connects the centers of the droplets (p3 and p6) with the fusion region (p1) (Fig 6a, Left panel). The microscopic observation of the droplets using FLIM showed distinct differences in the $\tau_{\mathrm{m}}$ at different regions of the droplets (Fig 6a, Right panel). Further, the time-resolved fluorescence intensity decay from each of the data-points were collected and fitted with bi-exponential decay function (Fig 6b, Left panel). The decay profiles and corresponding $\tau_{\mathrm{m}}$ data suggested that the central region of both the droplets had lower $\tau_{\mathrm{m}}$ (p6 1.3 ns and p3 $\sim 2 \mathrm{~ns}$ ) while the periphery had higher $\tau_{\mathrm{m}}(\sim 3 \mathrm{~ns})$. Interestingly, the fusion region showed similar $\tau_{\mathrm{m}}$ as the periphery ( $3 \mathrm{~ns}$ ) (Fig 6b, Right panel). In addition, the center of the large droplet showed much less $\tau_{\mathrm{m}}$ compared to the smaller droplet suggesting that the larger droplet possessed a more stable structured core for solidification compared to the smaller droplets. This is further consistent with FTIRM data of two different sized droplets undergoing fusion at d5 (Fig 6c).

The FTIR spectra from the images of two fusing droplets at d5 showed majorly RC conformation for $\mathrm{p} 1$ (at the fusion junction) and p2 (center of smaller droplet). The center of larger sized droplets, p3 however showed $\alpha$-helix conformation (Fig 6d, Left panel and Supplementary table 2). The \% abundance of the secondary structure at different regions is provided in Fig 6d, Right panel and Supplementary table 4. This observation suggests that immature (smaller, thermodynamically unstable) droplets tend to fuse with more stable (bigger) droplets containing a stable solid-like core. 

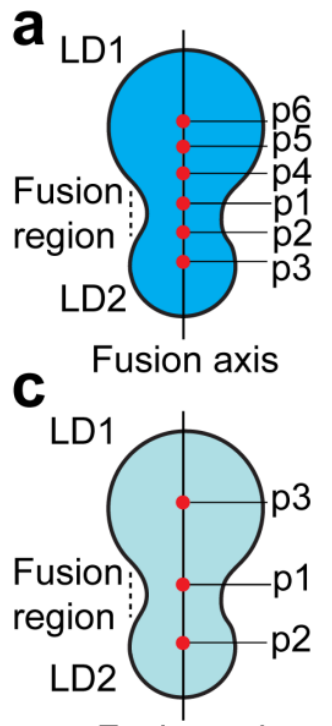

Fusion axis

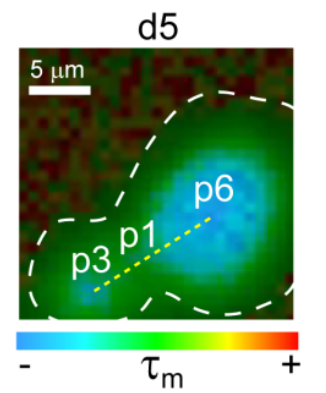

b

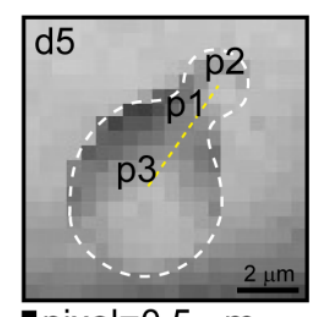

-pixel=0.5 $\mu \mathrm{m}$
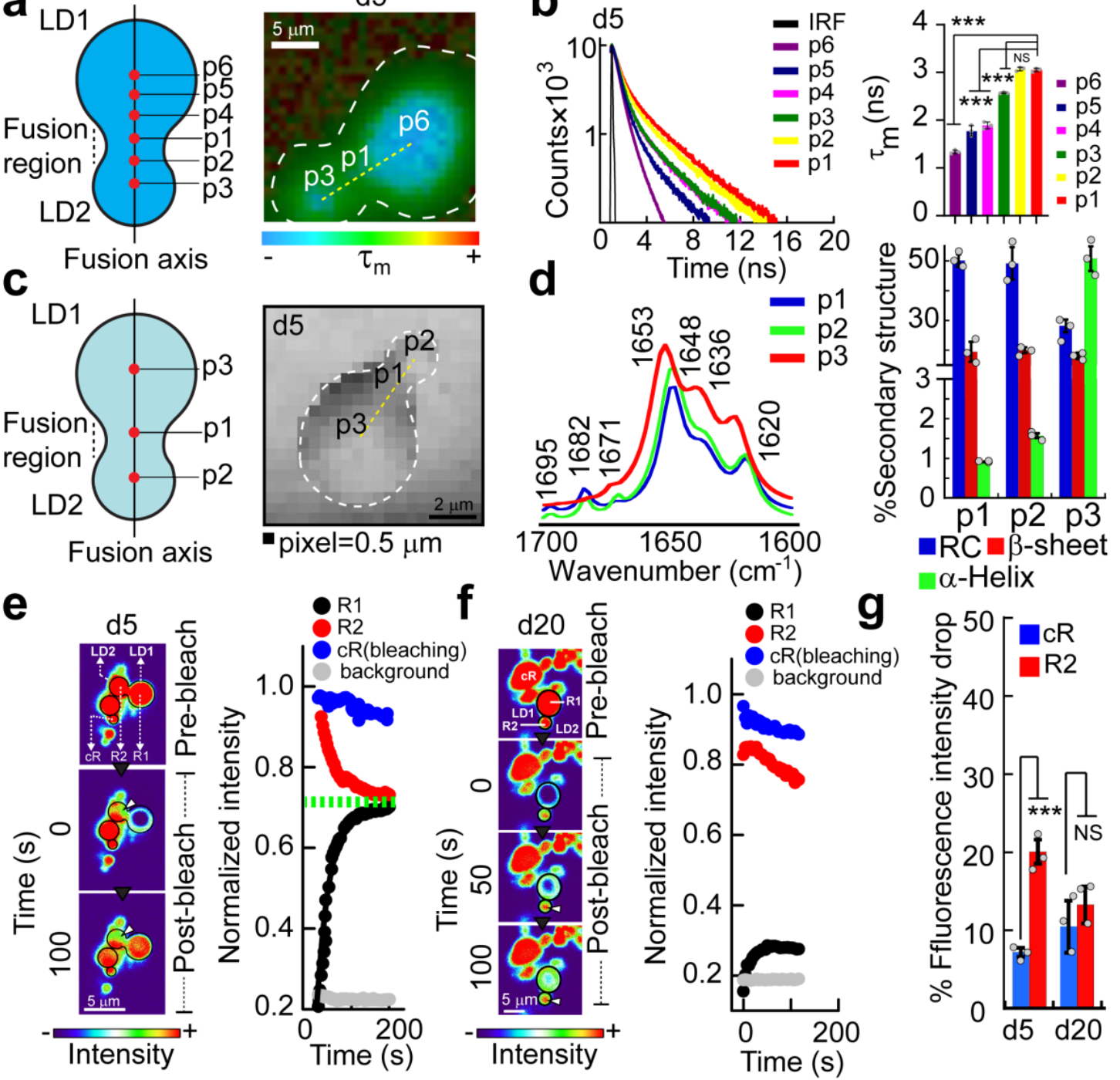

Figure 6: Growth of the larger droplets containing structured core at the center upon fusion with smaller droplets

a. Schematic (left panel) and corresponding original FLIM image (right) showing a fusion event of two heterogeneous sized droplets at $\mathrm{d} 5$. The boundary of the fusion event and longitudinal fusion axis (right panel) is marked with a white dashed curve and yellow dashed line, respectively. The color spectrum represents the range of overall mean lifetime $\left(\tau_{\mathrm{m}}\right)$ (blue is the lowest and red is the highest). b. Left panel: The time resolved fluorescence intensity decay profiles obtained from different regions (from 1 to p6) on the fusing droplets. The black line represents the IRF. Different color codes are assigned to denote different positions from $\mathrm{p} 1$ to $\mathrm{p} 6$. Right panel: The $\tau_{\mathrm{m}}$ for each data point (from $\mathrm{p} 1$ to $\mathrm{p} 6$ ) is plotted. Values represents mean \pm S.D for $\mathrm{n}=3$ independent experiments. The significance is computed using one way ANOVA followed by Student-Newman-Keuls (SNK) post hoc test with a 95\% confidence interval for $\mathrm{n}=3$ independent experiments. c. Schematic (left panel) and single point FTIRM (right panel) image of fusion event between two heterogeneous sized droplets (liquid droplet 1 (LD1) and liquid droplet 2 (LD2)) at d5. The boundary of the fusion event and longitudinal fusion axis (right) is marked with a white dashed curve and yellow dashed line, respectively. d. Left panel: Corresponding FTIR spectra obtained from different regions (p1-p3) of the fusion event at $\mathrm{d} 5$. The respective wavenumber $\left(\mathrm{cm}^{-1}\right)$ for every peak in each spectrum is denoted. Representative spectra are shown. The experiment is carried out three times with similar results. Right panel: The $\%$ abundance of the secondary structures $(\mathrm{RC}, \alpha$-helix and $\beta$-sheet) for each of the data-points ( $\mathrm{p} 1, \mathrm{p} 2$ and p3) is plotted. Values represent mean \pm S.D for $n=3$ independent experiments. e-f. Left panel: Representative images of FRAP by two fusing droplets (LD1 and LD2) at d5 and d20. Liquid droplet 1 (large, LD1) is actively bleached at region ' $\mathrm{R} 1$ ' (diameter of bleaching region $=3 \mu \mathrm{m}$ ). The fluorescence intensity recovery post bleaching 
is acquired from both R1 in LD1 and R2 in LD2. The gradual intensity decrease due to prolonged laser exposure (passive bleaching) is quantified from an independent region of interest (cR) on a different droplet. The images are represented in "thermal" pseudo-color. The color spectrum represents fluorescence intensity (blue, lowest and red, highest). e-f. Right panel: Corresponding post-bleach fluorescence intensities of R1, R2, cR and background. At d5 (e), the post-bleach intensity of R1 gradually increases with time (recovery) while the intensity of R2 gradually decreases. The intensity of R1 and R2 eventually reaches equilibrium (denoted as green dashed line). At d20 (f), the post-bleach intensity of R1 neither recover with time nor the intensity of R2 substantially decreases (only decreases to some extent due to passive laser bleaching). Representative data is shown. The experiment is performed three times with similar results. g. The $\%$ decrease in the fluorescence intensity of cR (due to passive laser bleaching) and R2 (due to molecule transfer to the neighboring fusion partner) are plotted at different time-points (d5 and $\mathrm{d} 20$ ). The data showing the $\%$ fluorescence intensity drop of R2 is significantly higher than passive laser bleaching $(\mathrm{cR})$ at early time-points (d5). The values represent mean \pm S.D. The statistical significance is calculated using two tailed paired Student-t-test with a 95\% confidence interval for $\mathrm{n}=3$ independent experiments.

To probe that smaller droplets without structured core supply the content to larger droplets for their further growth, we performed FRAP of droplets undergoing fusion events at d5. The early stage of droplet fusion events was chosen to ease detection of substantial differences (if any) between two fusing droplets (Fig 6e, Left panel). We used NHS-rhodamine labeled (10\% labeled; v/v) $\alpha$-Syn as passive photobleaching of rhodamine is much less compared to fluorescein ${ }^{68}$. The larger droplet undergoing fusion was actively bleached with laser and its fluorescence recovery was observed. Strikingly, we noticed, as the bleached droplet recovered, the intensity of its unbleached partner (smaller droplet) was substantially decreased. Moreover, the fluorescence intensities of both the droplets eventually reached equilibrium (Fig 6e, Right panel). For example, at $\mathrm{d} 5$, the intensity of the actively bleached, larger droplet was recovered up to $\sim 80 \%$ but simultaneously, the intensity of the unbleached, smaller partner droplet was decreased by $\sim 20 \%$. The passive bleaching effect by the monitoring laser was negligible $(\sim 8 \%)$. This could be because the unbleached droplet was constantly providing the new $\alpha$-Syn molecules to bleached droplet. Overall, the data provides dynamic, real time evidence that inter-droplet transfer of $\alpha$-Syn occurs during fusion events at d5 and the larger droplets containing structured core indeed grow and solidify during time with expense of small droplets after fusion. In contrast, when similar FRAP experiments were performed with neighboring droplets at d20, no substantial fluorescence recovery of the larger droplet was observed. The unbleached, smaller droplet in proximity also did not show any decrease in fluorescence intensity (except due to passive bleaching by the monitoring laser) (Fig 6f-g). Our observations suggest that at later stages of LLPS, the rigidity of the droplet increases in expense of the molecular diffusion, and as a result, the mass transfer between two droplets in proximity is also stopped. Together, the data shows that although $\alpha$-Syn aggregation initiates at the center of the droplet, the monomeric, soluble-like $\alpha$-Syn participates in the molecular exchange during early stages of fusion. 


\section{Discussion}

Previously, many proteins undergoing LLPS showed the phenomenon of liquid-to-solid transition during

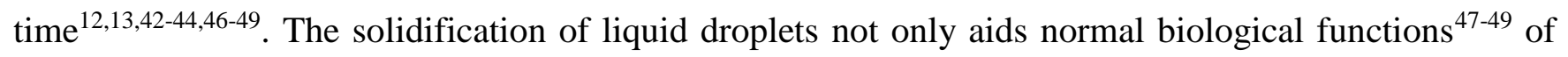
many liquid droplets but also promote toxic protein aggregation associated with various neurological disorders as shown for FUS ${ }^{41,42}, \mathrm{Tau}^{43,44}, \mathrm{TDP}-43^{57}$ and $\alpha-\mathrm{Syn}^{55}$. In the phase-separated droplets undergoing solidification, all molecules might not be in exactly similar microenvironment. Also, it is unlikely that liquid-to-solid transition of protein LLPS is a simple two-state process, where all protein molecules in liquid state are transitioned into solid state at once. Rather, the pathway of liquid-to-solid transition might resemble crystallization process where at first; a nucleus is formed, which gradually recruits molecules for further crystallization" 69 . We asked, "Where exactly does solidification (nucleation event) begin for liquid droplets undergoing liquid-to-solid transition?"

Our experiments on $\alpha$-Syn droplets showed that at the beginning of droplet formation, all $\alpha$-Syn molecules possess similar microenvironment and have monomeric (RC) conformation (Fig 1-2). During maturation of $\alpha$-Syn LLPS, the local microenvironment begins to change at the center (with reduced lifetime of fluorophore attached to $\alpha$-Syn). This change could be associated with $\alpha$-Syn assembly as evident from decrease in fluorescence lifetime and conformational transitions. Previous reports have shown that molecular entanglement during $\alpha$-Syn aggregation results in self-quenching ${ }^{70}$ of the attached fluorescent probe due to close proximity ${ }^{64}$. This leads to a reduced fluorescence lifetime during aggregation and fibril formation. During time, the center of the droplet shows conformational transition into $\alpha$-helix-rich state, an intermediate known for $\alpha$-Syn aggregation, LLPS and gel formation ${ }^{55,65}$. The local microenvironment of $\alpha$-Syn at the center of the droplet further changes (reduced lifetime), which expands and covers the entire droplet with time (Fig 1). FTIRM imaging also shows structural transition of $\alpha$-Syn favorable for aggregation and amyloid formation initiating at the center and then gradually progressing towards the periphery till entire droplet shows $\beta$-sheet-rich amyloid fibril structure (Fig 2). The spatially resolved, position specific measurements, therefore, enables us to identify the solid-like core at the center, which acts as a nucleation site for subsequent solidification/aggregation inside the droplets. Important to note, the kinetics of $\alpha$-Syn LLPS $^{71}$ might influence the observation of the solid-like core. Conditions that triggers immediate LLPS and accelerated liquid-to-solid transition ${ }^{59,60}$ could result in formation and subsequent propagation of the solid-like core within minutes to hours. On the other hand, our method is useful to delineate the spatiotemporal changes with higher resolution because of the slow kinetics of the system.

The probable reason behind solidification starting at the center could be because the environment of $\alpha$ Syn molecules at the surface of the droplet being very different from that of the interior. Each $\alpha$-Syn 
molecule at the interior of the droplet is surrounded by many other $\alpha$-Syn molecules in all directions, resulting in very less amount of net force exerted on these molecules residing deep in the center of a droplet compared to the surface. In contrast, $\alpha$-Syn molecules at the surface of a droplet have asymmetrical environment due to close proximity of the boundary where they experience strong interaction on one side (towards the center) and minimal/no interaction on the other side (outside). Moreover, because the phaseseparated bodies are membraneless, there could also be very fast exchange of the surface $\alpha$-Syn molecules with the surrounding. High surface energy of such $\alpha$-Syn molecules makes them energetically unstable and therefore, prevents stable assembly. Moreover, increased local concentration of $\alpha$-Syn at the center might also trigger self-assembly faster than the other places (such as periphery) of the droplet. This is shown in concentration distribution in PGL3 condensates ${ }^{72}$ and LLPS associated with functions such as heterochromatin assembly ${ }^{49}$ —indicating that the presented phenomenon could be the generic property for many protein condensates undergoing liquid-to-solid transition.

Indeed, our FRET analysis suggests that the intermolecular NAC-domain interaction responsible for amyloid aggregation plays a crucial role in forming the solid-like core, which initiates at the center on d5 and extends towards the surface of the droplet during liquid-to-solid transition (Fig 3). This is further supported by the difference in the \% recovery of fluorescence at different location of the same droplet (Fig 4). Indeed a core-like structure is also observed under TEM at earlier time-points (Fig 5). However, at this point, we cannot exclude the possibility of multiple nucleation (but very small in size) events, which are close to each other that, may occur inside the droplet simultaneously.

During droplet maturation, three distinct processes generally occur: continuous consumption of new $\alpha$ Syn molecules from the solution into the droplet; Ostwald ripening ${ }^{73}$ and droplet fusion ${ }^{74}$. Smaller and thermodynamically unstable droplets tend to fuse with larger, stable droplets to minimize free energy ${ }^{4}$. We hypothesize that during fusion, the transfer of constituent molecules takes place from smaller to larger droplets containing solid-like, aggregate core structure.

Using FLIM and single point FTIRM, we show that indeed, the larger droplets possess a solid-like core (low lifetime and more structured $\alpha$-Syn) at the center, which the smaller droplets lack. This indicates a heterogeneous nature of $\alpha$-Syn LLPS (Fig 6). Smaller, homogeneous droplets fuse with larger droplets where the aggregation process has already been started. This means that at any given time, the droplets, which contain a solid-like core contribute towards the progression of liquid-to-solid transition at the cost of the smaller, soluble droplets, which is consistent with our FRAP study. This phenomenon is however completely hindered at the end of solidification because the aggregation of $\alpha$-Syn reaches the periphery of the droplets preventing further molecular diffusion (Fig 6). This observation is in line with previous 
bioRxiv preprint doi: https://doi.org/10.1101/2021.10.20.465113; this version posted October 21, 2021. The copyright holder for this preprint (which was not certified by peer review) is the author/funder. All rights reserved. No reuse allowed without permission.

reports showing an arrested, supersaturated state of protein droplets where the growth of the droplets is completely stopped ${ }^{6}$.

The present data suggests that solidification/aggregation of $\alpha$-Syn liquid droplets initiates at the center due to facilitated self-assembly and/or conformational transition (Fig 7) in a spatiotemporal manner. The current work might help to understand liquid-to-solid transition and fibril formation by many other proteins containing IDRs, which are associated with neurological disorder as well as functional membraneless organelles formation.

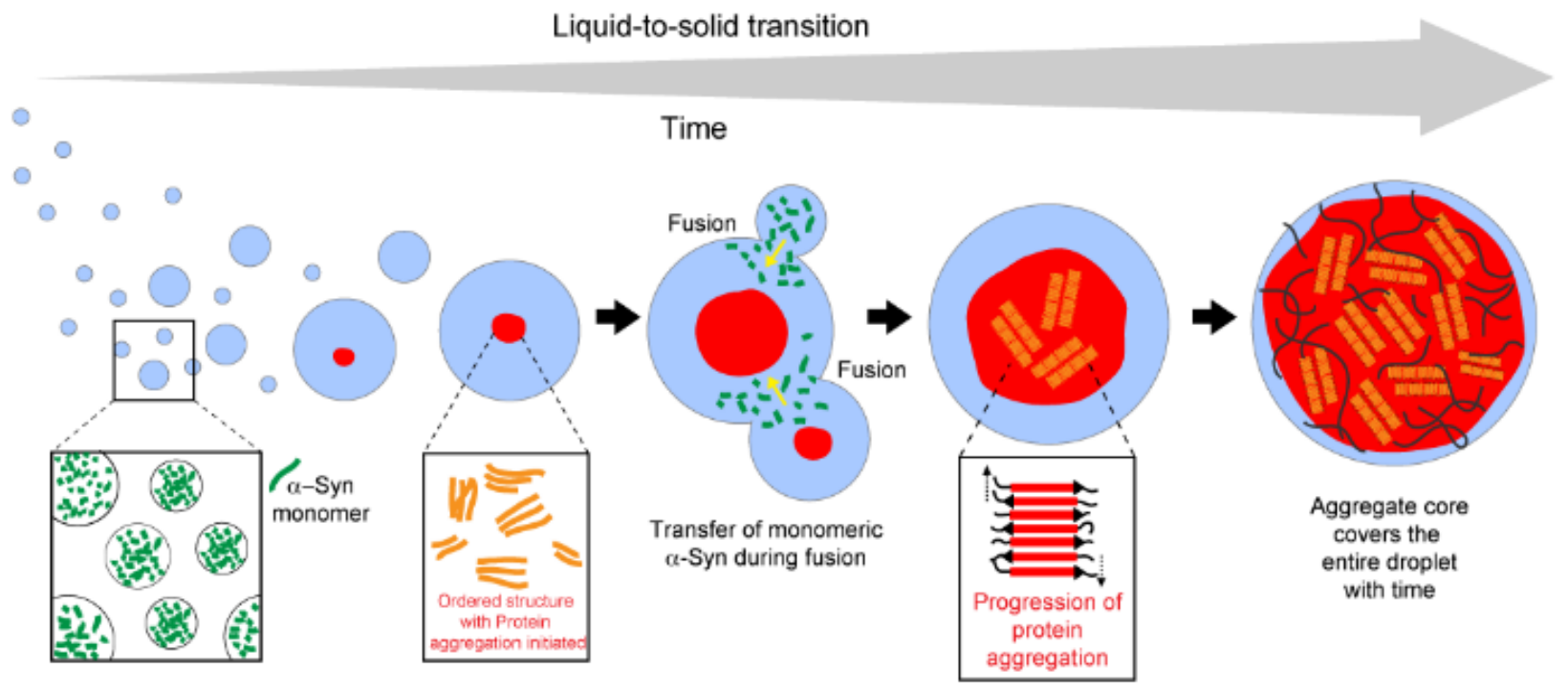

Figure 7: Liquid-liquid phase separation, growth and liquid to solid transition through solid-like core formation at the center of $\alpha$-Syn droplets

The newly formed phase separated $\alpha$-Syn droplets contain monomeric protein. During ageing, a solid-like core appears at the center of $\alpha$-Syn liquid droplets. This core contains ordered structure of $\alpha$-Syn and gradually converts into $\beta$-sheet amyloid with time. The solid-like core expands towards the periphery eventually covering the entire droplet. The growth of the solid-like core (and protein aggregation) is mediated by fusion events. During fusion, smaller, unstable droplets fuse with larger, stable droplets, which already have a structured core. The transfer of monomeric $\alpha$-Syn from the smaller droplet to the larger droplet (denoted by yellow arrows) further helps in the progression of growth and solidification of the larger droplets. 


\section{Supplementary Information}

The Supplementary information file contains materials and methods, Supplementary Fig. 1-11, Supplementary Tables 1-3, Supplementary video legends 1 and 22 references.

\section{AUTHOR INFORMATION}

\section{Corresponding Author}

*Correspondence: Prof. Samir K. Maji, Department of Biosciences and Bioengineering, IIT Bombay, Powai, Mumbai 400 076, India, Tel: + (91-22) 2576-7774, Fax: + (91-22) 2572 3480. Email: samirmaji@iitb.ac.in

\section{Author Contributions}

S.R, D.C, K.P, S.M, J.M, A.S.S, M.P, S. K and R.K performed the experiments. All authors participated in analyzing data. S.R and D.C contributed equally to this work. The study was conceived by S.K.M and designed by S.K.M, G.K, R.P, and A.C. All authors participated in the manuscript writing and approved the manuscript.

\section{Competing interests:}

The authors declare no competing interests.

\section{Data availability statement}

The authors declare that all the data supporting the findings of this study are available within the paper and in supplementary information files. All the data analysis was performed using published tools and packages and has been cited in the paper and supplementary information text.

\section{ACKNOWLEDGMENT}

We acknowledge IIT Bombay central facilities for TEM, FLIM, FTIRM, TCSPC and Confocal microscopy experiments. We thank Jayant B. Udgaonkar for allowing us to utilize FLIM facility. We thank Sreemantee Sen and Lokesh Ranjan for their help in FLIM experiments.

\section{ABBREVIATIONS}

Liquid-liquid phase separation (LLPS); Intrinsically disorder regions (IDRs); Low complexity domains (LCDs); Fluorescence lifetime imaging (FLIM); Single point Fourier transform infra-red microscopy (FTIRM); Förster resonance energy transfer (FRET); Fluorescence recovery after photobleaching (FRAP); Center (C); Inner Circle (IC); Outer circle (OC); Periphery (P) 


\section{REFERENCES}

1 Brangwynne, C. P. et al. Germline P granules are liquid droplets that localize by controlled dissolution/condensation. Science 324, 1729-1732, doi:10.1126/science.1172046 (2009).

2 Banani, S. F. et al. Compositional Control of Phase-Separated Cellular Bodies. Cell 166, 651663 (2016).

3 Shin, Y. \& Brangwynne, C. P. Liquid phase condensation in cell physiology and disease. Science 357, doi:10.1126/science.aaf4382 (2017).

4 Hyman, A. A., Weber, C. A. \& Julicher, F. Liquid-liquid phase separation in biology. Annu Rev Cell Dev Biol 30, 39-58, doi:10.1146/annurev-cellbio-100913-013325 (2014).

5 Boeynaems, S. et al. Protein Phase Separation: A New Phase in Cell Biology. Trends Cell Biol 28, 420-435, doi:10.1016/j.tcb.2018.02.004 (2018).

6 Alberti, S. Phase separation in biology. Curr Biol 27, R1097-R1102, doi:10.1016/j.cub.2017.08.069 (2017).

7 Holehouse, A. S. \& Pappu, R. V. Functional Implications of Intracellular Phase Transitions. Biochemistry 57, 2415-2423, doi:10.1021/acs.biochem.7b01136 (2018).

8 Feric, M. et al. Coexisting Liquid Phases Underlie Nucleolar Subcompartments. Cell 165, 16861697 (2016).

9 Kaiser, T. E., Intine, R. V. \& Dundr, M. De Novo Formation of a Subnuclear Body. Science 322, 1713-1717 (2008).

10 Mitrea, D. M. \& Kriwacki, R. W. Phase separation in biology; functional organization of a higher order. Cell Commun Signal 14, 1, doi:10.1186/s12964-015-0125-7 (2016).

11 Lyon, A. S., Peeples, W. B. \& Rosen, M. K. A framework for understanding the functions of biomolecular condensates across scales. doi:10.1038/s41580-020-00303-z (2020).

12 Molliex, A. et al. Phase separation by low complexity domains promotes stress granule assembly and drives pathological fibrillization. Cell 163, 123-133, doi:10.1016/j.cell.2015.09.015 (2015).

13 Lin, Y., Protter, D. S., Rosen, M. K. \& Parker, R. Formation and Maturation of Phase-Separated Liquid Droplets by RNA-Binding Proteins. Mol Cell 60, 208-219, doi:10.1016/j.molcel.2015.08.018 (2015).

14 Riback, J. A. et al. Stress-Triggered Phase Separation Is an Adaptive, Evolutionarily Tuned Response. Cell 168, 1028-1040 e1019, doi:10.1016/j.cell.2017.02.027 (2017).

15 Larson, A. G. \& Narlikar, G. J. The Role of Phase Separation in Heterochromatin Formation, Function, and Regulation. Biochemistry 57, 2540-2548, doi:10.1021/acs.biochem.8b00401 (2018).

16 Narlikar, G. J. Phase-separation in chromatin organization. Journal of Biosciences 45, 5, doi:10.1007/s12038-019-9978-z (2020).

17 Gibson, B. A. et al. Organization of Chromatin by Intrinsic and Regulated Phase Separation. Cell 179, 470-484.e421, doi:10.1016/j.cell.2019.08.037 (2019).

18 Peeples, W. \& Rosen, M. K. Phase Separation Can Increase Enzyme Activity by Concentration and Molecular Organization. bioRxiv, 2020.2009.2015.299115, doi:10.1101/2020.09.15.299115 (2020).

19 Narayanaswamy, R. et al. Widespread reorganization of metabolic enzymes into reversible assemblies upon nutrient starvation. Proceedings of the National Academy of Sciences 106, 10147-10152, doi:10.1073/pnas.0812771106 (2009).

20 Dzuricky, M., Rogers, B. A., Shahid, A., Cremer, P. S. \& Chilkoti, A. De novo engineering of intracellular condensates using artificial disordered proteins. Nature Chemistry 12, 814-825, doi:10.1038/s41557-020-0511-7 (2020).

21 Ray, S. \& Maji, S. K. Predictable phase-separated proteins. Nature Chemistry 12, 787-789, doi:10.1038/s41557-020-0532-2 (2020). 
22 Banani, S. F., Lee, H. O., Hyman, A. A. \& Rosen, M. K. Biomolecular condensates: organizers of cellular biochemistry. Nature Reviews Molecular Cell Biology 18, 285-298, doi:10.1038/nrm.2017.7 (2017).

23 Pappu, R. V. Phase Separation-A Physical Mechanism for Organizing Information and Biochemical Reactions. Developmental Cell 55, 1-3, doi:https://doi.org/10.1016/j.devcel.2020.09.023 (2020).

24 Boija, A. et al. Transcription Factors Activate Genes through the Phase-Separation Capacity of Their Activation Domains. Cell 175, 1842-1855.e1816, doi:https://doi.org/10.1016/j.cell.2018.10.042 (2018).

25 Klein, I. A. et al. Partitioning of cancer therapeutics in nuclear condensates. Science 368, 13861392, doi:10.1126/science.aaz4427 (2020).

26 Chong, P. A. \& Forman-Kay, J. D. Liquid-liquid phase separation in cellular signaling systems. Curr Opin Struct Biol 41, 180-186, doi:10.1016/j.sbi.2016.08.001 (2016).

$27 \mathrm{Su}, \mathrm{X}$. et al. Phase separation of signaling molecules promotes T cell receptor signal transduction. Science 352, 595-599, doi:10.1126/science.aad9964 (2016).

28 Banjade, S. \& Rosen, M. K. Phase transitions of multivalent proteins can promote clustering of membrane receptors. Elife 3, doi:10.7554/eLife.04123 (2014).

29 Li, P. et al. Phase transitions in the assembly of multivalent signalling proteins. Nature 483, 336340, doi:10.1038/nature10879 (2012).

30 Alshareedah, I., Moosa, M. M., Raju, M., Potoyan, D. A. \& Banerjee, P. R. Phase transition of RNA-protein complexes into ordered hollow condensates. Proceedings of the National Academy of Sciences 117, 15650-15658, doi:10.1073/pnas.1922365117 (2020).

31 Brangwynne, Clifford P., Tompa, P. \& Pappu, Rohit V. Polymer physics of intracellular phase transitions. Nature Physics 11, 899-904, doi:10.1038/nphys3532 (2015).

32 Wei, M. T. et al. Phase behaviour of disordered proteins underlying low density and high permeability of liquid organelles. Nat Chem 9, 1118-1125, doi:10.1038/nchem.2803 (2017).

33 Riback, J. A. et al. Composition-dependent thermodynamics of intracellular phase separation. Nature 581, 209-214, doi:10.1038/s41586-020-2256-2 (2020).

34 Hughes, M. P. et al. Atomic structures of low-complexity protein segments reveal kinked beta sheets that assemble networks. Science 359, 698-701, doi:10.1126/science.aan6398 (2018).

35 Pak, C. W. et al. Sequence Determinants of Intracellular Phase Separation by Complex Coacervation of a Disordered Protein. Mol Cell 63, 72-85, doi:10.1016/j.molcel.2016.05.042 (2016).

36 Maharana, S. et al. RNA buffers the phase separation behavior of prion-like RNA binding proteins. Science 360, 918-921, doi:10.1126/science.aar7366 (2018).

37 Wang, J. et al. A Molecular Grammar Governing the Driving Forces for Phase Separation of Prion-like RNA Binding Proteins. Cell 174, 688-699.e616, doi:10.1016/j.cell.2018.06.006 (2018).

38 Burke, K. A., Janke, A. M., Rhine, C. L. \& Fawzi, N. L. Residue-by-Residue View of In Vitro FUS Granules that Bind the C-Terminal Domain of RNA Polymerase II. Mol Cell 60, 231-241, doi:10.1016/j.molcel.2015.09.006 (2015).

39 Nott, T. J. et al. Phase transition of a disordered nuage protein generates environmentally responsive membraneless organelles. Mol Cell 57, 936-947, doi:10.1016/j.molcel.2015.01.013 (2015).

40 Zhang, H. et al. RNA Controls PolyQ Protein Phase Transitions. Mol Cell 60, 220-230, doi:10.1016/j.molcel.2015.09.017 (2015).

41 Murakami, T. et al. ALS/FTD Mutation-Induced Phase Transition of FUS Liquid Droplets and Reversible Hydrogels into Irreversible Hydrogels Impairs RNP Granule Function. Neuron 88, 678-690, doi:10.1016/j.neuron.2015.10.030 (2015). 
42 Patel, A. et al. A Liquid-to-Solid Phase Transition of the ALS Protein FUS Accelerated by Disease Mutation. Cell 162, 1066-1077, doi:10.1016/j.cell.2015.07.047 (2015).

43 Wegmann, S. et al. Tau protein liquid-liquid phase separation can initiate tau aggregation. EMBO J 37, doi:10.15252/embj.201798049 (2018).

44 Ambadipudi, S., Biernat, J., Riedel, D., Mandelkow, E. \& Zweckstetter, M. Liquid-liquid phase separation of the microtubule-binding repeats of the Alzheimer-related protein Tau. Nat Commun 8, 275, doi:10.1038/s41467-017-00480-0 (2017).

45 Conicella, A. E., Zerze, G. H., Mittal, J. \& Fawzi, N. L. ALS Mutations Disrupt Phase Separation Mediated by alpha-Helical Structure in the TDP-43 Low-Complexity C-Terminal Domain. Structure 24, 1537-1549, doi:10.1016/j.str.2016.07.007 (2016).

46 Pytowski, L., Lee, C. F., Foley, A. C., Vaux, D. J. \& Jean, L. Liquid-liquid phase separation of type II diabetes-associated IAPP initiates hydrogelation and aggregation. Proceedings of the National Academy of Sciences 117, 12050-12061, doi:10.1073/pnas.1916716117 (2020).

47 Boke, E. et al. Amyloid-like Self-Assembly of a Cellular Compartment. Cell 166, 637-650, doi:10.1016/j.cell.2016.06.051 (2016).

48 Kroschwald, S. et al. Promiscuous interactions and protein disaggregases determine the material state of stress-inducible RNP granules. Elife 4, e06807, doi:10.7554/eLife.06807 (2015).

49 Falk, M. et al. Heterochromatin drives compartmentalization of inverted and conventional nuclei. Nature 570, 395-399, doi:10.1038/s41586-019-1275-3 (2019).

50 Mathieu, C., Pappu, R. V. \& Taylor, J. P. Beyond aggregation: Pathological phase transitions in neurodegenerative disease. Science 370, 56-60, doi:10.1126/science.abb8032 (2020).

51 Aguzzi, A. \& Altmeyer, M. Phase Separation: Linking Cellular Compartmentalization to Disease. Trends Cell Biol 26, 547-558, doi:10.1016/j.tcb.2016.03.004 (2016).

52 Alberti, S. \& Dormann, D. Liquid-Liquid Phase Separation in Disease. Annual Review of Genetics 53, 171-194, doi:10.1146/annurev-genet-112618-043527 (2019).

53 Zbinden, A., Pérez-Berlanga, M., De Rossi, P. \& Polymenidou, M. Phase Separation and Neurodegenerative Diseases: A Disturbance in the Force. Developmental cell 55, 45-68, doi:10.1016/j.devcel.2020.09.014 (2020).

54 Munder, M. C. et al. A pH-driven transition of the cytoplasm from a fluid- to a solid-like state promotes entry into dormancy. Elife 5, doi:10.7554/eLife.09347 (2016).

55 Ray, S. et al. $\alpha$-Synuclein aggregation nucleates through liquid-liquid phase separation. Nature Chemistry 12, 705-716, doi:10.1038/s41557-020-0465-9 (2020).

56 Boyko, S., Surewicz, K. \& Surewicz, W. K. Regulatory mechanisms of tau protein fibrillation under the conditions of liquid-liquid phase separation. Proceedings of the National Academy of Sciences, 202012460, doi:10.1073/pnas.2012460117 (2020).

57 Watanabe, S. et al. Aggresome formation and liquid-liquid phase separation independently induce cytoplasmic aggregation of TAR DNA-binding protein 43. Cell Death \& Disease 11, 909, doi:10.1038/s41419-020-03116-2 (2020).

58 Hardenberg, M., Horvath, A., Ambrus, V., Fuxreiter, M. \& Vendruscolo, M. Widespread occurrence of the droplet state of proteins in the human proteome. Proceedings of the National Academy of Sciences 117, 33254-33262, doi:10.1073/pnas.2007670117 (2020).

59 Hardenberg, M. C. et al. Observation of an $\alpha$-synuclein liquid droplet state and its maturation into Lewy body-like assemblies. Journal of Molecular Cell Biology, doi:10.1093/jmcb/mjaa075 (2021).

60 Sawner, A. S. et al. Modulating a-Synuclein Liquid-Liquid Phase Separation. Biochemistry, doi:10.1021/acs.biochem.1c00434 (2021).

61 Lakowicz, J. R. Principles of fluorescence spectroscopy. (2010).

62 Berezin, M. Y. \& Achilefu, S. Fluorescence Lifetime Measurements and Biological Imaging. Chemical Reviews 110, 2641-2684, doi:10.1021/cr900343z (2010). 
63 Giasson, B. I., Murray, I. V., Trojanowski, J. Q. \& Lee, V. M. A hydrophobic stretch of 12 amino acid residues in the middle of alpha-synuclein is essential for filament assembly. $J$ Biol Chem 276, 2380-2386, doi:10.1074/jbc.M008919200 (2001).

64 Laine, R. F. \& Sinnige, T. Fast Fluorescence Lifetime Imaging Reveals the Aggregation Processes of $\alpha$-Synuclein and Polyglutamine in Aging Caenorhabditis elegans. 14, 1628-1636, doi:10.1021/acschembio.9b00354 (2019).

65 Ghosh, D. et al. Structure based aggregation studies reveal the presence of helix-rich intermediate during alpha-Synuclein aggregation. Scientific Reports 5 (2015).

66 Murakami, K. et al. Observation of liquid-liquid phase separation of ataxin-3 and quantitative evaluation of its concentration in a single droplet using Raman microscopy. Chemical Science 12, 7411-7418, doi:10.1039/D0SC06095J (2021).

67 Ianiro, A. et al. Liquid-liquid phase separation during amphiphilic self-assembly. Nature Chemistry 11, 320-328, doi:10.1038/s41557-019-0210-4 (2019).

68 Widengren, J. \& Rigler, R. Mechanisms of photobleaching investigated by fluorescence correlation spectroscopy. Bioimaging 4, 149-157, doi:https://doi.org/10.1002/13616374(199609)4:3<149::AID-BIO5>3.0.CO;2-D (1996).

69 Durbin, S. D. \& Feher, G. PROTEIN CRYSTALLIZATION. Annual Review of Physical Chemistry 47, 171-204, doi:10.1146/annurev.physchem.47.1.171 (1996).

70 Chen, R. F. \& Knutson, J. R. Mechanism of fluorescence concentration quenching of carboxyfluorescein in liposomes: Energy transfer to nonfluorescent dimers. Analytical Biochemistry 172, 61-77, doi:https://doi.org/10.1016/0003-2697(88)90412-5 (1988).

71 Martin, E. W. et al. A multi-step nucleation process determines the kinetics of prion-like domain phase separation. Nature Communications 12, 4513, doi:10.1038/s41467-021-24727-z (2021).

72 McCall, P. M. et al. Quantitative phase microscopy enables precise and efficient determination of biomolecular condensate composition. bioRxiv, 2020.2010.2025.352823, doi:10.1101/2020.10.25.352823 (2020).

73 Voorhees, P. W. Ostwald Ripening of Two-Phase Mixtures. Annual Review of Materials Science 22, 197-215, doi:10.1146/annurev.ms.22.080192.001213 (1992).

74 Berry, J., Weber, S. C., Vaidya, N., Haataja, M. \& Brangwynne, C. P. RNA transcription modulates phase transition-driven nuclear body assembly. Proc Natl Acad Sci US A 112, E5237-5245, doi:10.1073/pnas.1509317112 (2015). 


\section{Supplementary Information}

\section{Spatiotemporal solidification of $\alpha$-synuclein inside the liquid droplets}

Soumik Ray ${ }^{1, \dagger}$, Debdeep Chatterjee ${ }^{1, \dagger}$, Semanti Mukherjee ${ }^{1}$, Komal Patel ${ }^{1}$, Jaladhar Mahato $^{2}$, Sangram Kadam ${ }^{1}$, Rakesh Krishnan ${ }^{1}$, Ajay Singh Sawner ${ }^{1}$, Manisha Poudyal ${ }^{1}$, G Krishnamoorthy $^{3}$, Arindam Chowdhury ${ }^{2}$, Ranjith Padinhateeri ${ }^{1}$ and Samir K. Maji ${ }^{*}$

${ }^{1}$ Department of Biosciences and Bioengineering, IIT Bombay, Powai, Mumbai-400076, India

${ }^{2}$ Department of Chemistry, IIT Bombay, Powai, Mumbai 400076, India

${ }^{3}$ Department of Biotechnology, Anna University, Chennai 600025, India †Contributed equally

*Correspondence: Prof. Samir K. Maji, Department of Biosciences and Bioengineering, IIT Bombay, Powai, Mumbai 400 076, India, Tel: + (91-22) 2576-7774, Fax: + (91-22) 25723480. Email: samirmaji@iitb.ac.in 


\section{Supplementary materials and methods:}

\section{Reagents and chemicals}

All the reagents and chemicals used in the following experiments were purchased from ThermoFisher Scientific (USA), Sigma (USA), and HiMedia (India) unless otherwise mentioned. Fluorescein-5-maleimide, rhodamine-C2-maleimide and NHS-rhodamine dyes were purchased from ThermoFisher Scientific (USA).

\section{Expression and purification of recombinant $\alpha-S y n$}

Protein expression and purification was performed using previously established protocol ${ }^{1-3}$. Briefly, E.coli BL21-DE3 cells were transformed with pRK172 plasmids encoding for WT and $74 \mathrm{C}-\alpha$-Syn under inducible lac-promoter. The expression of the protein(s) was induced by the addition of $1 \mathrm{mM}$ isopropyl- $\beta$-D-thiogalactoside (IPTG) to the bacterial culture. After that, the cells were harvested by centrifuging the culture at $4000 \mathrm{rpm}$ for $30 \mathrm{~min}$ at $4{ }^{\circ} \mathrm{C}$. The cell pellet is dissolved in appropriate volume of $\alpha$-Syn buffer $-50 \mathrm{mM}$ Tris, $\mathrm{pH}$ 8.0, $10 \mathrm{mM}$ EDTA, $150 \mathrm{mM}$ $\mathrm{NaCl}$ along with protease inhibitor cocktail (Roche) and subsequently lysed with the help of a probe sonicator (Sonics and Materials Inc. USA) at $4{ }^{\circ} \mathrm{C}$. For $74 \mathrm{C}-\alpha-$ Syn, sonication and further steps were performed under a reducing environment in the presence of $1 \mathrm{mM}$ dithiothreitol (DTT) to prevent intermolecular di-sulfide linkages. Next, the cell lysate was subjected to heat denaturation on a boiling water bath for $20 \mathrm{~min}$ and centrifuged at $9000 \mathrm{rpm}$ for $30 \mathrm{~min}$ at $4{ }^{\circ} \mathrm{C}$. $10 \%(\mathrm{w} / \mathrm{v})$ streptomycin sulphate and glacial acetic acid was added to the supernatant to precipitate the nucleic acid (DNA) contaminants. The solution is then centrifuged at $9000 \mathrm{rpm}$ for $30 \mathrm{~min}$ at $4{ }^{\circ} \mathrm{C}$ to pellet the DNA and the supernatant is collected. Equal volume of ice-cold, saturated ammonium sulphate and ultrapure (99\%), molecular grade ethanol was added to the supernatant and the solution was kept at $4{ }^{\circ} \mathrm{C}$ for $\sim 12 \mathrm{~h}$ to precipitate the protein. The solution is centrifuged at $10000 \mathrm{rpm}$ for $40 \mathrm{~min}$ at $4{ }^{\circ} \mathrm{C}$ to pellet the protein. The protein pellet was subsequently washed with $100 \mathrm{mM}$ ammonium acetate for 3 to 4 times and precipitated using equal volume of ultrapure ethanol. The protein was finally dissolved in minimum volume of $100 \mathrm{mM}$ ammonium acetate and lyophilized. The lyophilized protein powder was kept at $-80^{\circ} \mathrm{C}$ for future use. Before and during experiments, the protein quality is checked using SDS-PAGE and MALDI-TOF mass spectrometry (Supplementary Fig. 1-2).

\section{Preparation of aggregate free low molecular weight (LMW) $\alpha-S y n$}

Low molecular weight (LMW) $\alpha$-Syn was prepared using a standard protocol ${ }^{3-5}$. Briefly, lyophilized protein was dissolved in $20 \mathrm{mM}$ phosphate buffer ( $\mathrm{pH} 7.4$ ), $0.05 \%$ sodium azide, and solubilized by adding few drops of $0.2 \mathrm{~N}(\mathrm{NaOH})$. The $\mathrm{pH}$ of the protein solution was adjusted to 7.4 with $2 \mathrm{M} \mathrm{HCl}$ with the help of a micro-pH meter probe (Mettler Toledo, USA). The solution was centrifuged at $12000 \mathrm{rpm}$ for $30 \mathrm{~min}$ at $4{ }^{\circ} \mathrm{C}$ to remove any insoluble impurities. The supernatant was collected and dialyzed against $20 \mathrm{mM}$ phosphate buffer ( $\mathrm{pH} 7.4$ ), 0.05\% sodium azide, for $\sim 12 \mathrm{~h}$ using $10 \mathrm{kDa}$ cut off membranes (Sigma, USA). The dialyzed protein solution 
was then passed through a $100 \mathrm{kDa}$ pre-washed cut-off filter (Merck Millipore, USA) to remove higher order structures (if any). The flow-through contained mostly monomeric $\alpha$-Syn and is termed as the low molecular weight (LMW) fraction. The concentration of the LMW was determined by measuring the ultraviolet (UV) absorbance at $280 \mathrm{~nm}$. The molar extinction coefficient $(\varepsilon)$ of both WT and 74C- $\alpha$-Syn used for determining concentration was of $\left(5960 \mathrm{M}^{-1}\right.$ $\left.\mathrm{cm}^{-1}\right)^{6}$.

\section{Fluorescence labeling of $\alpha-S y n$}

$74 \mathrm{C}-\alpha$-Syn was labeled with fluorescein-5-maleimide at the $74^{\text {th }}$ cysteine residue (at the nonamyloid $\beta$ component (NAC) region) for fluorescence lifetime imaging (FLIM) experiments. 74C$\alpha$-Syn was separately labeled with both fluorescein-5-maleimide and rhodamine-C2-maleimide at the $74^{\text {th }}$ cysteine residue for spectrally (and spatially) resolved Forster resonance energy transfer (FRET) experiments. WT $\alpha$-Syn was labeled with NHS-rhodamine for fluorescence recovery after photobleaching (FRAP) experiments. All the labeling was performed as per the manufacturer's (ThermoFisher Scientific, USA) instructions. Briefly, $500 \mu \mathrm{M}$ of LMW $\alpha$-Syn (WT and 74C) was mixed with 5 molar excess fluorescent dyes (dissolved in dimethyl sulfoxide (DMSO)). The solution was gently mixed at $25{ }^{\circ} \mathrm{C}$ for $2-3 \mathrm{~h}$. Excess or unbound dye was removed by dialysis against $20 \mathrm{~mm}$ phosphate buffer ( $\mathrm{pH} 7.4$ ) at $4^{0} \mathrm{C}$ for $\sim 48$ hours, with regular exchange of the buffer at every $5 \mathrm{~h}$ interval. The labeled protein concentration and the extent of labeling were measured using a UV spectrophotometer according to the formula provided by the manufacturer. For further experiments, mixture of $10 \% \mathrm{v} / \mathrm{v}$ labeled and $90 \% \mathrm{v} / \mathrm{v}$ unlabeled $\alpha$-Syn is used to minimize the effect of labeling on phase separation ${ }^{3}$. Our previous study showed that the presence of $10 \%$ labeled protein does not affect the structure and aggregation property of WT $\alpha$-Syn protein ${ }^{3}$.

\section{In vitro liquid-liquid phase separation (LLPS) of $\alpha$-Syn}

LLPS study was performed according to our previously established protocol ${ }^{3}$. Briefly, LMW $\alpha-$ Syn solution containing $200 \mu \mathrm{M} \alpha$-Syn and 10\% (w/v) PEG-8000 in $20 \mathrm{mM}$ sodium phosphate buffer (pH 7.4), $0.05 \%$ sodium azide, was prepared and used for LLPS. Similar protocol was also used for fluorescent-labeled protein as $\alpha$-Syn shows identical phase behavior irrespective of fluorescent labeling ${ }^{3}$. The LLPS solution (10-15 $\mu$ l) was then spotted onto a clean glass slide and sandwiched with an $18 \mathrm{~mm}$ coverslip (Blue Star, India). The coverslip was then sealed with commercially available nail-polish. The slides were kept in a moist chamber to prevent drying of the LLPS solution and incubated at $37{ }^{\circ} \mathrm{C}$ to initiate LLPS. At regular intervals, the slides were taken out and observed under a DMi8 microscope (Leica Microsystems, Germany) at 63X magnification under differential interference contrast (DIC) mode to check for the formation of liquid droplets. In parallel, identical LLPS samples were also incubated in $2 \mathrm{ml}$ eppendorf tubes and at regular intervals, 4-5 $\mu \mathrm{l}$ of sample was drop-casted on a clean glass slide and visualized under 63X magnification under differential interference contrast (DIC) mode to check for the formation of liquid droplets. The details of sample preparation and microscopic observation for fluorescence based studies (FLIM, FRET, FRAP) and Fourier transform infrared microscopy 
(FTIRM) are given in the respective sections. To check for possible drying of samples, $20 \mu 1$ of the LLPS samples $(200 \mu \mathrm{M} \alpha$-Syn $+10 \%$ (w/v) PEG-8000) were drop-casted onto $6 \mathrm{~mm}$ depression slides and incubated in a moist chamber at $37{ }^{\circ} \mathrm{C}$. The moisture was replenished every $24 \mathrm{~h}$. After $\mathrm{d} 20$, the coverslips from the slides are lifted and $5 \mu 1$ of $\alpha$-Syn LLPS sample is pipetted and subsequently diluted 10 times. The samples were heated at $\sim 90{ }^{\circ} \mathrm{C}$ for $20 \mathrm{~min}$ to denature the higher order structures and fibrils. From the diluted solutions, the concentration $(280 \mathrm{~nm}$ absorbance) of $\alpha$-Syn was measured using UV-spectroscopy in the range of 240-340 nm (Supplementary Fig 4).

\section{Design principle for mapping $\alpha$-Syn liquid droplets under microscope}

The $\alpha$-Syn liquid droplets are spherical in nature. However, microscopy based imaging techniques focus primarily on one-plane along the Z-axis (depth) at a time. This resulted in projection of a spherical droplet as a circular structure during image acquisition. For our microscopy based FLIM and FTIRM experiments; at first, the droplets were imaged at their central plane along the Z-axis, as it is expected to give sufficient information in two dimensions (X-Y plane). The central plane of the droplet was divided into three concentric circles. The radius of the inner most circle (inner circle; IC) was taken as " $r$ " and the radii of the next two circles (outer circle: OC and peripheral circle: P) were taken as multiples of " $r$ " ( $2 r$ and $3 r$, respectively). The data-points were chosen at the center $(\mathrm{C})$ of the droplet and on the circumference of each concentric circle. From a single droplet, the number of data-points acquired at C, IC, OC and P were 1, 6, 12 and 4, respectively; for fluorescence lifetime imaging (FLIM). On the other hand, the number of data-points acquired at C, IC, OC and P were 1, 6, 6 and 4, respectively; for Fourier transform infra-red microscopy (FTIRM) studies. To qualitatively see whether the pattern observed at the central plane (X-Y axis) was also consistent along the depth (Z-axis); the droplets were subsequently imaged at three equally spaced planes along the Z-axis. The plane closest to the central plane (plane 3 ) was chosen at a distance $r / 3$. The next two planes (plane 2 and plane 1) were taken at distances $2 r / 3$ and $r$, respectively where $r \approx$ radius of the droplet. This indicates that the plane 1 chosen was very close to the periphery of the droplet along Z-axis. However, for FTIRM studies, only the central plane could be analyzed (by visually adjusting the focal plane at the largest observable size) because of the instrumental limitations (no depth scanning could be performed).

\section{Fluorescence lifetime imaging (FLIM) of $\alpha$-Syn liquid droplets}

$200 \mu \mathrm{M}$ of fluorescein-5-maleimide labeled (10\% v/v labeled and 90\% v/v unlabeled) $74 \mathrm{C}-\alpha-\mathrm{Syn}$ was phase-separated in presence of 10\% w/v PEG-8000 (in $20 \mathrm{mM}$ sodium phosphate buffer, $\mathrm{pH}$ 7.4 with $0.05 \%$ sodium azide) at $37^{\circ} \mathrm{C}$. The LLPS solution $(10 \mu \mathrm{l})$ was drop-casted onto a clean glass slide and sandwiched with an $18 \mathrm{~mm}$ coverslip (Blue Star, India). The edge of the coverslip was sealed with commercially available nail-polish. These slides were used for subsequent FLIM imaging at different time-points. Furthermore, identical samples were also incubated in $2 \mathrm{ml}$ eppendorf tubes and subjected to FLIM imaging by drop-casting onto clean glass slides just before measurements (Supplementary Fig. 5). FLIM was chosen because the technique is able to detect 
differences in fluorescence lifetime during protein aggregation and can provide a microscopic image of the sample with a spectrum of color coded pixels ${ }^{7}$. The spectrum of the color coded pixels indicates mean lifetime $\left(\tau_{\mathrm{m}}\right)$ values (blue being the lowest $\tau_{\mathrm{m}}$ and red being the highest $\tau_{\mathrm{m}}$ ). Moreover, fluorescein was chosen for our experiment as it shows concentration dependent changes of the intensity ${ }^{8}$, which was expected to help delineating the differences in $\tau_{\mathrm{m}}$ across different regions of phase-separated $\alpha$-Syn droplets. FLIM images of droplets were obtained at regular time intervals (d2-d20) with a time-resolved microscopy setup (MT-200, PicoQuant; Germany). The phase-separated samples were excited with a $482 \mathrm{~nm}$ laser $(5-8 \mu \mathrm{W})$ pulsing at $40 \mathrm{MHz}$. From the FLIM images, the fluorescence intensity decay profiles at different regions (C, IC OC and P) of the droplets were recorded from individual pixels under "single-point" recording mode. The fluorescence intensity decay was detected with a dual-band dichroic 480/645 (Chroma) in epifluorescence mode. The droplets were imaged at various Z-planes (planes $1 \rightarrow 3$ ) to obtain information along the depth (Z-axis) as mentioned earlier. All the FLIM images and data acquisition was performed using the in-built SymPhoTime 64 software (PicoQuant, Germany).

\section{Analysis of mean fluorescence lifetime}

Fluorescence intensity decay curves were fit with sum of exponential decay equations ${ }^{7,9}$;

$$
I(t)=\sum_{i=1}^{n} \alpha_{i} e^{-\left(\frac{\mathrm{t}}{\tau_{i}}\right)}
$$

Where $I(t)=$ emission fluorescence intensity at magic angle $\left(54.7^{\circ}\right)$ at time $\mathrm{t}$, $\alpha_{i}=$ amplitude of the fluorescence lifetime given that there are $i$ number of lifetimes, $\tau_{i}=i^{\text {th }}$ fluorescence lifetime where, $\Sigma \alpha_{i}=1$.

The fluorescence intensity decay curves obtained from the droplets were satisfactorily fit with biexponential equation ${ }^{7,9}$ using SymPhoTime 64 software (PicoQuant, Germany). Therefore, for our experiment, equation 1 can be represented as;

$$
I(t)=\alpha_{1} e^{-\left(\frac{\mathrm{t}}{\tau_{1}}\right)}+\alpha_{2} e^{-\left(\frac{\mathrm{t}}{\tau_{2}}\right)}
$$

From equation 2 , the mean fluorescence lifetime $\left(\tau_{m}\right)$ was determined using the following equation $^{10}$

Where,

$$
\tau_{m}=\alpha_{1} \tau_{1}+\alpha_{2} \tau_{2}
$$

$$
\alpha_{1}+\alpha_{2}=1
$$

For each sample, the mean long $\left(\Lambda_{\mathrm{m}}\right)$ fluorescence lifetime and mean short $\left(\lambda_{\mathrm{m}}\right)$ fluorescence lifetime were computed. Since we did not know the origin of the long and short lifetimes, the overall mean fluorescence lifetime $\left(\tau_{\mathrm{m}}\right)$ was calculated from each of the data-points on the droplets as a relative measure of our observations. Each region was denoted with different color codes ( $\mathbf{\square})$ with respect to its $\tau_{\mathrm{m}}$. The color codes were then assigned to the respective data-points to generate a spatially resolved map (SRM) of the liquid droplets.

For two fusing droplets at d5, multiple data-points were chosen on the FLIM image across the longitudinal fusion axis $(\mathrm{p} 1 \rightarrow \mathrm{p} 6)$. Although fusion is a dynamic process, the timescale of fusion 
(minutes) was much larger than the timescale of FLIM image acquisition (seconds) — enabling it to be experimentally feasible. From each of these data-points, the respective fluorescence intensity decay was obtained. The decays were subsequently fit to bi-exponential function to compute the $\tau_{\mathrm{m}}$ values. All the experimental and instrument parameters were identical and the data analyses were performed as described in the previous section.

\section{FTIRM imaging of $\alpha$-Syn liquid droplets}

Fourier transform infra-red microscopy (FTIRM) was performed with unlabeled WT $\alpha$-Syn protein. $200 \mu \mathrm{M}$ of unlabeled $\alpha$-Syn was mixed with $10 \%$ (w/v) PEG-8000 (in $20 \mathrm{mM}$ sodium phosphate buffer, $\mathrm{pH} 7.4$, with $0.05 \%$ sodium azide) and incubated at $37{ }^{\circ} \mathrm{C}$ to initiate LLPS. The LLPS solution was drop-casted onto a clean glass slide (Blue Star, India) and the formation of liquid droplets was confirmed by visualizing the LLPS solution under a DMi8 microscope (Leica Microsystems, Germany) in DIC mode. At different time intervals (d2-d20) during liquid-to-solid transition, the solution was spotted on $\mathrm{BaF}_{2}$ coated glass slide $(38 \times 19 \times 4 \mathrm{~mm})$ (Technosearch Instruments, India). $\mathrm{BaF}_{2}$ coated slide was used since it shows minimal absorption property in the entire UV-IR spectrum (200 $\mathrm{nm}$ to $12 \mu \mathrm{m}$ ) and is resistant towards high energy radiation. The experiment was performed in the presence of a Vertex-80v vacuum optics bench (Bruker, Germany), to minimize the presence of atmospheric water during FTIR spectrum acquisition. The liquid droplets were visualized and imaged at their central plane by manually focusing the droplet image until the largest diameter (size) is achieved by a 3000 Hyperion microscope attached with Vertex-80 FTIR machine (Bruker, Germany) at 20X magnification (ATR objective lens - $0.5 \mu \mathrm{m}$ resolution). Individual data-points were assigned on the FTIRM image of the droplets at different locations (C-Center, IC-Inner Circle, OC-Outer Circle and P-Periphery). However, due to the instrumental limitations, images along the Z-plane could not be obtained for FTIRM technique. Due to the smaller size of the liquid droplet $(2-3 \mu \mathrm{m})$ at $\mathrm{d} 2$, the data-points were obtained from all the pixels representing a single liquid droplet. FTIR spectra were recorded in "single-point mapping" mode 11,12 in the wavenumber range of $1500-1800 \mathrm{~cm}^{-1}$. This corresponds to the amideI ( $\mathrm{C}=\mathrm{O}$ stretching) and amide-II ( $\mathrm{C}-\mathrm{N}$ stretching \& $\mathrm{N}-\mathrm{H}$ bending) regions of a peptide bond showing predominant absorption intensity in that wavenumber range. Notably, the widely used "focal plane array" (FPA) mode ${ }^{11-13}$ could not be used for our experiments because the droplet size was very close to the resolution limit of the FTIRM $(0.5 \mu \mathrm{m})$ at earlier time-points. Therefore, to maximize the signal to noise ratio, single-point mapping mode was chosen for our study.

\section{FTIR spectra analysis}

FTIR spectra analysis was done with OPUS-65 v6.5 software. For each data point, background correction was implemented to eliminate the contributions from the solvent (and water) near 1650 $\mathrm{cm}^{-1}$. Subsequently, the background corrected spectra were subjected to baseline correction and "Fourier Self Deconvolution" (FSD) in the wavenumber range of 1600-1700 $\mathrm{cm}^{-1}$ corresponding to the amide-I $(\mathrm{C}=\mathrm{O}$ stretching) frequency. This was done because all of the protein secondary structures correspond to amide-I stretching in FTIR ${ }^{14}$. The deconvolution technique was performed 
to obtain single sharp peaks for each secondary structure which were masked by the convoluted (broadened) spectra. The FSD was performed using the Fourier transformed Lorentzian deconvolution function ${ }^{15}$. Furthermore, the deconvolution was performed by feeding band and noise deconvolution factors ${ }^{15}$ to the OPUS-65 v6.5 software. These factors were optimized to obtain maximum sharpness and minimum noise. After that, every peak maxima corresponding to different secondary structures were assigned on the deconvoluted spectra. The spectra were best fitted by auto-fitting method with minimum residual root mean square (RMS) error. The integrated area under each individual spectrum represented the fractional contribution from corresponding secondary structure. The integration limit was taken from $1600 \mathrm{~cm}^{-1}$ to $1700 \mathrm{~cm}^{-1}$, since all of the secondary structures appeared within the amide-I stretching frequency ${ }^{14,15}$. Each of the integration values was divided from the sum of all the integration values and multiplied with 100 to obtain the $\%$ abundance of each secondary structure. To schematically represent our findings, we assigned various color codes (represented with $\mathbf{m}$; one for each data-point) to denote the prevalent secondary structural combinations at different regions of the droplet with time. To conclusively state our observations, we took a fairly large sample-size ( $n=15$ droplets) for our FTIRM experiment.

To understand the secondary structure of $\alpha$-Syn at different locations of two fusing droplets (d5), the fusion event was imaged and three data-points (p1, p2 and p3) were assigned along the longitudinal fusion axis. The FTIR spectra were obtained from these data-points and subsequently analyzed as mentioned earlier. This could be possible because the time of acquisition of FTIRM spectra from a fusing droplet was in the time-range of seconds; which was much less than the timescale of fusion event (in minutes).

\section{In vitro fluorescence recovery after photobleaching (FRAP) of $\alpha$-Syn liquid droplets}

$200 \mu \mathrm{M}$ NHS-rhodamine labeled $(10 \% \mathrm{v} / \mathrm{v}$ labeled $+90 \% \mathrm{v} / \mathrm{v}$ unlabeled $) \alpha$-Syn was phaseseparated in the presence $10 \%$ PEG-8000 (in 20 mM sodium phosphate buffer, pH 7.4, 0.05\% sodium azide). The LLPS solution was drop-casted onto clean glass slides (Blue Star, India) and sandwiched with $18 \mathrm{~mm}$ coverslip (Blue Star, India). At two different time-points (d5 and d20) during LLPS, photo-bleaching of the phase separated $\alpha$-Syn droplets undergoing fusion event was performed using a standard protocol ${ }^{3}$ with a confocal microscope with an in-built FRAP setup (Zeiss Axio-Observer Z1 microscope). Briefly, a 561 nm DPSS 561-10 laser (at 100\% laser power) was used to bleach the larger droplet between the two droplets undergoing fusion. The bleaching region (ROI-1, termed as R1) was chosen on the larger droplet. Another ROI (termed as R2) of the same radius $(\omega)$ was chosen on the smaller droplet. The post-bleach fluorescence intensity was recorded from both R1 and R2, simultaneously. The passive bleaching by the monitoring laser was recorded from a different droplet (not undergoing fusion). The background correction was performed from the droplet free region. All the experimental parameters were identical and data analyses were performed as described in the following section. All of the microscopic snapshots were obtained with a 1024 x 1024 frame size with 8 bit-depth. 
For spatially resolved FRAP experiments at different location in the same droplet; d5, d7 and d15 LLPS samples (incubated in $2 \mathrm{ml}$ eppendorf tubes) were spotted onto clean glass slides (Blue Star, India) and immediately imaged under a confocal microscope with 63x oil immersion objective (Zeiss Axio-Observer Z1 microscope). The bleaching regions R1, R2 and R3 were chosen at the $\mathrm{C}, \mathrm{OC}$ and $\mathrm{P}$ regions, respectively and the fluorescence recovery was recorded for a span of $200 \mathrm{~s}$. The laser intensity and relevant instrumental parameters were kept identical for all time-points and for all bleaching regions. The microscopic snapshots were obtained with a 1024 x 1024 frame size with 8 bit-depth.

\section{FRAP data analysis}

Recovery of fluorescence obtained from different ROIs (R1, R2, and R3) were corrected at each time-frame for background and passive bleaching. This was done by computing the normalized fluorescence intensity, $I(n)$ using the following equation ${ }^{3}$;

$$
I(n)=\frac{[I(t)-I(b)]}{r}
$$

Where, $I(t)=$ fluorescence intensity at time $\mathrm{t}$,

$I(b)=$ background fluorescence intensity,

$r=I_{c} / I_{c_{0}}$ which is the rate of photo bleaching,

Where, $I_{c_{0}}=$ fluorescence intensity of the given ROI before photo-bleaching,

$I_{C}=$ fluorescence intensity of the given ROI after photo-bleaching.

From equation 9, the mobile fraction (M.F or $A$ ) of the protein molecule in the given ROI can be calculated using the following equation ${ }^{3}$;

$$
A=\frac{I_{\infty}-I_{C}}{I_{c_{0}}-I_{C}}
$$

Where, $I_{\infty}=$ fluorescence intensity at the end of recovery time-period

The normalized and background corrected fluorescence recovery was fitted using a single exponential equation with the help of OriginPro 9.0 (OriginLab, USA) software. The equation is as follows ${ }^{3,16-20}$;

$$
I(t)=A\left(1-\exp \left(\frac{-\mathrm{t}}{\tau}\right)\right)+C
$$

Where, $\tau=$ fluorescence recovery time constant,

$A=$ mobile fraction of the fluorescent probe,

$C=\mathrm{y}$-intercept of the recovery curve (if any).

\section{Spatially (and spectrally) resolved FRET microscopy}

Spectrally resolved FRET microscopy was performed by following a previously established protocol $^{3,21}$. Briefly, LLPS solution $(200 \mu \mathrm{M}$ protein $+10 \%$ PEG-8000) of fluorescein-5maleimide (Donor, $D$ ) labeled (10\% v/v labeled) 74C- $\alpha$-Syn was mixed equal volume of LLPS solution of rhodamine-C2-maleimide (Acceptor, $A$ ) labeled (10\% v/v labeled) $74 \mathrm{C}$ - $\alpha$-Syn. The solution was drop-casted on a clean glass-slide and sandwiched with an $18 \mathrm{~mm}$ coverslip and sealed for phase separation. Only $D$-labeled 74C- $\alpha$-Syn and only $A$-labeled $74 C-\alpha$-Syn was also phase separated simultaneously, which served as $D$ only and $A$ only controls, respectively. 
Spectrally resolved images of the droplets were obtained from both FRET and control samples at d2 (immediately after formation of liquid droplets), d5 (initial stage during liquid-to-solid transition) and d20 (final stage during liquid-to-solid transition). The images were acquired manually at the central plane using an epifluorescence total internal reflection fluorescence microscope (Nikon Eclipse TE2000-U). $D$ and $A$ were excited with $488 \mathrm{~nm}$ and $532 \mathrm{~nm}$ DPSS lasers (OXXIUS, model: ACX-CTRB and LASERGLOW, model: LRS-0532-PFM-00200-03), respectively. Single droplets were visualized with a 60X TIRF objective lens (1.49 NA) (Nikon, Japan). The co-localization of $D$ and $A$ labeled droplet was obtained by emissions at two energetically separated detection channels $(515-565 \mathrm{~nm}$ and $590-700 \mathrm{~nm}$ band-pass emission channels). The droplets were imaged at $50 \mathrm{~ms}$ exposure. A background flattening of each image was performed using ImageJ (Fiji, NIH). Spatially (as well as spectrally) resolved image of the droplets were acquired using a slit and a transmission grating $(70 \mathrm{~g} / \mathrm{mm})$ using a sCMOS camera (Hamamatsu ORCA-Flash 4.0 V3). The dispersed emission spectra (in the range 500-700 nm) from individual droplets were recorded in this manner. All the images were collected at identical excitation power $\left(5 \mathrm{~W} / \mathrm{cm}^{2}\right)$ of $488 \mathrm{~nm}$ and for same emission (at $532 \mathrm{~nm}$ ) exposure time $(0.3 \mathrm{~s})$. All measurements were performed at $25^{\circ} \mathrm{C}$ and the data were analyzed using ImageJ (Fiji, NIH) and OriginPro 9.0 (OriginLab, USA).

\section{FRET microscopy data analyses}

The data analysis of single droplet FRET measurement was performed according our previously established protocol ${ }^{3}$. Briefly, the $D$ only emission spectra were obtained by exciting the sample at $488 \mathrm{~nm}$ (donor $(D)$ excitation wavelength). The $A$ only droplets showed $\sim 5.4$ times greater fluorescence signal when excited at $532 \mathrm{~nm}$ (acceptor $(A)$ excitation wavelength) compared to 488 $\mathrm{nm}$ excitation. This was due to the difference of the absorption coefficients of $A$ at 488 and 532 $\mathrm{nm}$.

$$
0.185 \times \epsilon_{532 \mathrm{~nm}}^{\text {rhodamine }} \approx \epsilon_{488 \mathrm{~nm}}^{\text {rhodamine }}
$$

We also noticed a long tail in the emission spectrum of $D$, which was extended beyond the emission maxima of $A$ (at $595 \mathrm{~nm}$ ). Therefore, corrections for the emission tail and the difference in $\epsilon$ were performed according to our previously established protocol ${ }^{3}$. Subsequently, to calculate the apparent FRET efficiency, we defined a semi-quantitative term called the intensity enhancement factor due to the energy transfer $\left(I E F_{E T}\right)$, which was directly proportional to the actual FRET efficiency. The $I E F_{E T}$ accounted for both the corrections mentioned above.

Where,

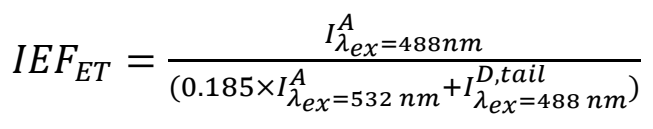

$I_{\lambda_{e x}=488 \mathrm{~nm}}^{A}=$ fluorescence intensity of $A$ when excited at $488 \mathrm{~nm}$,

$I_{\lambda_{e x} 532 \mathrm{~nm}}^{A}=$ fluorescence intensity of $A$ when excited at $532 \mathrm{~nm}$, $I_{\lambda_{e x}=488 \mathrm{~nm}}^{D, \text { tail }}=$ fluorescence intensity of the $D$ emission tail when excited at $488 \mathrm{~nm}$ for the FRET samples. 
In an actual FRET scenario, we can expect a positive enhancement of $I E F_{E T}$ (i.e. $I E F_{E T}>1$ ). The instrumental parameters were identical for the FRET samples as well as for $D$ only and $A$ only controls. The concentrations of the fluorescent dyes were also equal in both controls and FRET samples.

\section{Electron Microscopy (EM) of $\alpha$-Syn liquid droplets}

TEM imaging was done following a previously established protocol ${ }^{3}$. Briefly, $200 \mu \mathrm{M}$ of unlabeled $\alpha$-Syn was mixed with 10\% (w/v) PEG-8000 (in $20 \mathrm{mM}$ sodium phosphate buffer, $\mathrm{pH} 7.4,0.05 \%$ sodium azide) and incubated at $37{ }^{\circ} \mathrm{C}$ to initiate LLPS. The LLPS solution was drop-casted onto a clean glass slide (Blue Star, India) and sandwiched with an $18 \mathrm{~mm}$ coverslip (Blue Star, India). The formation of liquid droplets was confirmed by visualizing the LLPS solution under a DMi8 microscope (Leica Microsystems, Germany) in DIC mode. At different time-points (d2-d15), the coverslips were removed from the respective slides and the samples were transferred directly on the EM grid (Electron Microscopy Sciences, USA). The grids were negatively stained with aqueous $1 \%(\mathrm{w} / \mathrm{v})$ uranyl formate solution and subsequently imaged with a transmission electron microscope (Phillips CM-200, Amsterdam, Netherlands). The imaging was performed at $200 \mathrm{KV}$ and 6600X magnification and collected by Keen View Soft imaging system (Olympus, Tokyo, Japan). For scanning electron microscopy (SEM) experiments, the LLPS sample (d5) was dropcasted onto a clean glass coverslip and immediately imaged in cryo-mode with the help of a JSM7600F SEM (JEOL, Japan).

\section{TEM image analysis of $\alpha$-Syn liquid droplets}

The obtained TEM images at different time-points were analyzed with the help of ImageJ software (Fiji, NIH). A reference line along the entire diameter of the droplet was drawn on the TEM image and the grayscale intensity along the line was computed. Important to note, the pixel intensity values were higher at brighter regions of the image and lower at darker regions. Subsequently, angular maps ${ }^{22}$ of the droplets at individual time-points were generated by straightening the droplet image from 0 to $180^{\circ}$ with the help of an in-built "straighten" module in ImageJ (Fiji, NIH). To conclusively state our observations, a large number of sample-size was chosen for our analyses $(n=20$ droplets). 


\section{Supplementary Figures:}

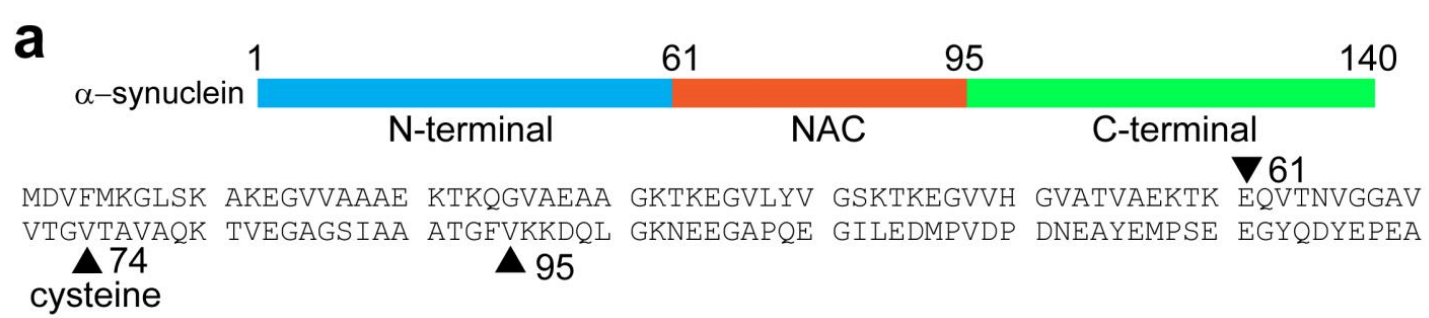

b
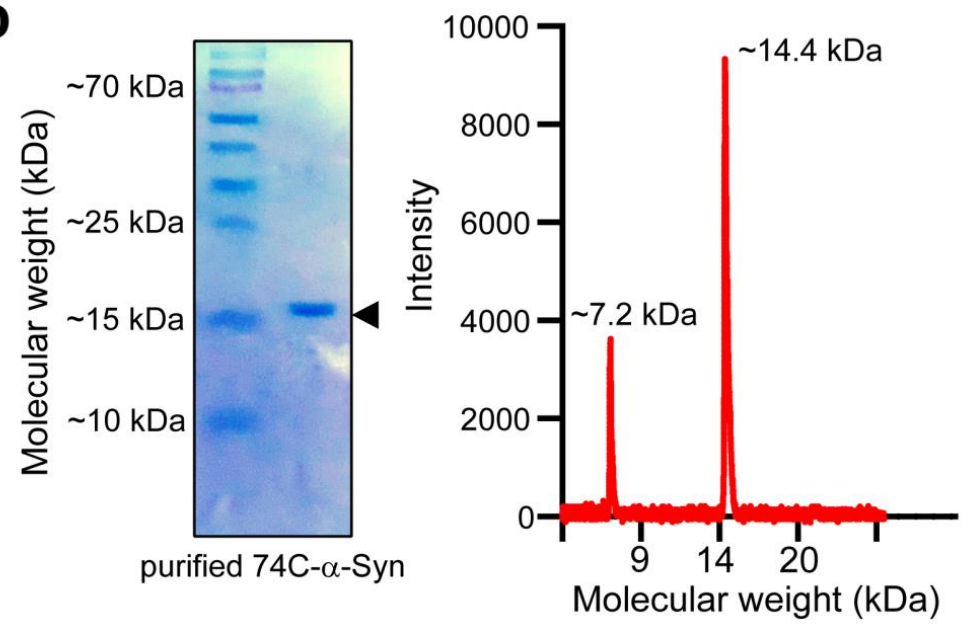

\section{Supplementary Fig. 1: Purification of 74C- $\alpha$-Syn for FLIM studies}

a. Upper panel: Schematic diagram showing the amphipathic N-terminal (1-60), hydrophobic NAC (61-95) and acidic C-terminal (96-140) domain of wild type (WT) human $\alpha$-Syn. Lower panel: The amino acid sequence of full length WT human $\alpha$-Syn is shown. The start and end of the hydrophobic NAC domain is denoted with black triangular pointers $(\boldsymbol{\Lambda})$ at residues 61-95, respectively. The $74^{\text {th }}$ valine residue $(\boldsymbol{\Delta})$ is mutated with cysteine to get the $74 \mathrm{C}-\alpha$-Syn mutant which has been used for FLIM and FRET studies. b. Left panel: SDS-PAGE of the purified 74C$\alpha$-Syn showing a single band at $\sim 15 \mathrm{kDa}$ representing the full length protein. Right panel: Mass spectrometry analysis also confirms that the purified $74 \mathrm{C}-\alpha-$ Syn is intact by showing two major peaks $(\mathrm{m} / \mathrm{z}-\sim 14.4 \mathrm{kDa}$ and $\mathrm{m} / 2 \mathrm{z} \sim 7.2 \mathrm{kDa})$. 
a

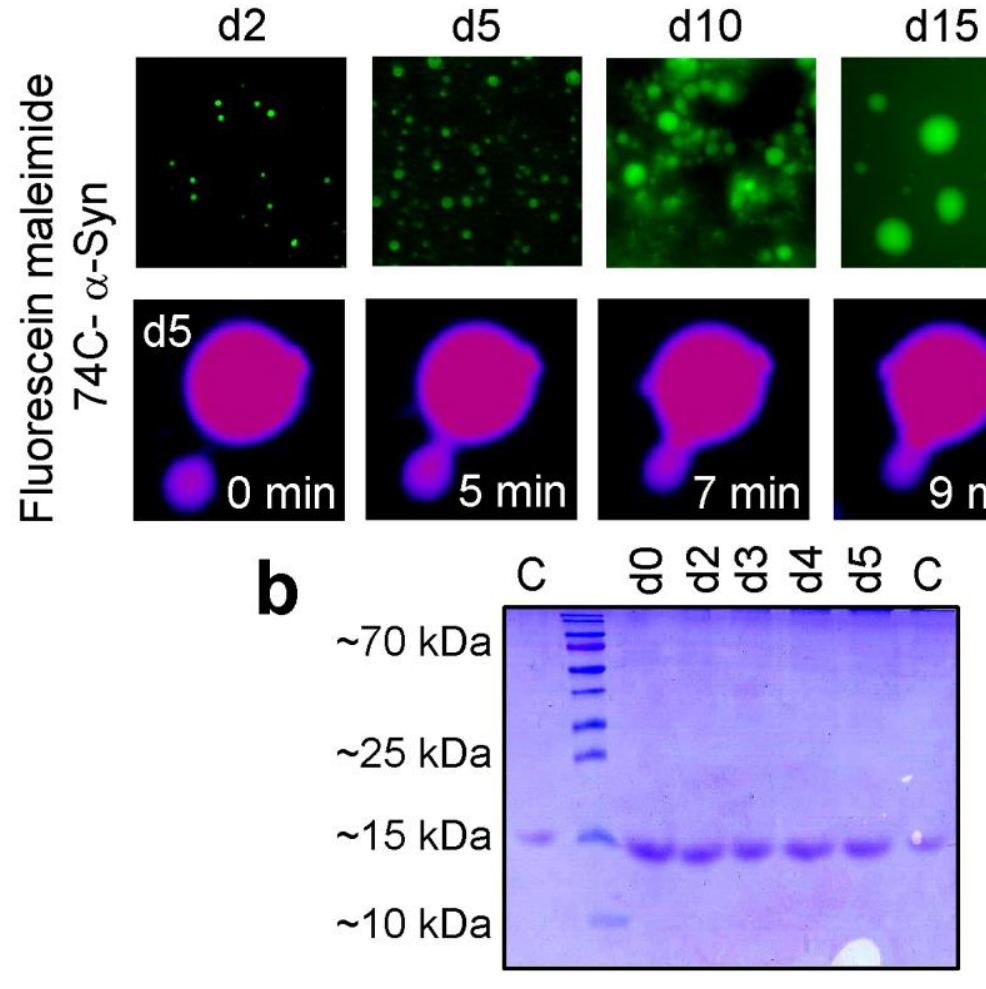

\section{Supplementary Fig. 2: LLPS behavior of labeled $\alpha$-Syn}

a. Upper panel: Fluorescein-5-maleimide labeled 74C- $\alpha$-Syn undergoes LLPS in presence of $10 \%$ PEG-8000. The droplets continuously grow both in size and number indicating the progression of LLPS. Representative images are shown. $n=3$ independent observations. Lower panel: Fusion event (at d5) between two fluorescein maleimide labeled $74 \mathrm{C}-\alpha$-Syn droplets is shown. Representative event is shown. The experiment is carried out two times with similar observations. The images are represented in thermal LUT for better visualization. b. SDS-PAGE of 74C- $\alpha-S y n$ at different time points $(\mathrm{d} 0-\mathrm{d} 5)$ showing that the protein remains intact during LLPS. " $\mathrm{C}$ " represents monomeric $74 \mathrm{C}-\alpha-\mathrm{Syn}$, which has been used as a control. 


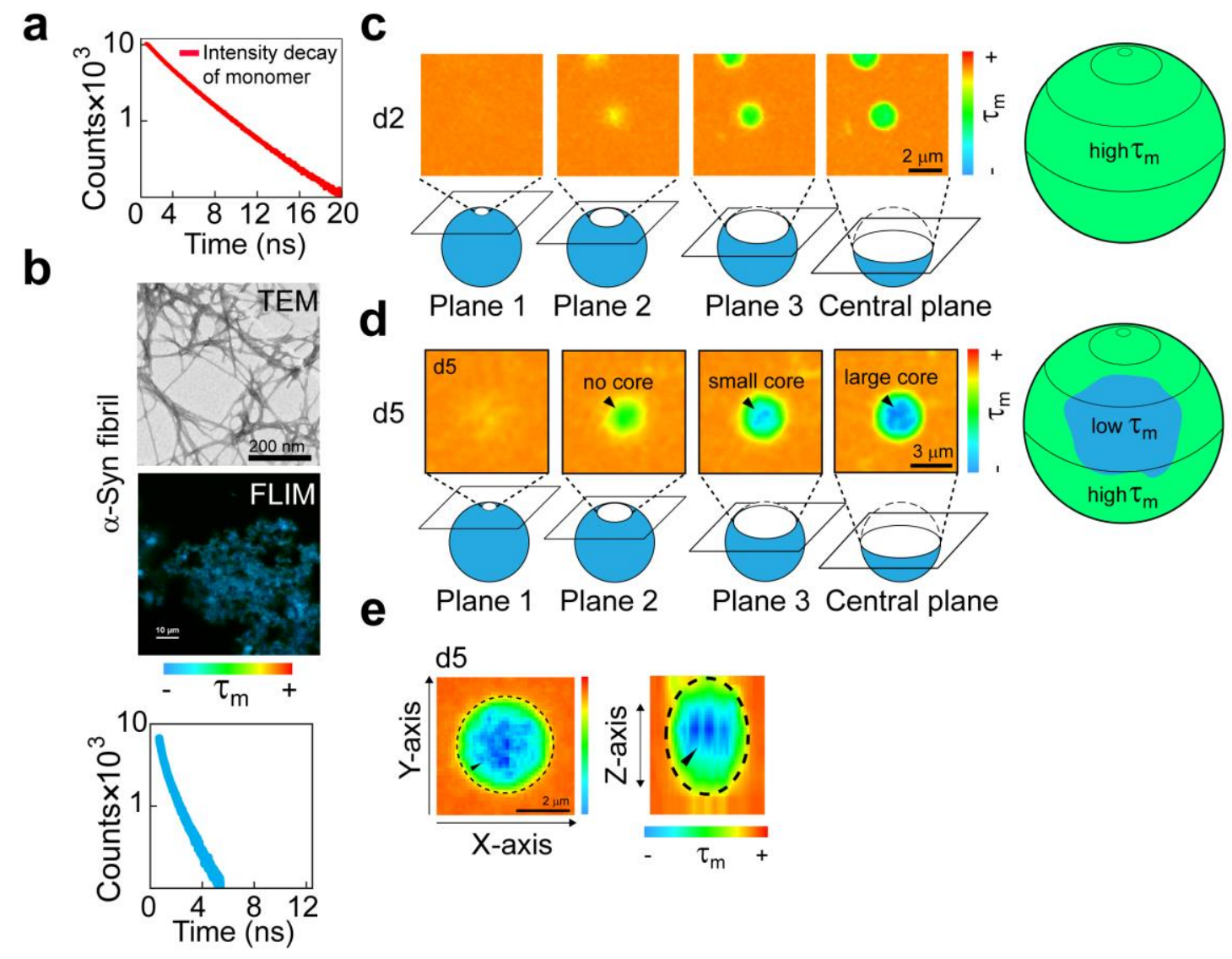

\section{Supplementary Fig. 3: Distribution of the mean fluorescence lifetime $\left(\tau_{\mathrm{m}}\right)$ of $\alpha$-Syn phase- separated droplets during liquid-to-solid transition}

a. Time-resolved fluorescence intensity decay of soluble, fluorescein-5-maleimide labeled 74C- $\alpha$ Syn monomer control. The overall mean lifetime $\left(\tau_{\mathrm{m}}\right)$ is calculated to be $\sim 4.1 \pm 1 \mathrm{~ns}$. Representative intensity decay profile is shown. The experiment is performed three times with similar results. $\mathbf{b}$. Upper panel: Transmission electron microscopy (TEM) imaging of independently prepared fluorescein-5-maleimide labeled 74C- $\alpha$-Syn amyloid fibrils. Representative images are shown. The experiment is performed three times with similar observations. Middle panel: Corresponding fluorescence lifetime images (FLIM) of the fibrils from which the time-resolved intensity decay is obtained. The color spectrum represents the range of mean lifetime $\left(\tau_{\mathrm{m}}\right)$ (blue being the lowest and red being the highest). Lower panel: Time-resolved fluorescence intensity decay of fluorescein5-maleimide labeled $74 \mathrm{C}-\alpha$-Syn fibril control. Representative decay profile is shown. The experiment is performed three times with similar results. c. Left panel: FLIM images of the droplets obtained at different Z-planes from the periphery to the center (plane $1 \rightarrow 3$, central plane) showing similar color image ( $\operatorname{similar} \tau_{\mathrm{m}}$ ) of $\alpha$-Syn at each plane in the $\mathrm{d} 2$ droplet. The FLIM image (top) and corresponding plane (bottom, schematically drawn) are shown. The FLIM images were manually brightened to highlight the differences of mean lifetimes across different regions. Right panel: Schematic diagram of a spherical d2 droplet showing similar life time $\left(\tau_{\mathrm{m}}\right)$ inside the droplet 
at different planes. d. Left panel: Fluorescence lifetime (FLIM) image of d5 droplets at different Z-planes (plane 1, 2, 3 and central plane) shows reduced $\tau_{\mathrm{m}}$ (blue color, denoted with black triangular pointers) when the Z-plane is close to the central plane (plane 3). For planes 1 and 2, which are away from the central plane (i.e. away from the center of the droplet), $\tau_{\mathrm{m}}$ becomes higher (green color). Right panel: Schematic diagram of a spherical d5 droplet showing the formation of a core structure at the center of the droplet, which has a different local microenvironment (reduced $\tau_{\mathrm{m}}$ ). The images are manually brightened to highlight the differences of mean lifetimes across different regions. e. FLIM images of planes $1 \rightarrow 3$ (along with the central plane) are superimposed. Orthogonal $(\mathrm{Z})$ projection of the superimposed image is shown along the Z-axis to show the spherical nature of the droplet. This data also confirms that the reduced $\tau_{\mathrm{m}}$ is indeed at the center (indicated by black triangular pointer $(\boldsymbol{\Delta})$ ) of a spherical, d5 liquid droplet. The images are manually brightened to highlight the differences of mean lifetimes across different regions. The black dashed line represents the boundary of the droplet. The experiment is performed three times with similar observations. 


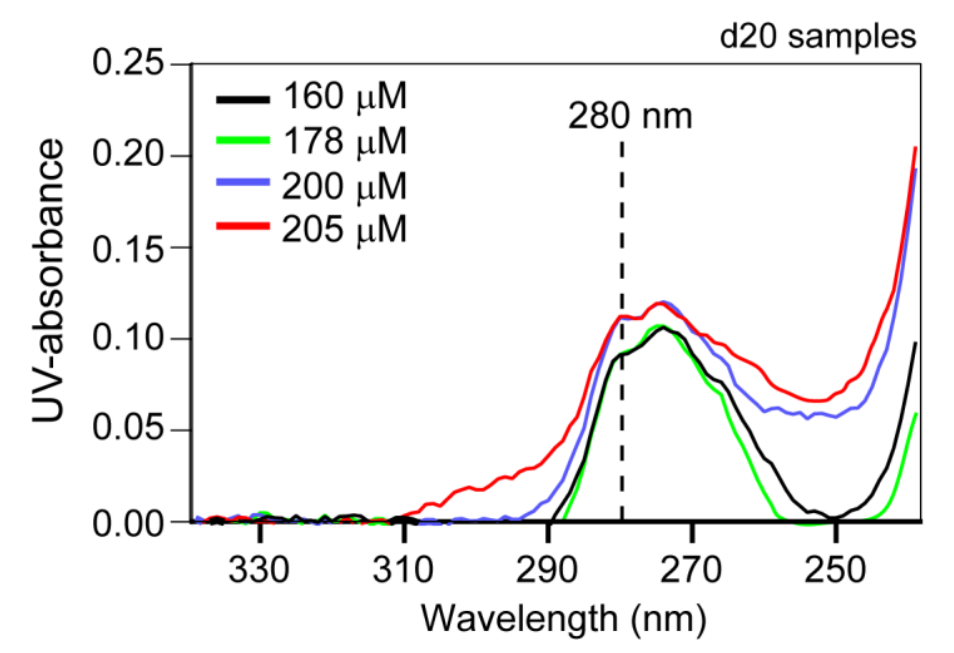

\section{Supplementary Figure 4: Incubation of LLPS sample on a slide does not lead to drying when kept in a moist chamber with frequent replenishment of moisture}

UV-Visible spectra of four different samples diluted from drop casted liquid droplet sample showing $>80 \%$ proteins are retained in the sample even after 20 days of incubation. This suggests that even after $\mathrm{d} 20$, the concentration of the LLPS samples do not change drastically from the original $200 \mu \mathrm{M}$ concentration. Even though the LLPS sample is heated at $90{ }^{\circ} \mathrm{C}$ for several minutes to denature any higher order aggregates, the hydrogel-like nature of the system after $\mathrm{d} 20$ may lead to inhomogeneous partitioning of the $\alpha$-Syn molecules while pipetting. Therefore, slight deviation (such as in the case of $160 \mu \mathrm{M}$ and $178 \mu \mathrm{M}$ ) from the original concentration may be observed. 

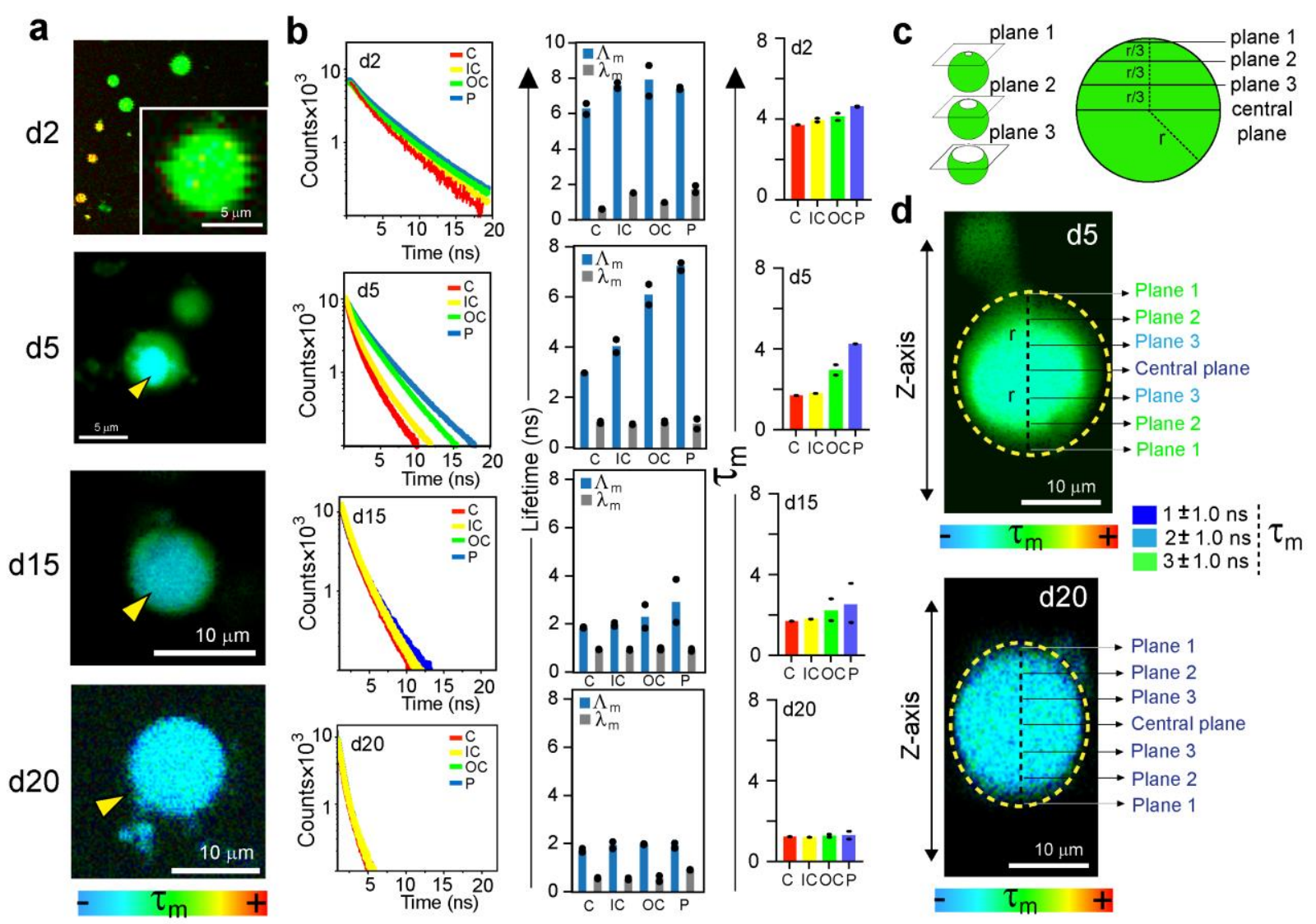

- Plane 1

$\rightarrow$ Plane 2

$\rightarrow$ Plane 3

Central plane

$\rightarrow$ Plane 3

$\rightarrow$ Plane 2

- Plane 1

Supplementary Figure 5: Changes in fluorescence lifetime initiates at the center and progresses towards the periphery of $\alpha$-Syn phase-separated droplets during liquid-to-solid transition when the LLPS samples are incubated in tubes

a. Representative color coded original FLIM images showing fluorescein-5-maleimide labeled $74 \mathrm{C}-\alpha-$ Syn droplets at various time points (d2-d20) during liquid-to-solid transition. The color spectrum represents the range of overall mean lifetime $\left(\tau_{\mathrm{m}}\right)$ (blue being the lowest and red being the highest). The images are acquired from the central plane. The yellow triangles mark the solidlike core at d5, d15 and d20. b. (Left panel) Time resolved fluorescence intensity decay obtained from different regions (C-Center, IC-Inner Circle, OC-Outer circle and P-Periphery) of the droplets at various time points $(\mathrm{d} 2-\mathrm{d} 20)$. Representative spectra are shown. The experiment is performed two times with similar observations. (Middle panel) The long $\left(\Delta_{\mathrm{m}}\right)$ and short $\left(\lambda_{\mathrm{m}}\right)$ lifetimes obtained from the intensity decay curved are plotted for C-Center, IC-Inner circle, OCOuter circle and P-Periphery at different time-points (d2, d5, d15 and d20). (Right panel) Corresponding mean lifetimes $\left(\tau_{\mathrm{m}}\right)$ obtained from intensity decay curve at different regions $(\mathrm{C}$ Center, IC-Inner circle, OC-Outer circle and P-Periphery) of the phase-separated droplets are plotted for different time-points (d2, d5, d15 and d20). c. Schematic showing analysis of droplets along the depth (Z-axis). Apart from the central plane, three other planes (plane 1, 2 and 3) along the Z-axis of the droplets are also imaged for FLIM to examine the distribution of lifetimes along 
the depth. The planes are equally spaced with a distance of $r / 3$ with radius of the droplet is $r$. d. (Upper panel) FLIM image of a d5 droplet along the Z-axis is shown. The mean lifetimes $\left(\tau_{\mathrm{m}}\right)$ calculated from different planes along the Z-axis confirms the presence of solid-like core at the center of the phase separated droplet. (Lower panel) FLIM image of a d20 droplet along the Z-axis is shown. The mean lifetimes $\left(\tau_{\mathrm{m}}\right)$ calculated from different planes along the Z-axis indicates the homogeneous nature of the droplet with a reduced $\tau_{\mathrm{m}}$ indicating the progression of solid-like core to the periphery of the droplet. The experiment is performed two independent times with similar observations. 
a

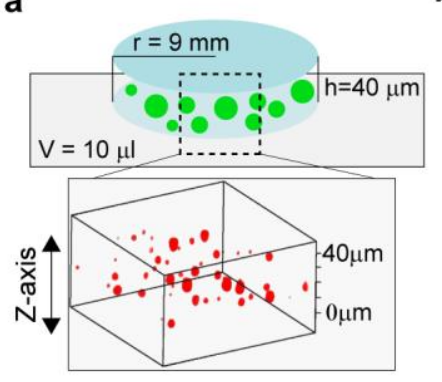

b

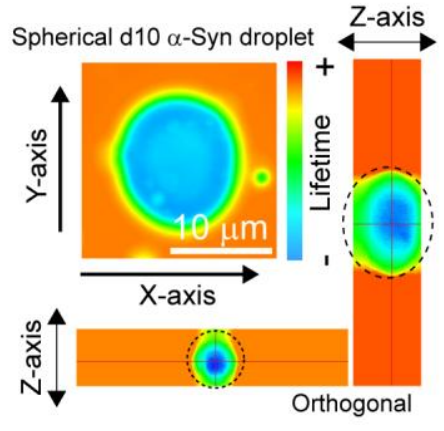

C Sedimented d $10 \alpha$-Syn droplet

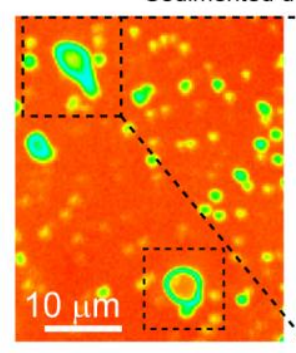

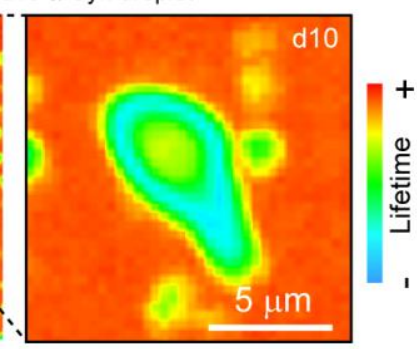

\section{Supplementary Figure 6: Analysis of $\alpha-S y n$ phase separated droplets that are geometrically spherical}

a. Schematic representation of the drop-casted phase separated sample, which creates a cylinder when sandwiched with a coverslip with a radius (r) of $9 \mathrm{~mm}$. The depth (h) of the cylinder is calculated to be $\sim 40 \mu \mathrm{m}$. Bottom panel represents a $3 \mathrm{~d}$-reconstitution of a real Z-scan data obtained from the microscope, which shows spherical d5 $\alpha$-Syn droplets in the solution. b. FLIM depth scanning (along Z-axis) of a d10 droplet containing a solid-like core (reduced lifetimeindicated with blue color) clearly shows the spherical nature of the droplet. c. At d10, some of the droplets sediment at the bottom of the glass slide and lose their spherical shape. Inset represents a d10 droplet showing a deformed lifetime distribution as well as geometry when it is in contact with the glass surface. Representative images are shown. 

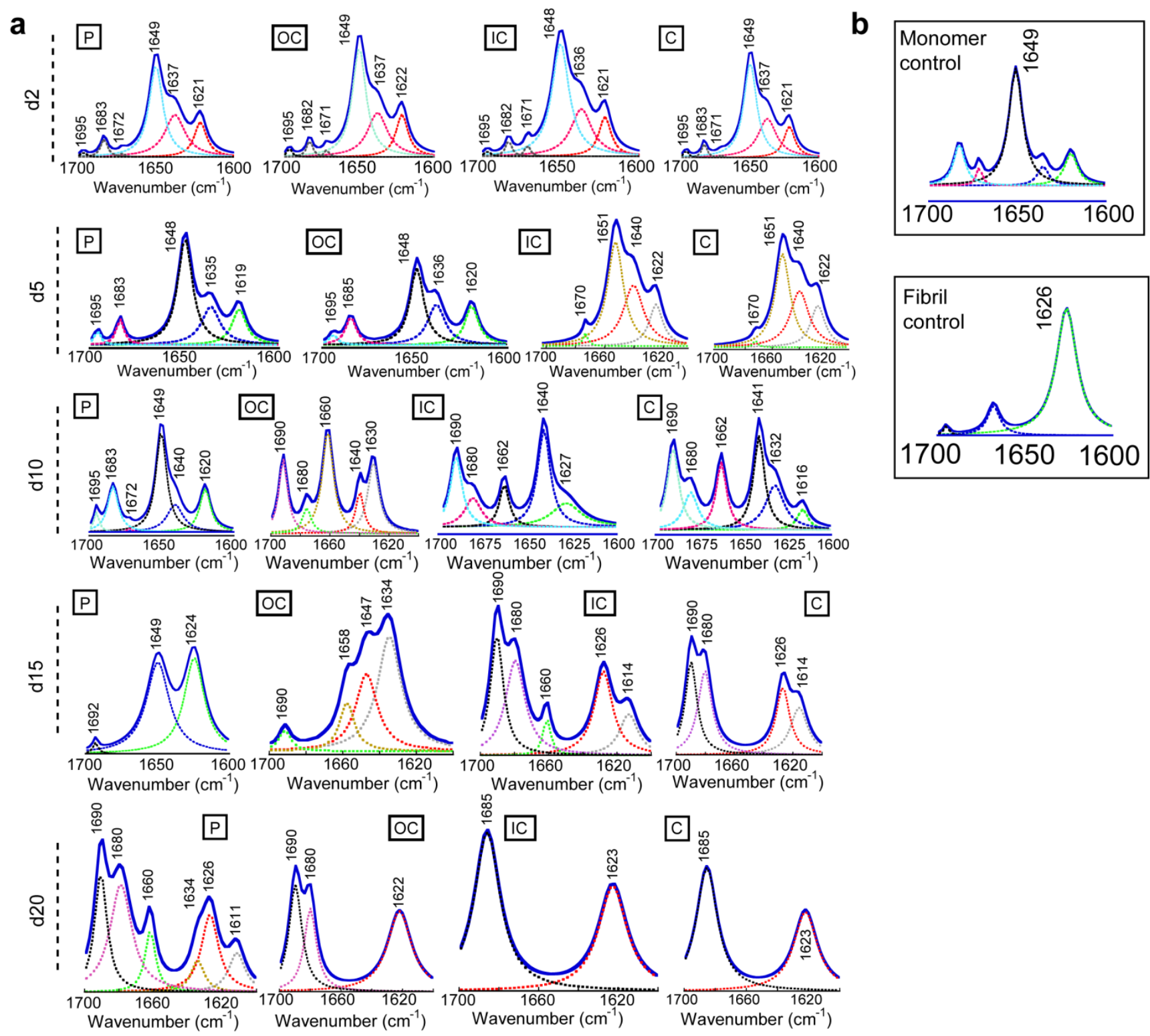

Supplementary Fig. 7: Fourier-transform infrared (FTIR) spectra of various regions of $\alpha$ Syn phase-separated droplet during liquid-to-solid transition

a. Representative FTIR spectra obtained from different regions (C-Center, IC-Inner Circle, OCOuter circle and P-Periphery) of the microscopic image of $\alpha$-Syn droplets at d2, d5, d10, d15 and d20 are shown. b. (Boxed): FTIR spectra of $\alpha$-Syn monomer (upper panel) and pre-formed $\alpha$-Syn fibril (lower panel) are shown as controls. The individual peaks corresponding to different secondary structures are assigned (dotted lines) on the deconvoluted spectra (solid blue line) (ab). The experiment is performed three times with similar results. 


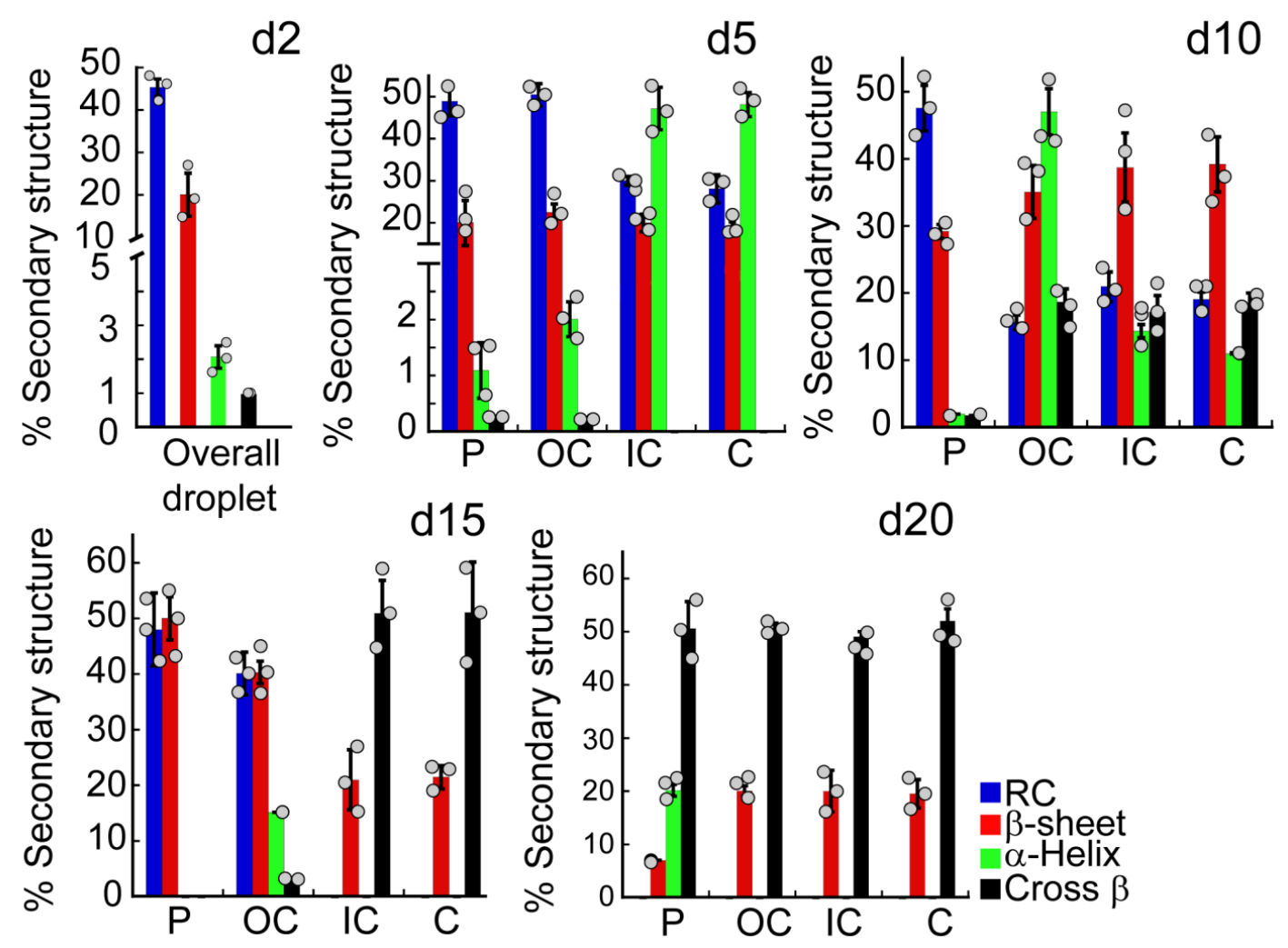

Supplementary Fig. 8: Conformational transition of $\alpha$-Syn inside the phase-separated droplets

FTIR spectra are deconvoluted and the \% of the secondary structure for different regions (CCenter, IC-Inner circle, OC-Outer circle and P-Periphery) on the droplets at various time-points $(\mathrm{d} 2, \mathrm{~d} 5, \mathrm{~d} 10, \mathrm{~d} 15$ and $\mathrm{d} 20)$ are determined. The $\%$ of random coil (RC), $\beta$-sheet, $\alpha$-helix and cross$\beta$ are plotted. Values represent mean \pm S.D for $\mathrm{n}=3$ independent experiments. 


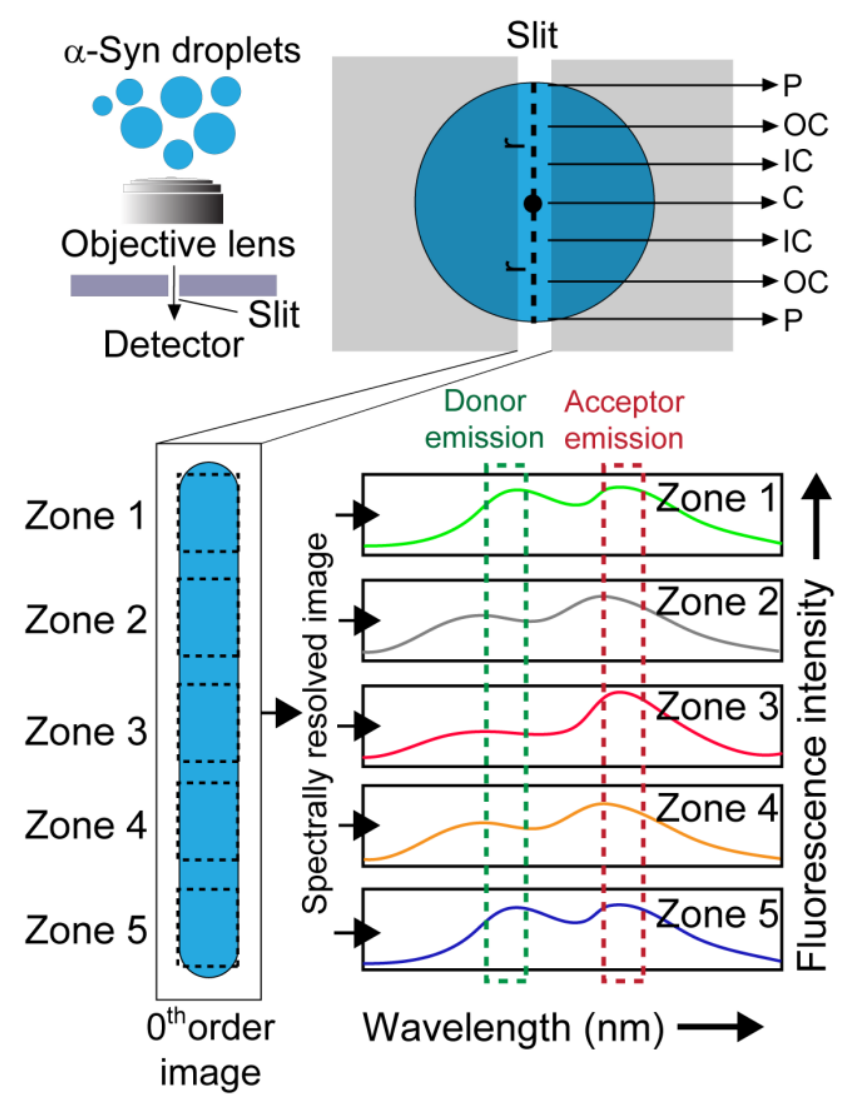

\section{Supplementary Fig. 9: Working principle behind spatially resolved FRET microscopy of $\alpha$ - Syn LLPS}

Schematic diagram of the working principle for FRET microscopy is shown. Top left: Phase separated $\alpha$-Syn droplets labeled with both fluorescein-5-maleimide (Donor, $D$ ) and rhodamineC2-maleimide (Acceptor, $A$ ) labeled 74C- $\alpha$-Syn, which is visualized under a coverslip through a narrow slit. Top right: The slit is positioned in such a way so that the droplet is visualized along its entire diameter (Radius $(\boldsymbol{r})+\boldsymbol{r}$ ). Different regions (C-Center, IC-Inner Circle, OC-Outer circle and P-Periphery) have been assigned along the diameter of the droplet. Bottom left: The microscopic image obtained by this alignment is called the $0^{\text {th }}$ order image ${ }^{3,21}$. The $0^{\text {th }}$ order image along the diameter can be imaged with the excitation wavelength of $D$ and emission wavelengths of both $D$ and $A$, in a location specific (Zones 1-5) manner. By doing this, we can generate a spatially as well as spectrally resolved image ${ }^{3}$ of the droplet. The fluorescence intensity profiles at different emission wavelengths for different locations (Zones 1-5) (Bottom right) enable us to calculate the intensity enhancement factor $\left(I E F_{E T}\right)$ which is a proportional measure of the FRET efficiency ${ }^{3}$. 


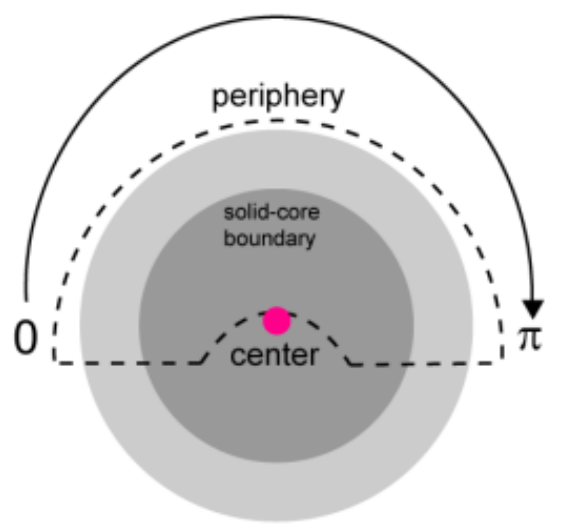

Straightened angular map (0 to $\pi$ )

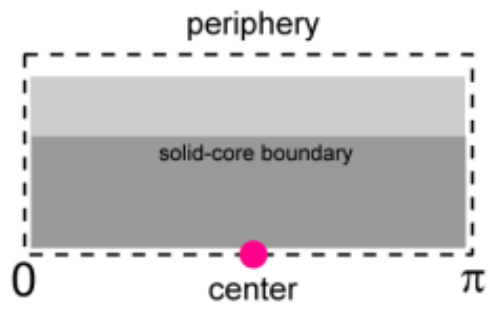

\section{Supplementary Fig. 10: Angular maps of TEM images of $\alpha$-Syn liquid droplets}

Left panel: Schematic representing a phase-separated $\alpha$-Syn droplet (light gray) containing a solidlike core (dark gray) observed under TEM. The black dashed boundary denotes the area (spanning $0 \rightarrow \pi$ or $0^{\circ} \rightarrow 180^{\circ}$ angle of the droplet) from which the angular map (right panel) has been generated $^{22}$. The center of the droplet is marked with a pink dot $(\bullet)$. Right panel: The straightened, angular map $\left(0 \rightarrow \pi\right.$ or $0^{\circ} \rightarrow 180^{\circ}$ angle $)$ of the droplet has been schematically represented. The center of the droplet is marked with a pink $\operatorname{dot}(\bullet)$. From here, the distance of the solid-like core boundary from the center is easily quantified with respect to the radius. 

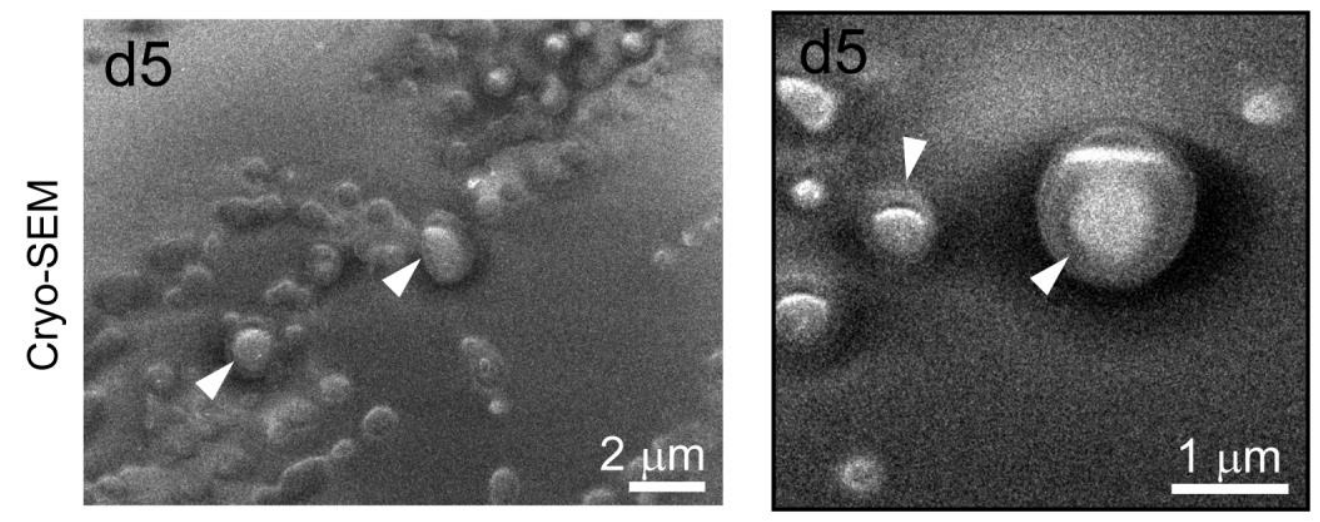

\section{Supplementary Figure 11: Cryo-SEM imaging of $\alpha$-Syn liquid droplets at d5}

Cryo-SEM imaging of d5 droplets showing the presence of a core structure (marked with white triangular pointers) supporting TEM observations of solid like dense structure at the center of liquid droplet. Representative images from two different experiments are shown. 
Supplementary Table 1: Deconvoluted amide-I stretching frequencies in the range of 1600-1700 $\mathrm{cm}^{-1}$ and their corresponding protein secondary structures ${ }^{14}$.

\begin{tabular}{|l|l|l|}
\hline Secondary Structure & Start wavenumber $\left(\mathrm{cm}^{-1}\right)$ & End wavenumber $\left(\mathrm{cm}^{-1}\right)$ \\
\hline Random coil $(\mathrm{RC})$ & $1640 \pm 2$ & $1648 \pm 2$ \\
\hline$\beta$-sheet & $1620 \pm 2$ & $1638 \pm 2$ \\
\hline$\alpha$-helix & $1650 \pm 2$ & $1660 \pm 2$ \\
\hline Cross- $\beta$ amyloid & $1690 \pm 2$ & $1695 \pm 2$ \\
\hline
\end{tabular}


Supplementary Table 2: The \% of the secondary structures of $\alpha$-Syn at different locations (CCenter, IC-Inner circle, OC-Outer circle and P-Periphery) of the phase-separated droplets during liquid-to-solid transition (d5-d20). The \% of turn and loop structures are not mentioned in the table.

\begin{tabular}{|l|l|l|l|l|l|}
\hline \multirow{4}{*}{ Time (days) } & $\begin{array}{l}\text { Secondary } \\
\text { structural } \\
\text { conformation }\end{array}$ & \multicolumn{4}{|l|}{$\begin{array}{l}\text { Percentage of different secondary structures at different } \\
\text { location inside the droplet }\end{array}$} \\
\cline { 2 - 6 } & P (\%) & OC (\%) & IC (\%) & C (\%) \\
\hline \multirow{5}{*}{ d5 } & RC & 48.9 & 50.4 & 30.0 & 28.0 \\
\cline { 2 - 6 } & $\beta$-sheet & 20.1 & 22.4 & 20.0 & 19.1 \\
\cline { 2 - 6 } & $\alpha$-helix & 1.1 & 2.0 & 47.2 & 48.1 \\
\cline { 2 - 6 } & Cross- $\beta$ & 0.3 & 0.2 & $\sim 0$ & $\sim 0$ \\
\hline \multirow{5}{*}{ d10 } & RC & 47.5 & 15.6 & 20.9 & 19.1 \\
\cline { 2 - 6 } & $\beta$-sheet & 29.2 & 35.1 & 38.7 & 39.2 \\
\cline { 2 - 6 } & $\alpha$-helix & 1.9 & 47.0 & 14.3 & 11.0 \\
\cline { 2 - 6 } & Cross- $\beta$ & 1.8 & 18.7 & 17.1 & 18.0 \\
\hline \multirow{5}{*}{ d15 } & RC & 48.0 & 40.1 & $\sim 0$ & $\sim 0$ \\
\cline { 2 - 6 } & $\beta$-sheet & 50.0 & 40.3 & 20.9 & 21.4 \\
\cline { 2 - 6 } & $\alpha$-helix & $\sim 0$ & 15.1 & $\sim 0$ & $\sim 0$ \\
\cline { 2 - 6 } & Cross- $\beta$ & $\sim 0$ & 3.1 & 50.9 & 51.0 \\
\hline \multirow{5}{*}{ d20 } & RC & $\sim 0$ & $\sim 0$ & $\sim 0$ & $\sim 0$ \\
\cline { 2 - 6 } & $\beta$-sheet & 7.0 & 20.0 & 20.0 & 19.5 \\
\cline { 2 - 6 } & $\alpha$-helix & 20.1 & $\sim 0$ & $\sim 0$ & $\sim 0$ \\
\cline { 2 - 6 } & Cross- $\beta$ & 50.5 & 50.5 & 49.0 & 52.0 \\
\hline
\end{tabular}


Supplementary Table 3: The $\%$ of the secondary structures of $\alpha$-Syn at different locations (p1contact region of fusion, p2-center of smaller droplet and p3-center of larger droplet) of the phaseseparated droplets undergoing fusion event at $\mathrm{d} 5$. The $\%$ of turn and loop structures are not mentioned in the table.

\begin{tabular}{|l|l|l|l|l|}
\hline \multirow{2}{*}{$\begin{array}{l}\text { Time } \\
\text { days })\end{array}$} & $\begin{array}{l}\text { Secondary } \\
\text { structural } \\
\text { conformation }\end{array}$ & $\begin{array}{l}\text { Percentage of different secondary structures at different location } \\
\text { inside the droplet undergoing fusion }\end{array}$ \\
\cline { 3 - 5 } & & $\mathrm{p} 1(\%)$ & $\mathrm{p} 2(\%)$ & $\mathrm{p} 3(\%)$ \\
\hline \multirow{3}{*}{ d5 } & RC & 50.1 & 49.2 & 28.2 \\
\cline { 2 - 5 } & $\beta$-sheet & 19.5 & 20.0 & 18.1 \\
\cline { 2 - 5 } & $\alpha$-helix & 0.9 & 1.6 & 50.8 \\
\cline { 2 - 5 } & Cross- $\beta$ & $\sim 0$ & $\sim 0$ & $\sim 0$ \\
\hline
\end{tabular}

\section{Supplementary video legends}

Supplementary video 1: Spatially resolved FRAP of d5 $\alpha$-Syn droplet confirm that the molecules at the center are less diffusive compared to the periphery due to the formation of a solid-like core. 


\section{References}

1 Volles, M. J. \& Lansbury, P. T., Jr. Relationships between the sequence of alphasynuclein and its membrane affinity, fibrillization propensity, and yeast toxicity. $J \mathrm{Mol}$ Biol 366, 1510-1522, doi:10.1016/j.jmb.2006.12.044 (2007).

2 Singh, P. K. et al. Curcumin modulates alpha-synuclein aggregation and toxicity. ACS Chem Neurosci 4, 393-407, doi:10.1021/cn3001203 (2013).

3 Ray, S. et al. $\alpha$-Synuclein aggregation nucleates through liquid-liquid phase separation. Nature Chemistry 12, 705-716, doi:10.1038/s41557-020-0465-9 (2020).

4 Winner, B. et al. In vivo demonstration that $\alpha$-synuclein oligomers are toxic. Proceedings of the National Academy of Sciences 108, 4194-4199, doi:10.1073/pnas.1100976108 (2011).

5 Ghosh, D. et al. Structure based aggregation studies reveal the presence of helix-rich intermediate during alpha-Synuclein aggregation. Sci Rep 5, 9228, doi:10.1038/srep09228 (2015).

6 Wilkins, M. R. et al. Protein identification and analysis tools in the ExPASy server. Methods Mol Biol 112, 531-552, doi:10.1385/1-59259-584-7:531 (1999).

$7 \quad$ Lakowicz, J. R. Principles of fluorescence spectroscopy. (2010).

8 Chen, R. F. \& Knutson, J. R. Mechanism of fluorescence concentration quenching of carboxyfluorescein in liposomes: Energy transfer to nonfluorescent dimers. Analytical Biochemistry 172, 61-77, doi:https://doi.org/10.1016/0003-2697(88)90412-5 (1988).

9 Sahay, S. et al. Familial Parkinson disease-associated mutations alter the site-specific microenvironment and dynamics of alpha-synuclein. J Biol Chem 290, 7804-7822, doi:10.1074/jbc.M114.598607 (2015).

10 Lakowicz, J. R. Principles of flourescence spectrocopy. 529-575 (2006).

11 Bhargava, R., Wall, B. G. \& Koenig, J. L. Comparison of the FT-IR Mapping and Imaging Techniques Applied to Polymeric Systems. Applied Spectroscopy 54, 470-479, doi:10.1366/0003702001949870 (2000).

12 Chan, K. L. A., Altharawi, A., Fale, P., Song, C. L. \& Kazarian, S. G. Transmission Fourier Transform Infrared Spectroscopic Imaging, Mapping, and Synchrotron Scanning Microscopy with Zinc Sulfide Hemispheres on Living Mammalian Cells at Sub-Cellular Resolution. 74, 544-552, doi:10.1177/0003702819898275 (2020).

13 Araki, K. et al. Synchrotron FTIR micro-spectroscopy for structural analysis of Lewy bodies in the brain of Parkinson's disease patients. Scientific Reports 5, 17625, doi:10.1038/srep17625 (2015).

14 Jackson, M. \& Mantsch, H. H. The use and misuse of FTIR spectroscopy in the determination of protein structure. Crit Rev Biochem Mol Biol 30, 95-120, doi:10.3109/10409239509085140 (1995).

15 Yang, H., Yang, S., Kong, J., Dong, A. \& Yu, S. Obtaining information about protein secondary structures in aqueous solution using Fourier transform IR spectroscopy. Nat Protoc 10, 382-396, doi:10.1038/nprot.2015.024 (2015).

16 Taylor, N. O., Wei, M. T., Stone, H. A. \& Brangwynne, C. P. Quantifying Dynamics in Phase-Separated Condensates Using Fluorescence Recovery after Photobleaching. Biophys J 117, 1285-1300, doi:10.1016/j.bpj.2019.08.030 (2019).

17 Patel, A. et al. A Liquid-to-Solid Phase Transition of the ALS Protein FUS Accelerated by Disease Mutation. Cell 162, 1066-1077, doi:10.1016/j.cell.2015.07.047 (2015). 
18 Elbaum-Garfinkle, S. et al. The disordered P granule protein LAF-1 drives phase separation into droplets with tunable viscosity and dynamics. Proc Natl Acad Sci U S A 112, 7189-7194, doi:10.1073/pnas.1504822112 (2015).

19 Banani, S. F. et al. Compositional Control of Phase-Separated Cellular Bodies. Cell 166, 651-663 (2016).

20 Aumiller, W. M., Jr., Pir Cakmak, F., Davis, B. W. \& Keating, C. D. RNA-Based Coacervates as a Model for Membraneless Organelles: Formation, Properties, and Interfacial Liposome Assembly. Langmuir 32, 10042-10053, doi:10.1021/acs.langmuir.6b02499 (2016).

21 Das, S., Sharma, D. K., Chakrabarty, S., Chowdhury, A. \& Sen Gupta, S. Bioactive Polymersomes Self-Assembled from Amphiphilic PPO-GlycoPolypeptides: Synthesis, Characterization, and Dual-Dye Encapsulation. Langmuir 31, 3402-3412, doi:10.1021/la503993e (2015).

22 Ianiro, A. et al. Liquid-liquid phase separation during amphiphilic self-assembly. Nature Chemistry 11, 320-328, doi:10.1038/s41557-019-0210-4 (2019). 
a

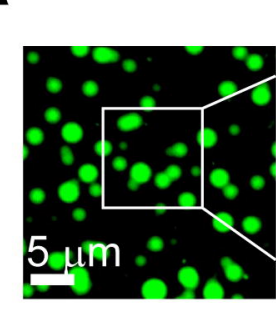

b
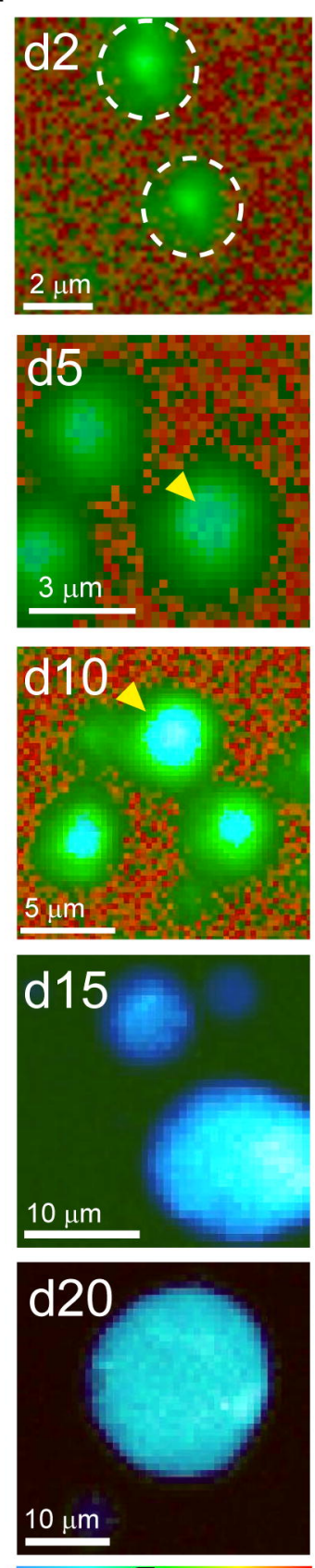

$\tau_{m}+$

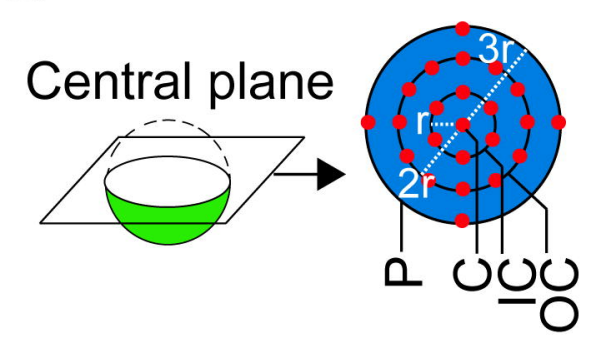

e

Fusion
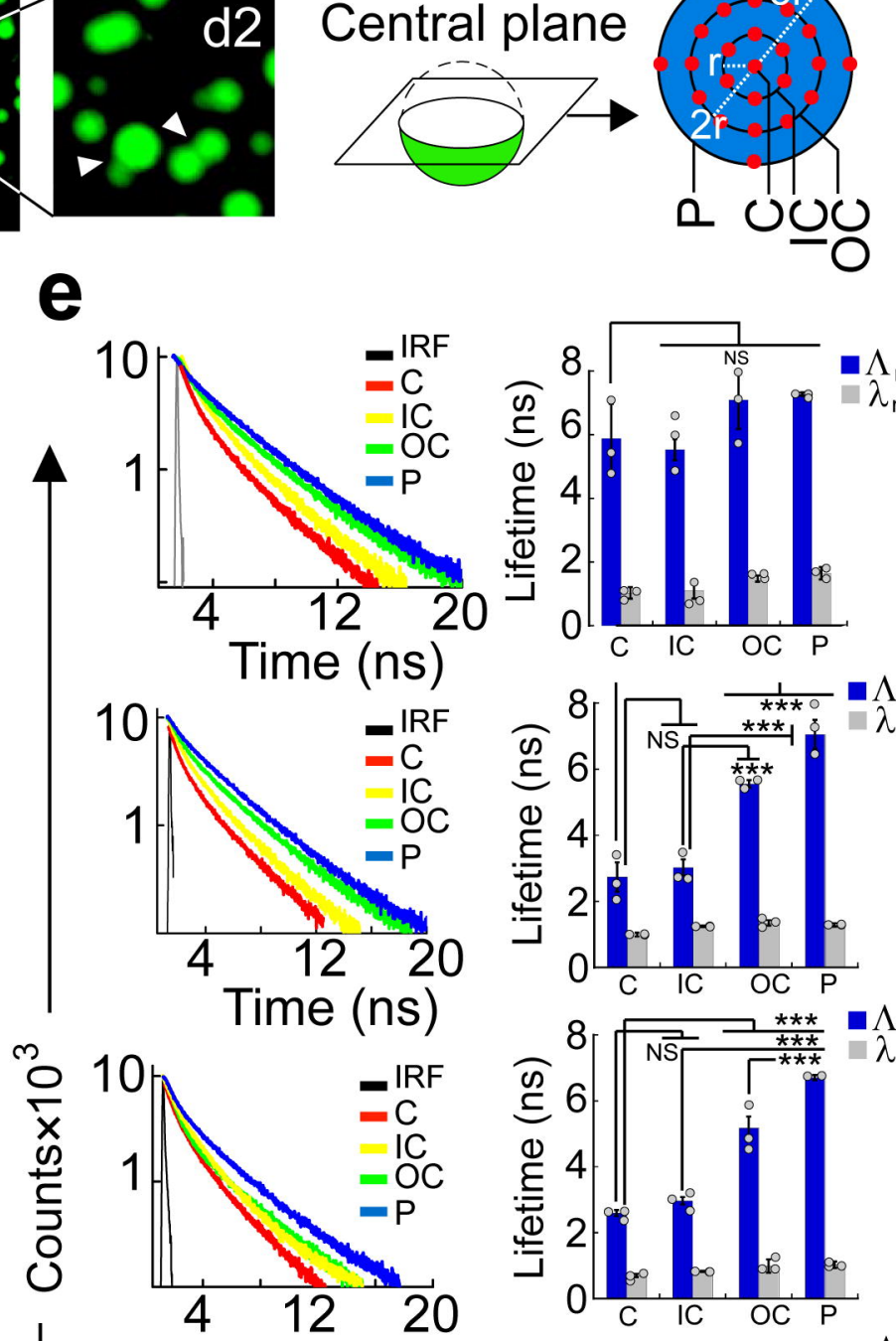

Time (ns)

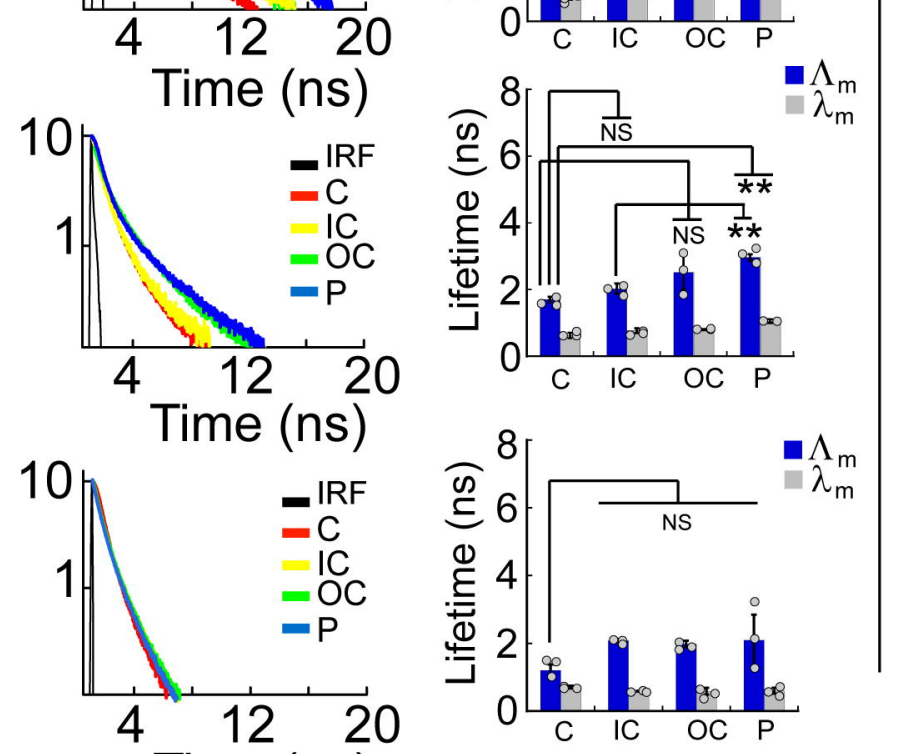
Time (ns)
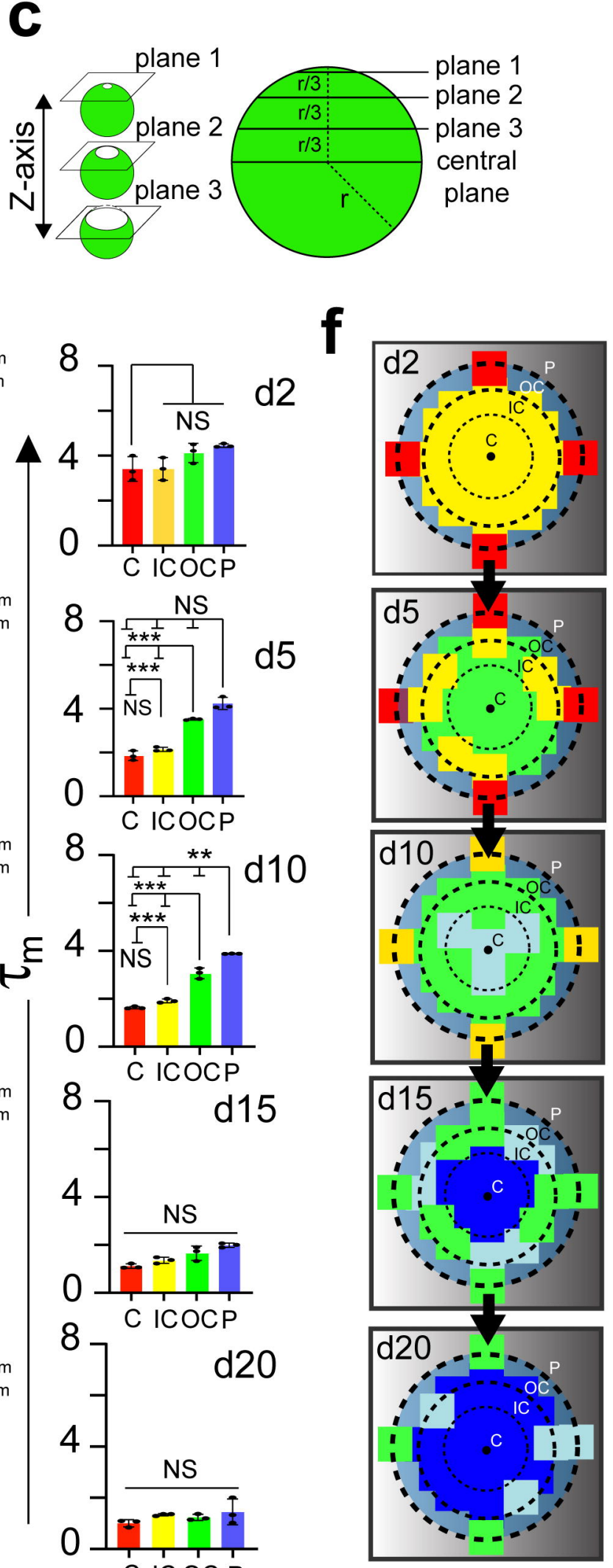

$1 \pm 0.2 \mathrm{~ns}$ $1 \pm 0.5 \mathrm{~ns}$

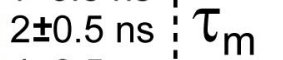
$4 \pm 0.5 \mathrm{~ns}$ $5 \pm 0.5$ ns 
$a$

d2
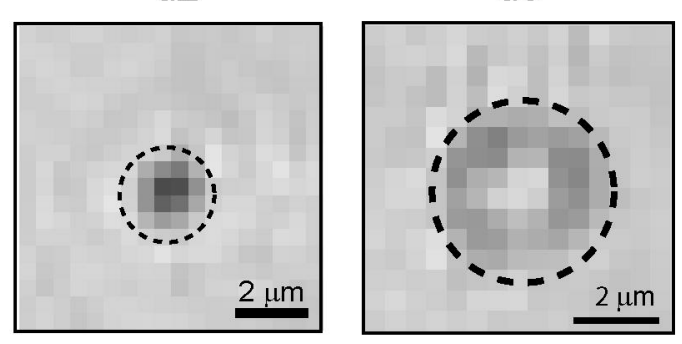

d10

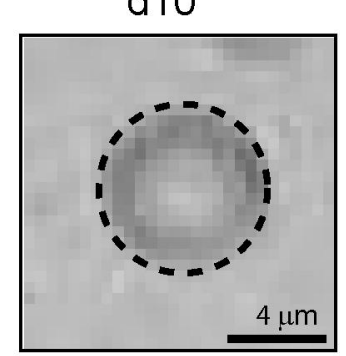

d15

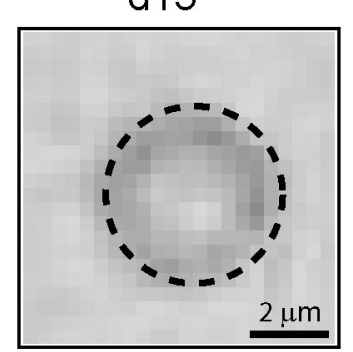

d20

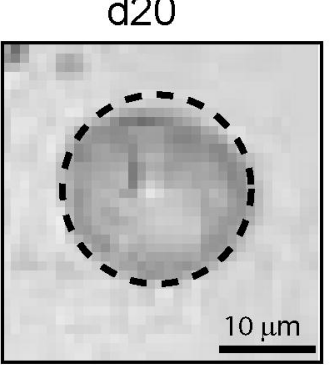

b

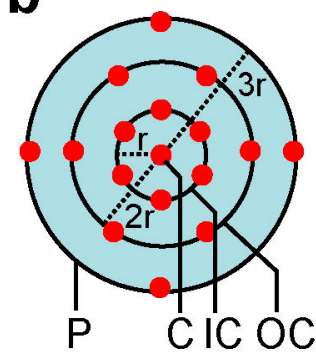

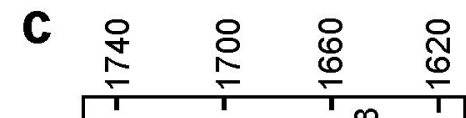

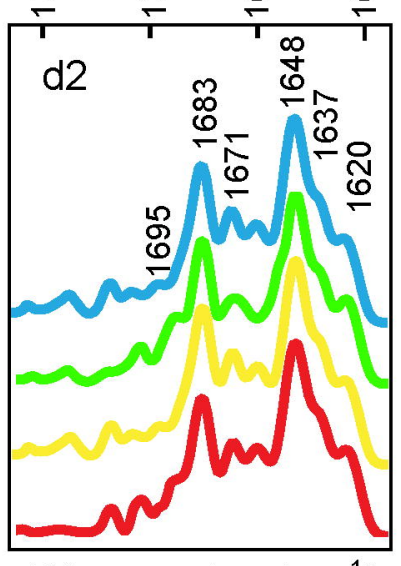

Wavenumber $\left(\mathrm{cm}^{-1}\right)$
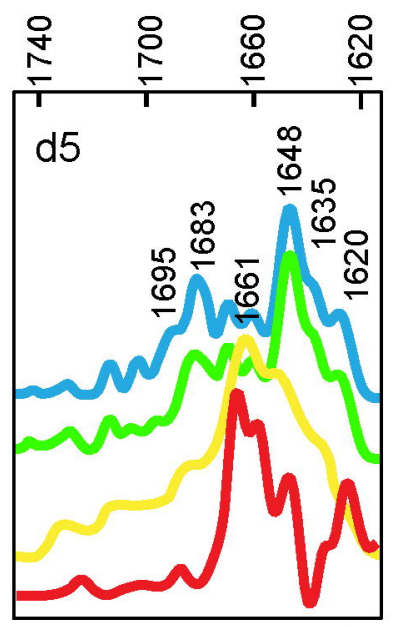

Wavenumber $\left(\mathrm{cm}^{-1}\right)$

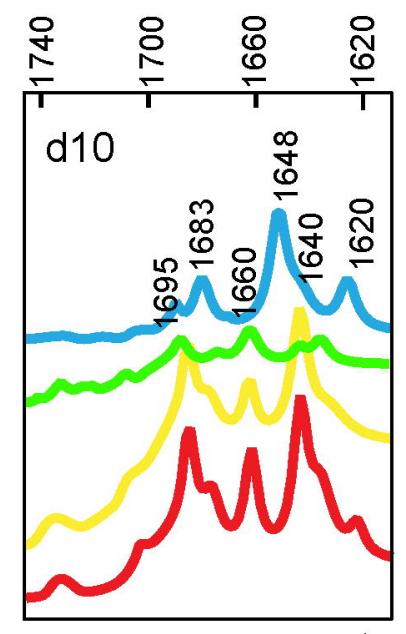

Wavenumber $\left(\mathrm{cm}^{-1}\right)$

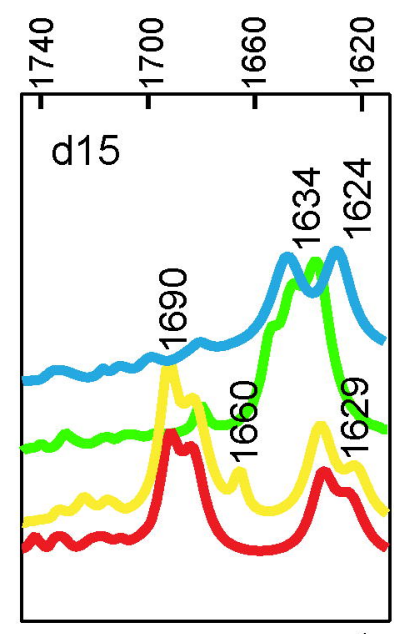

Wavenumber $\left(\mathrm{cm}^{-1}\right)$

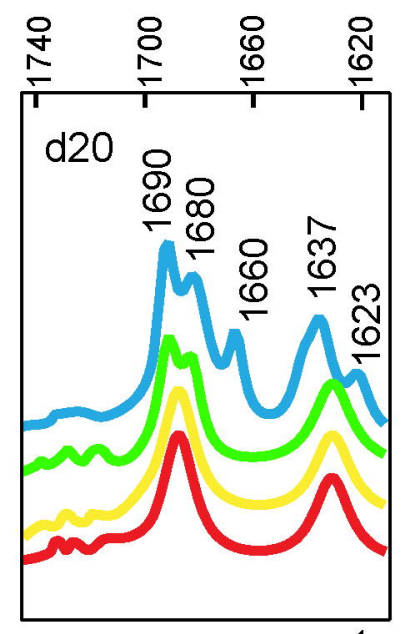

Wavenumber $\left(\mathrm{cm}^{-1}\right)$

d

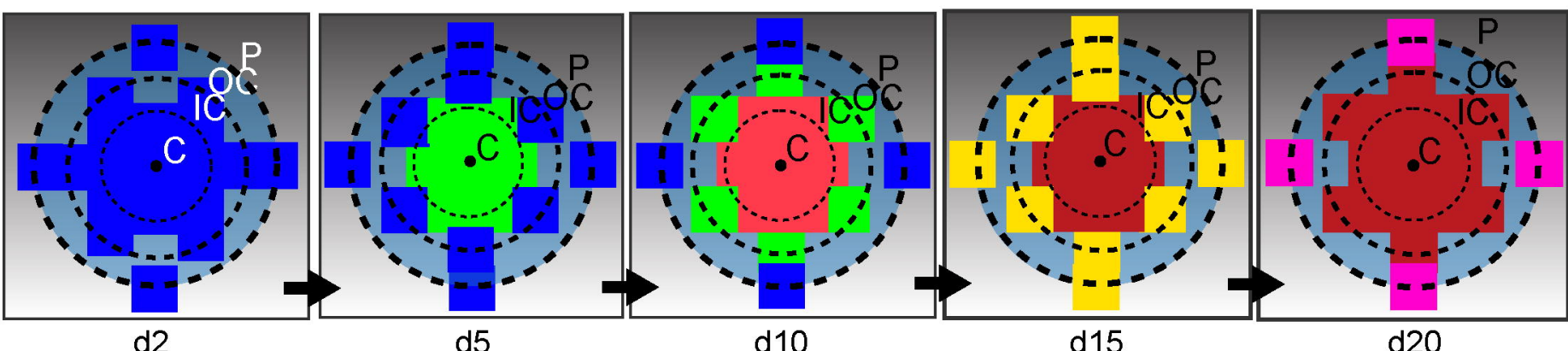

d5

$R C+\beta$-sheet

Cross $\beta+\beta$-sheet

Cross $\beta+\alpha$-Helix 
$\mathbf{a}$

b

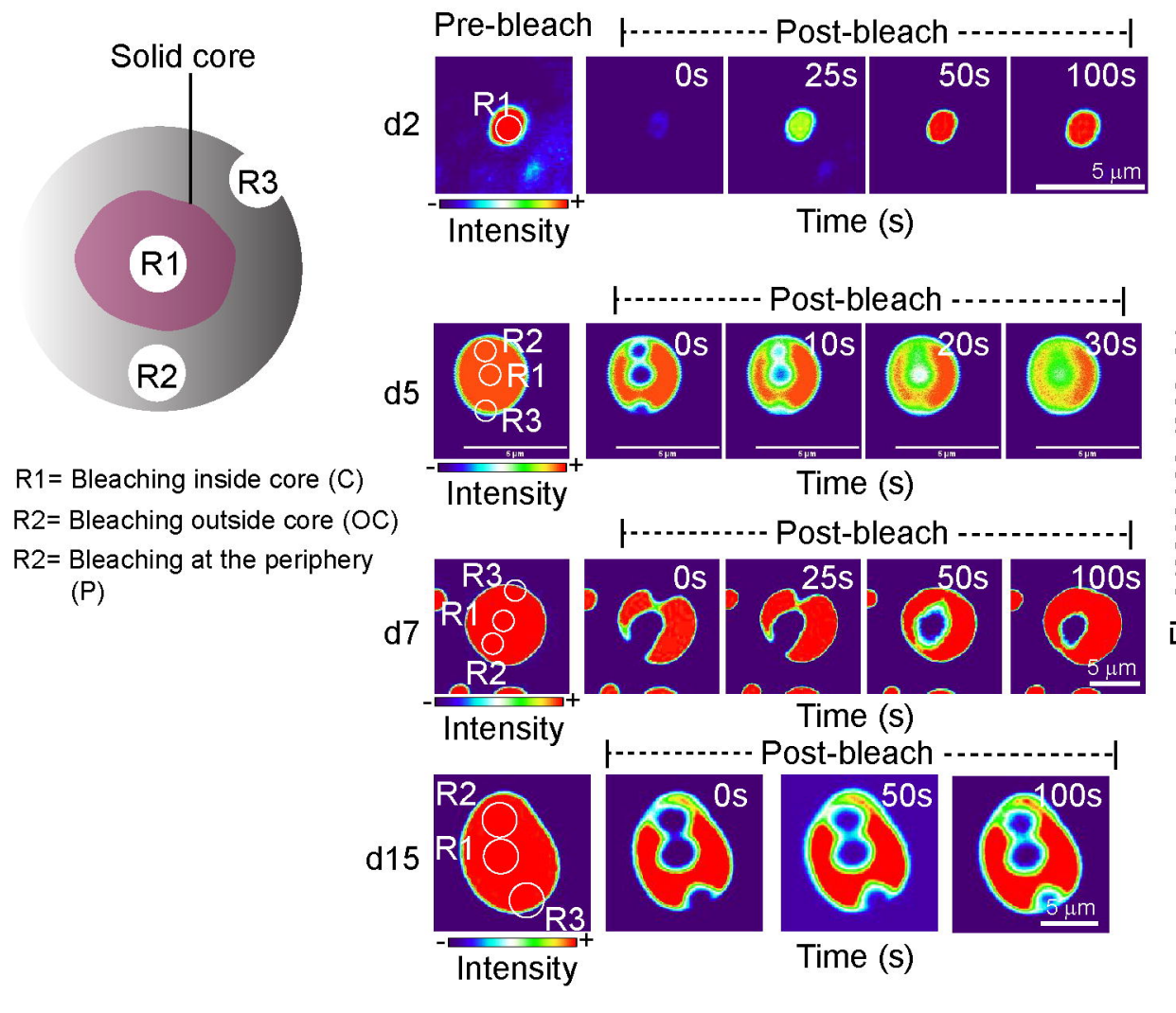

c

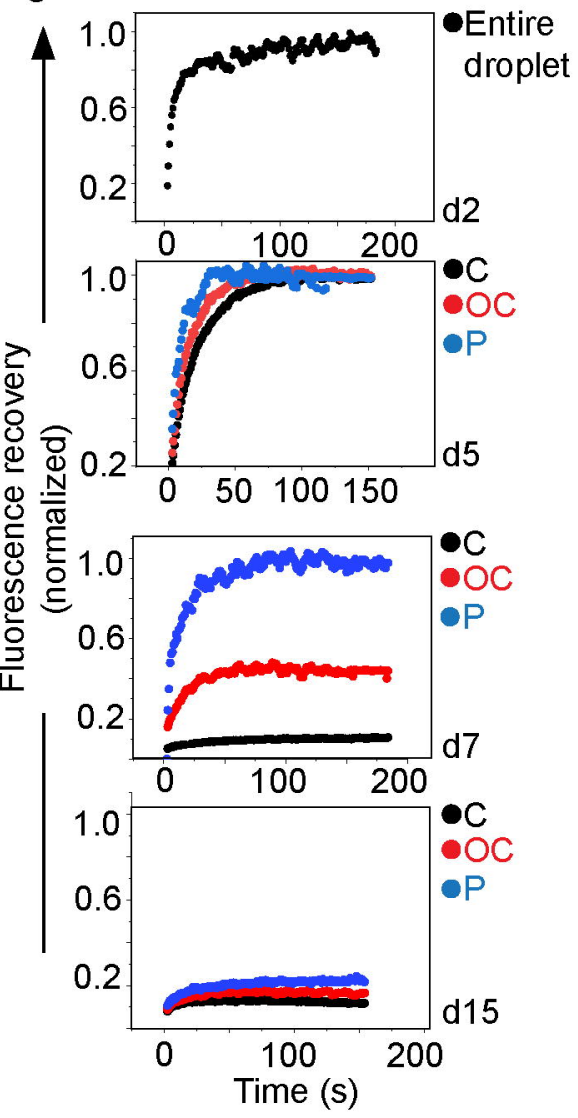


a
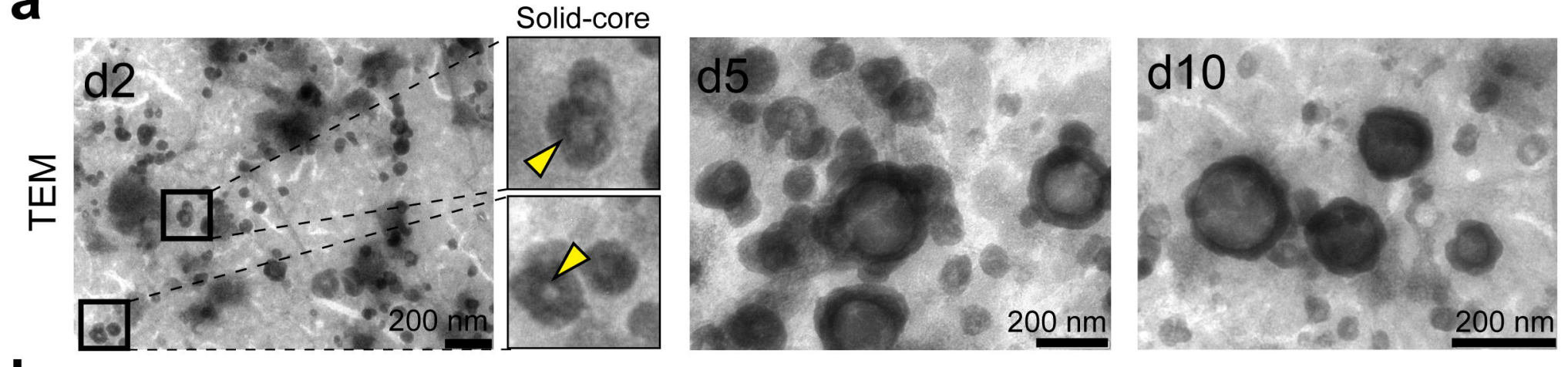

d15

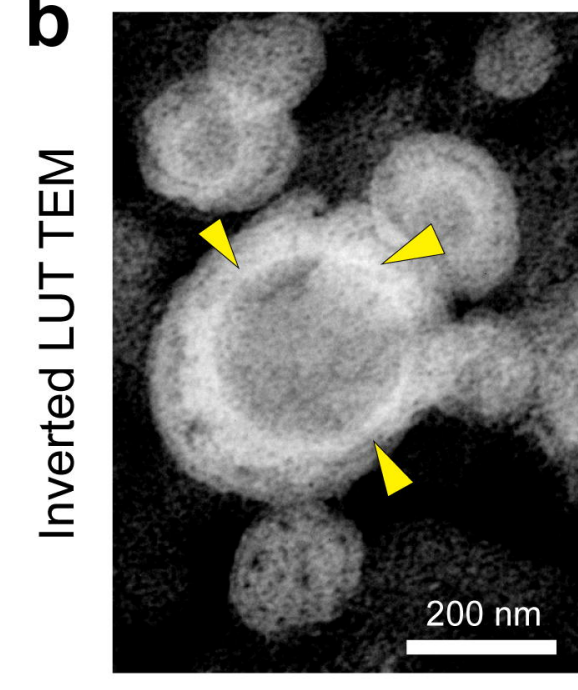

C

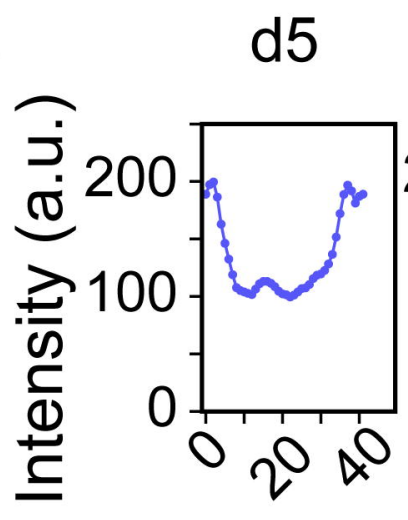

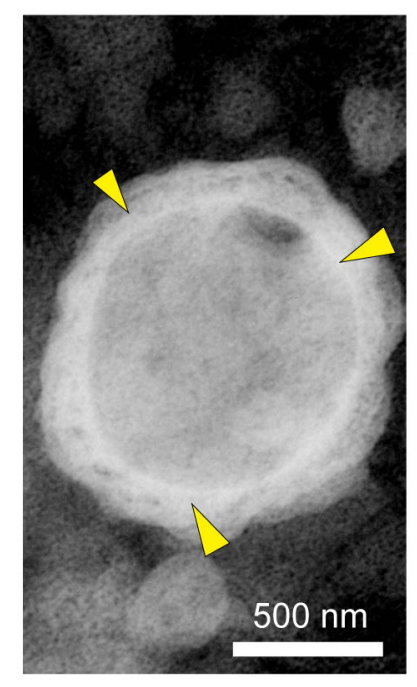

d10

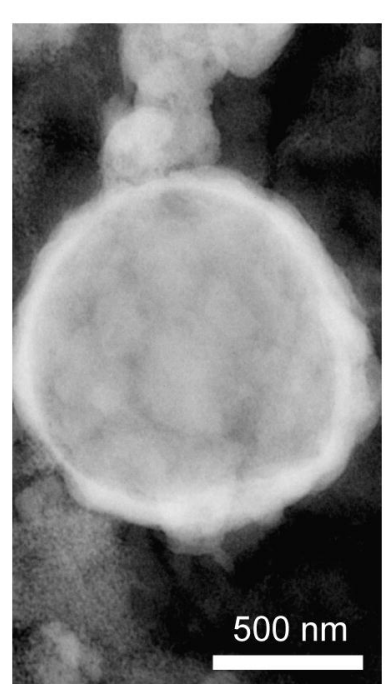

d15

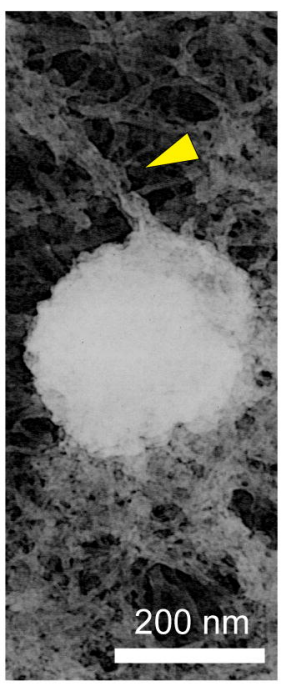

d25 d
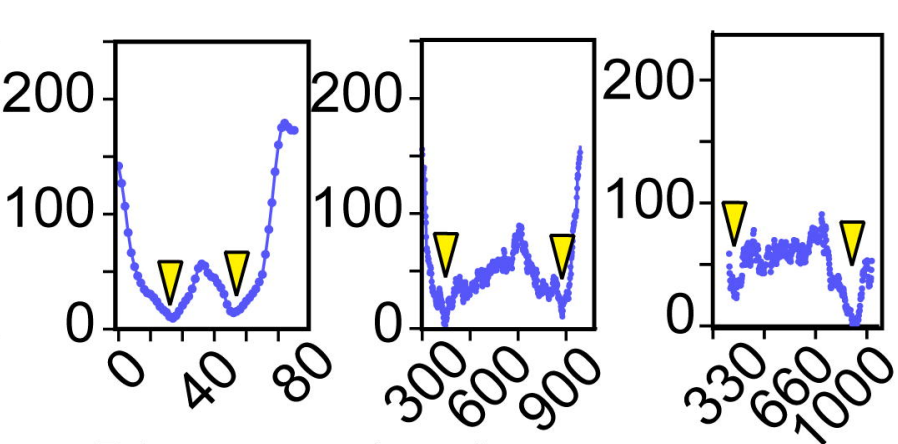

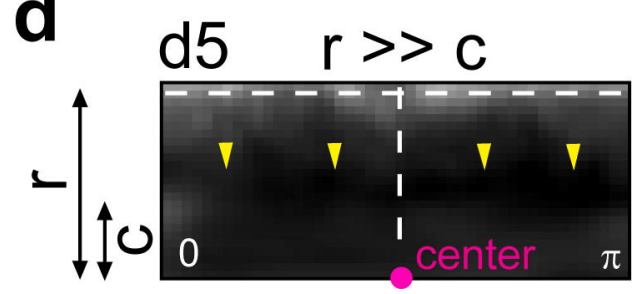

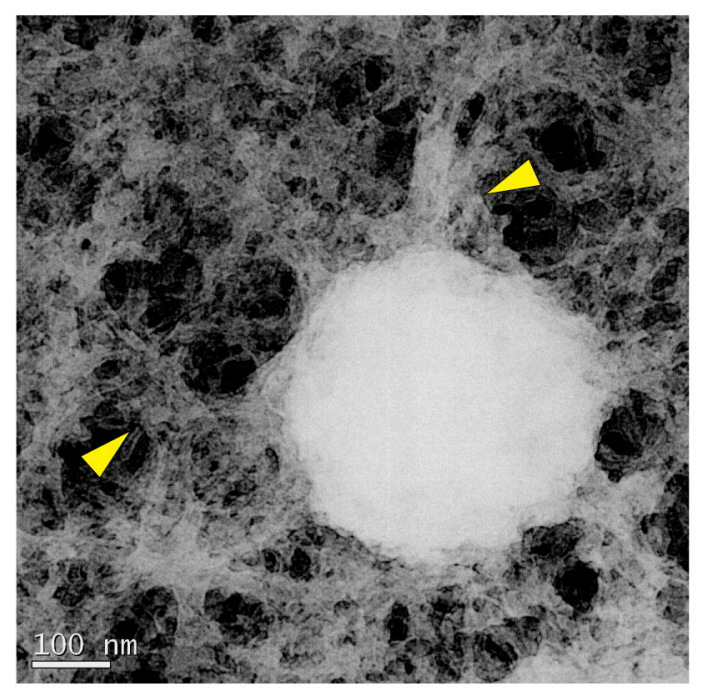

d28

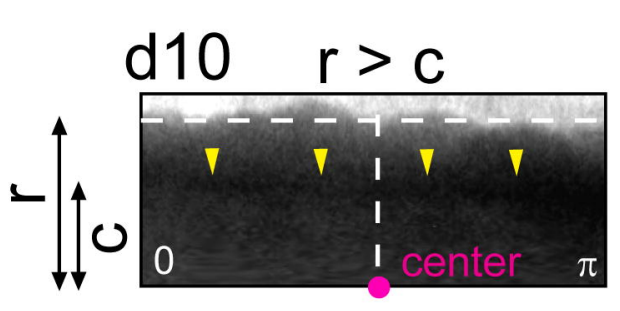

Time $\longrightarrow$

Distance $(\mathrm{nm})$

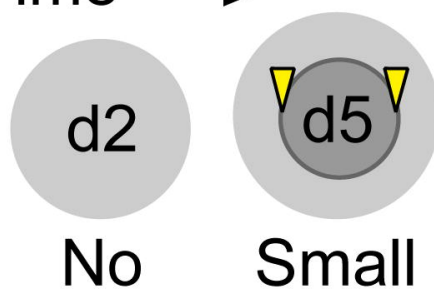
core core

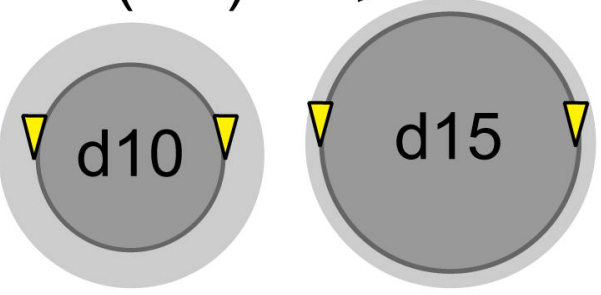

Large Core reaches core periphery

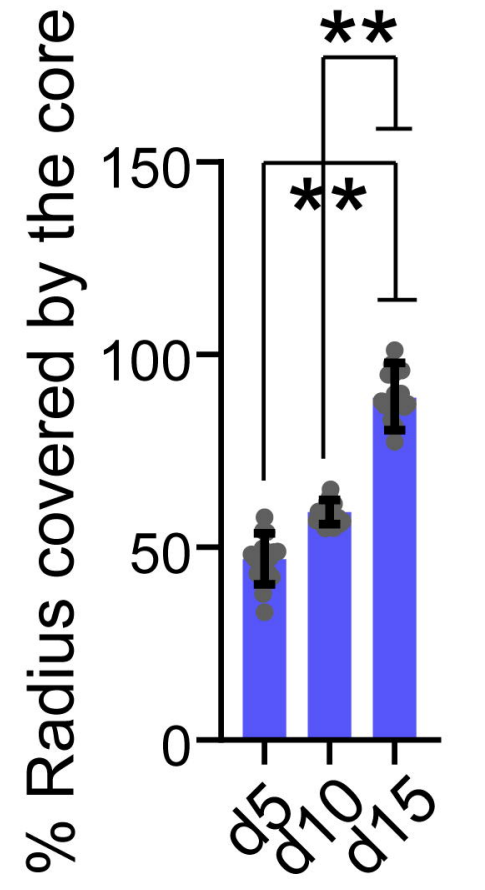




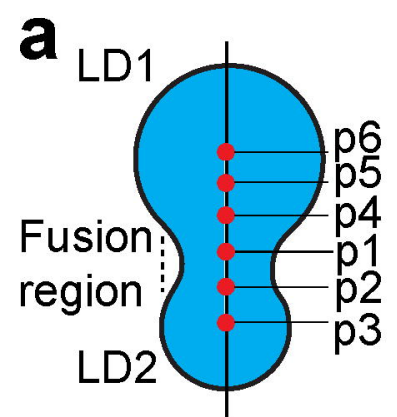

C Fusion axis

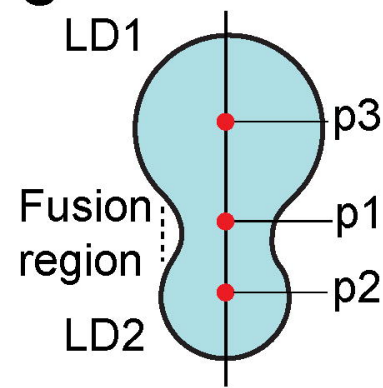

Fusion axis

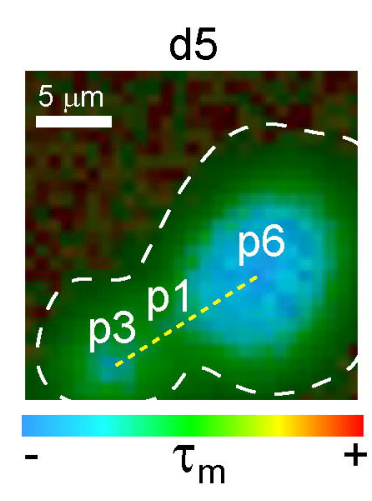

b

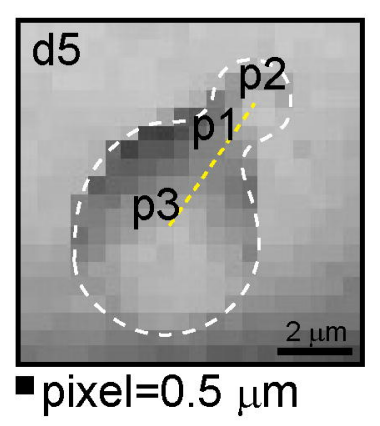

e

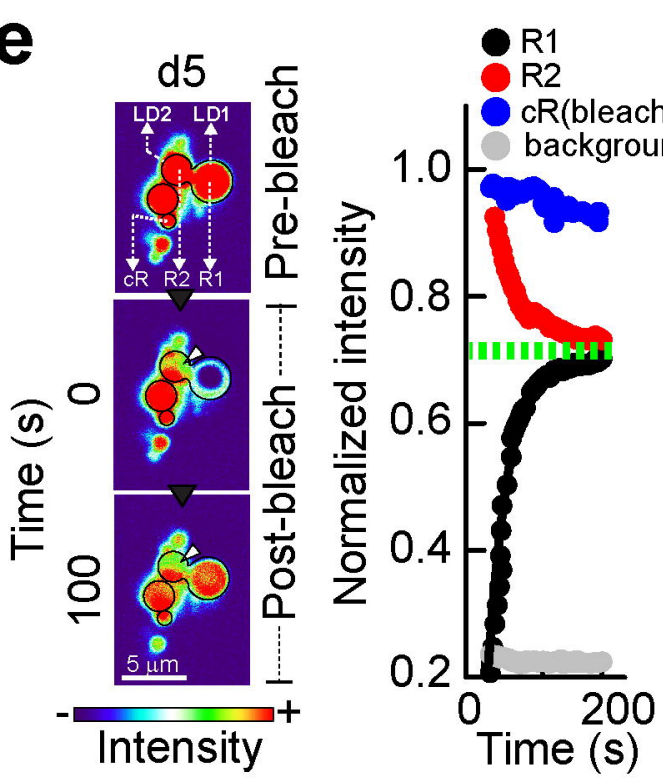

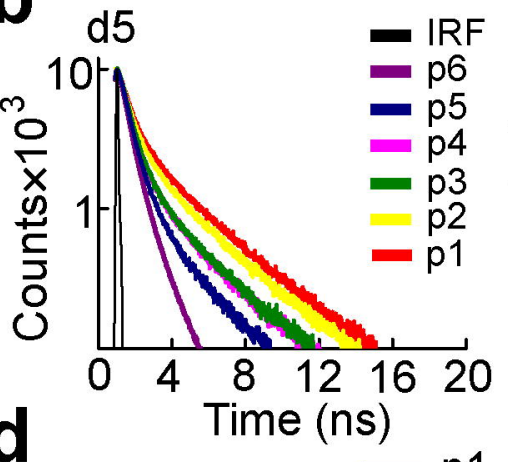

d
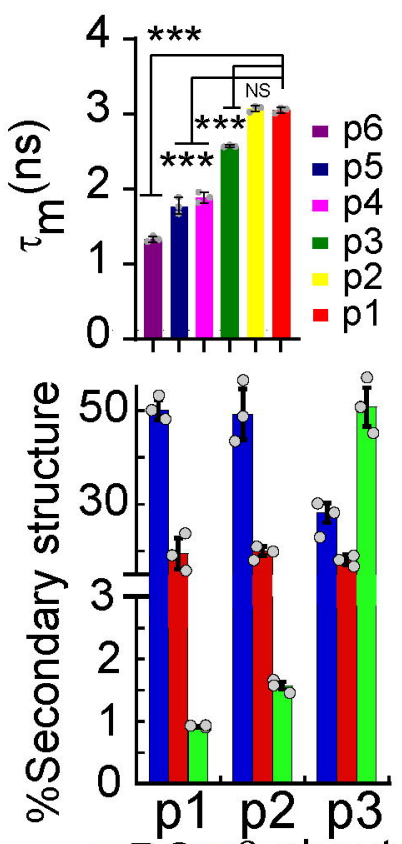

$17001650 \quad 1600$ Wavenumber $\left(\mathrm{cm}^{-1}\right)$
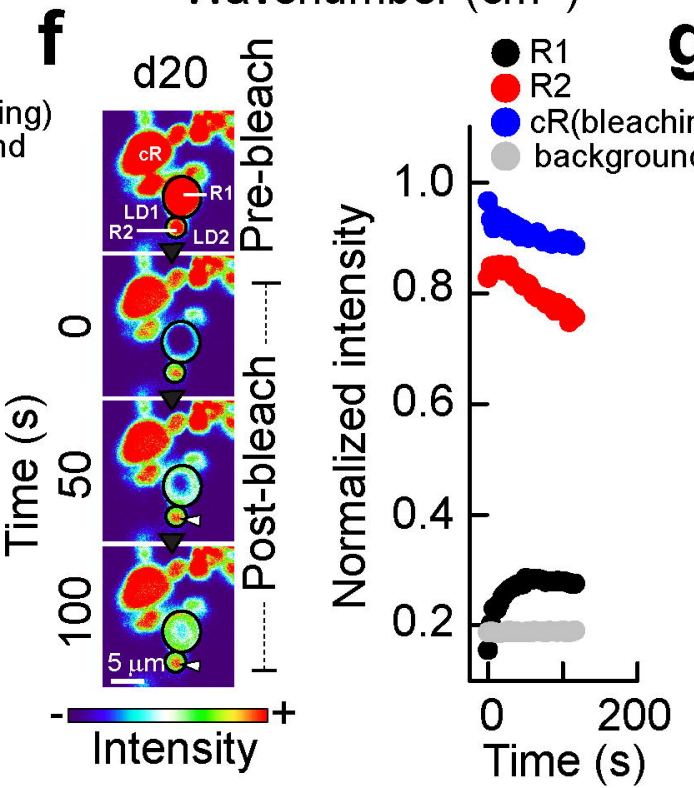

$\square \mathrm{RC}$ - $\beta$-sheet $\alpha$-Helix

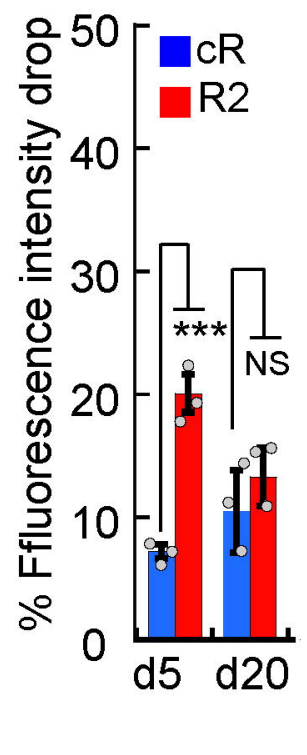




\section{Liquid-to-solid transition}

-

$\theta$

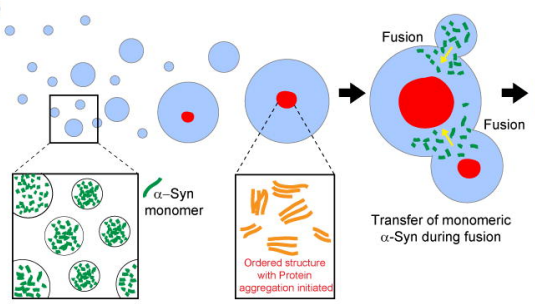

\section{Time}

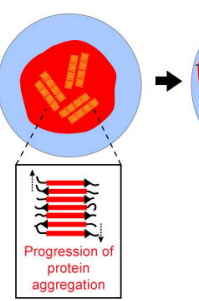

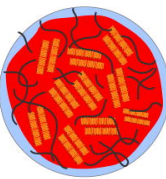

Aggregate core covers the entire droplet with time 
$\alpha-$ synuclein

N-terminal

NAC

C-terminal

$\nabla 61$

MDVFMKGLSK AKEGVVAAAE KTKQGVAEAA GKTKEGVLYV GSKTKEGVVH GVATVAEKTK EQVTNVGGAV VTGVTAVAQK TVEGAGSIAA ATGFVKKDQL GKNEEGAPQE GILEDMPVDP DNEAYEMPSE EGYQDYEPEA

$\Delta 74$ cysteine

$\Delta 95$

b
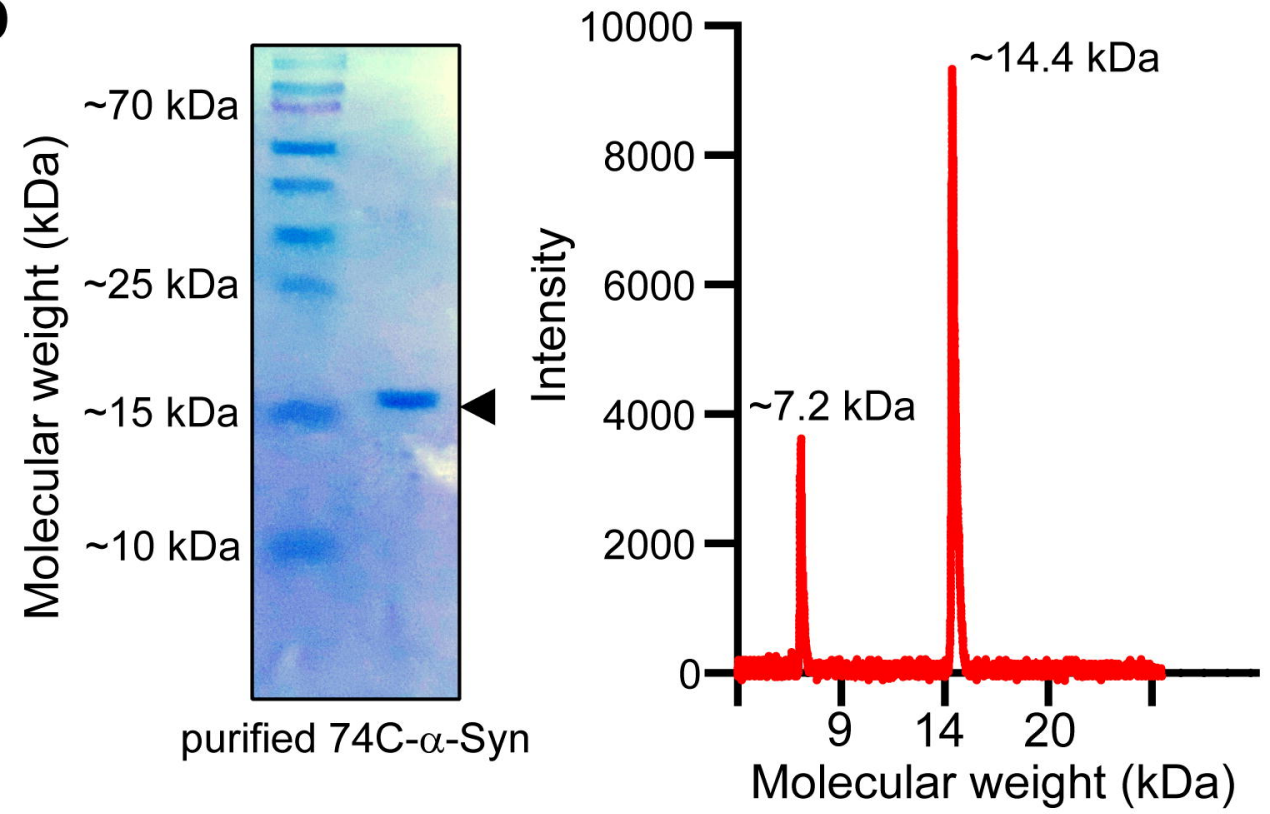
a

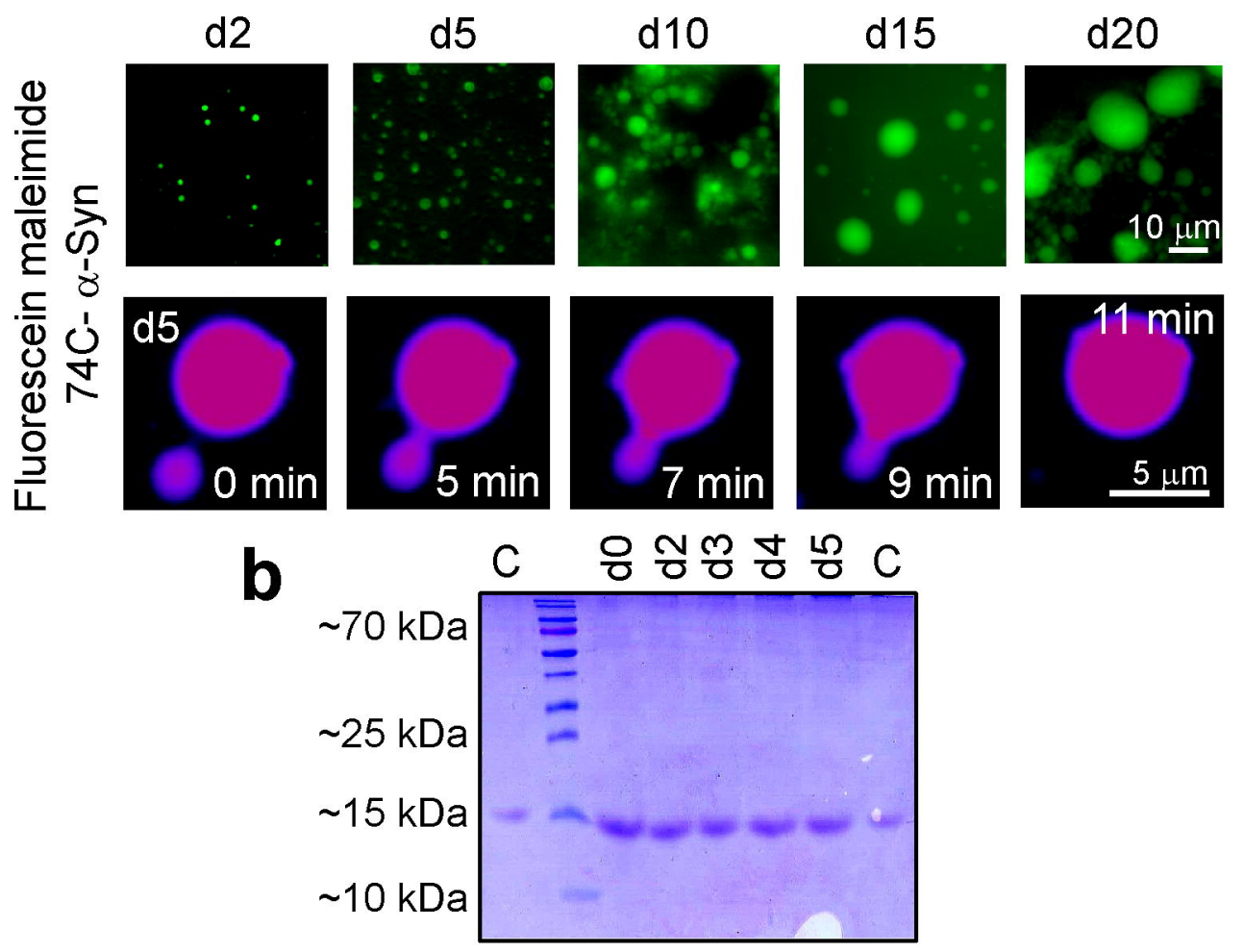


a

b
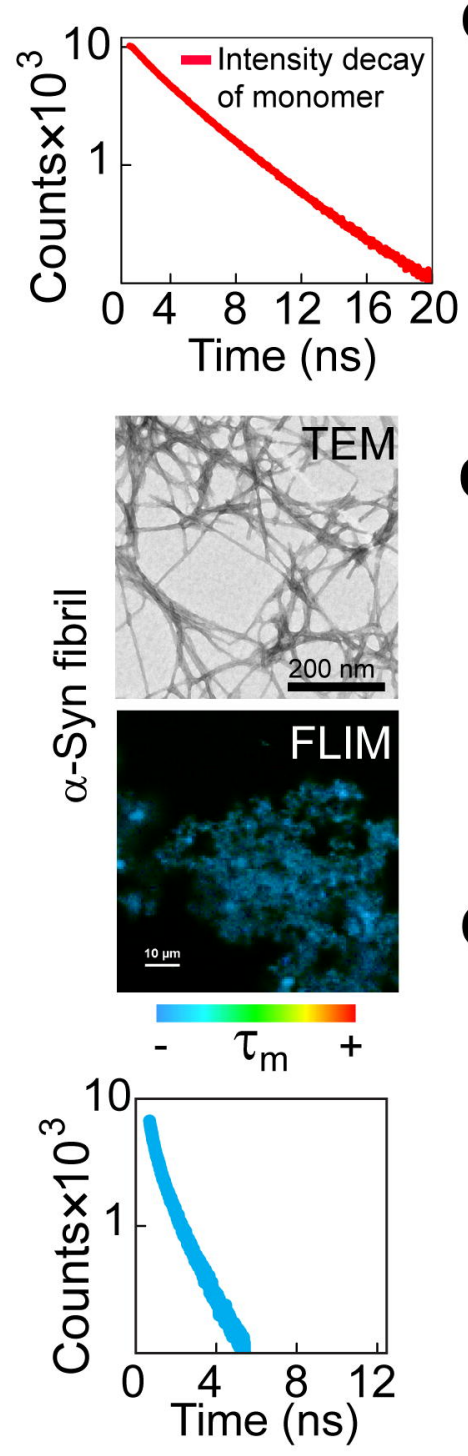

C

d2
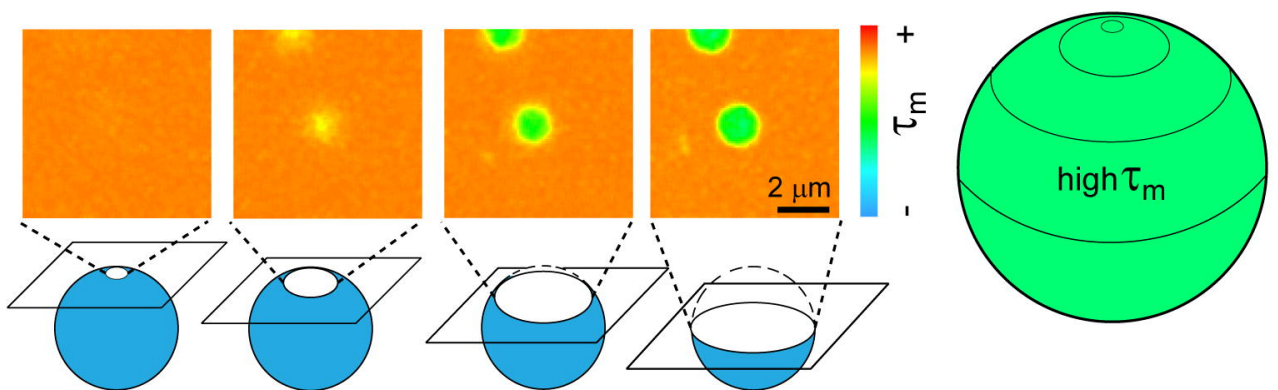

d Plane 1 Plane 2 Plane 3 Central plane
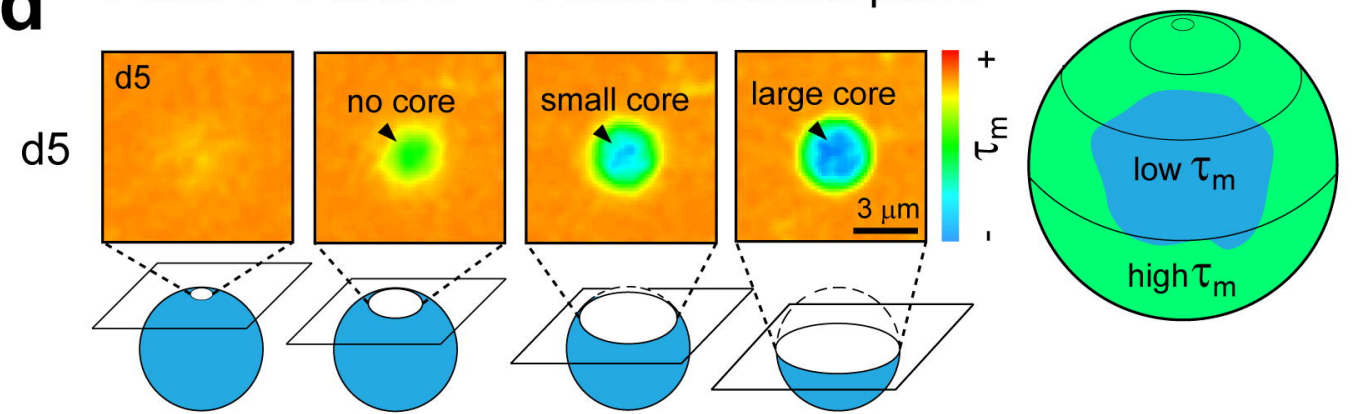

e Plane 1 Plane 2 Plane 3 Central plane

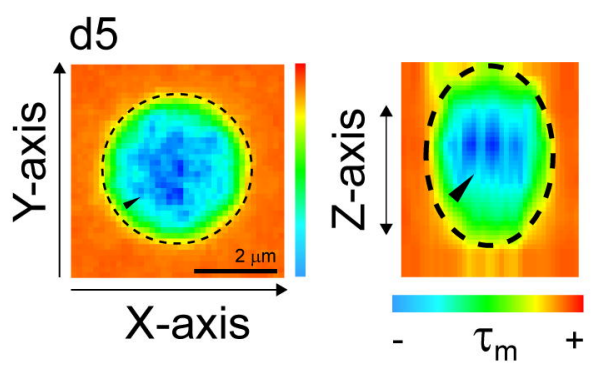




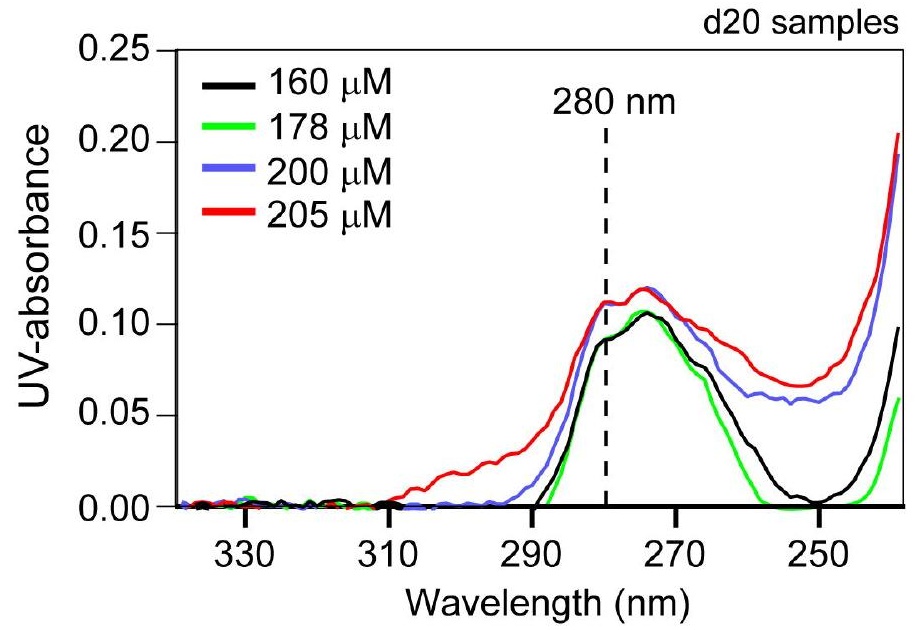



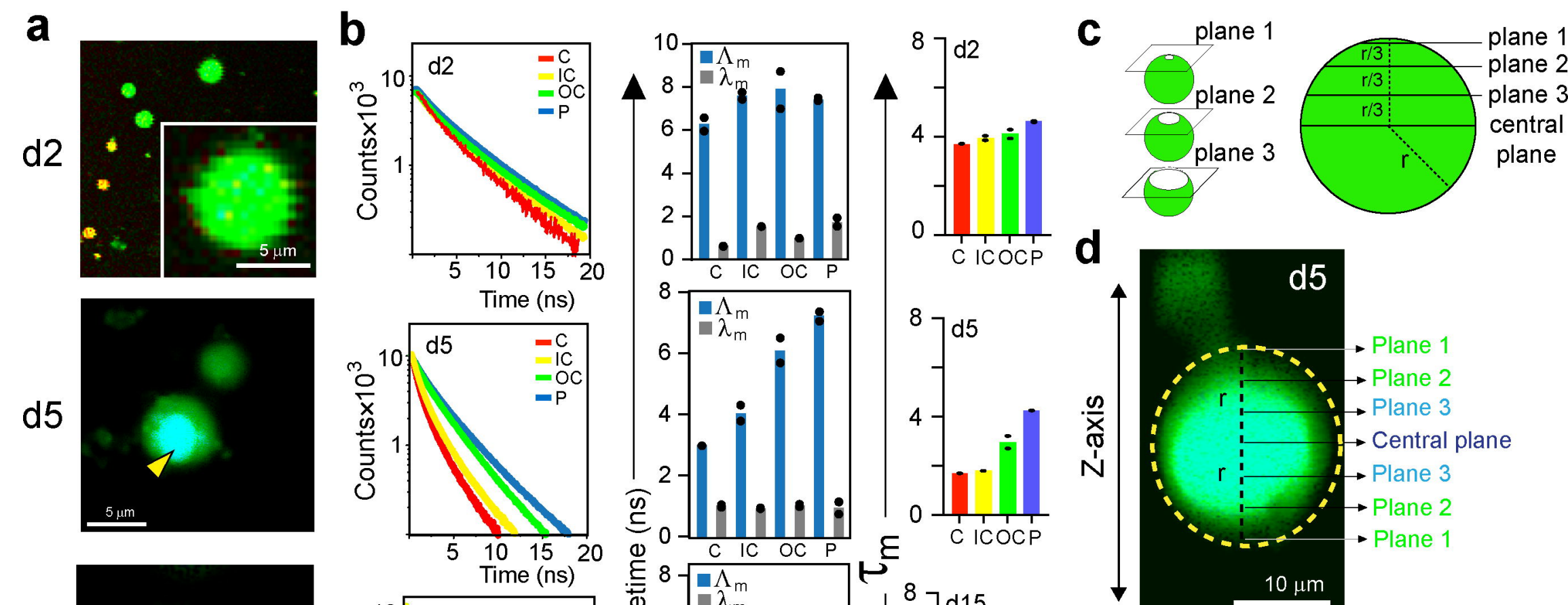

d5
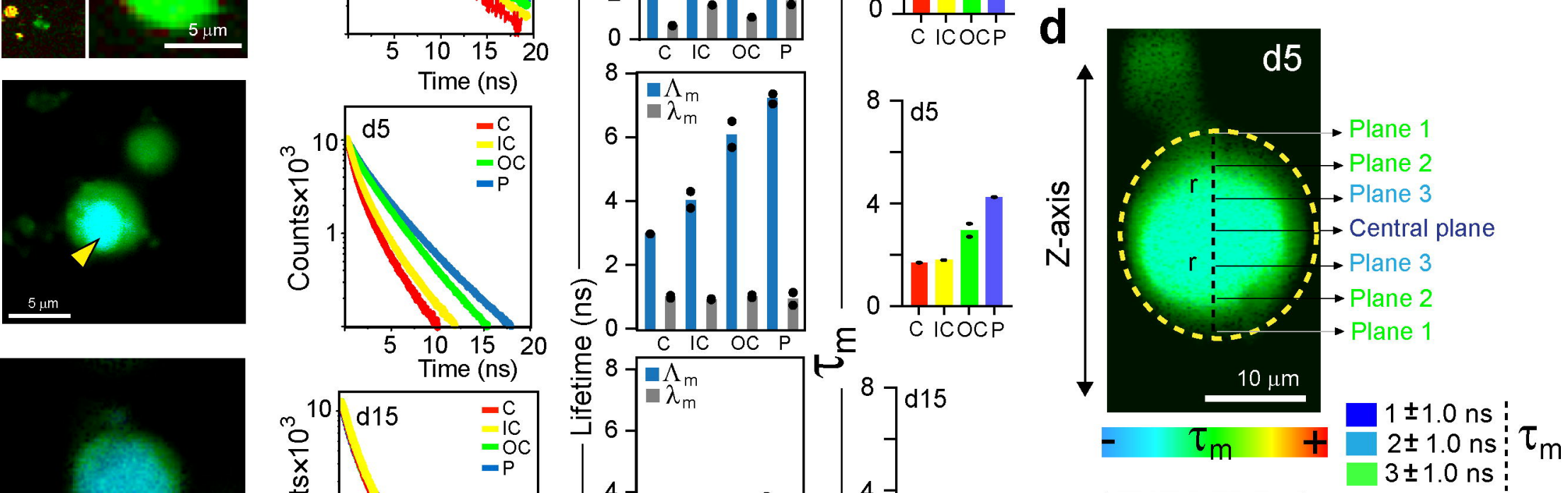

d15
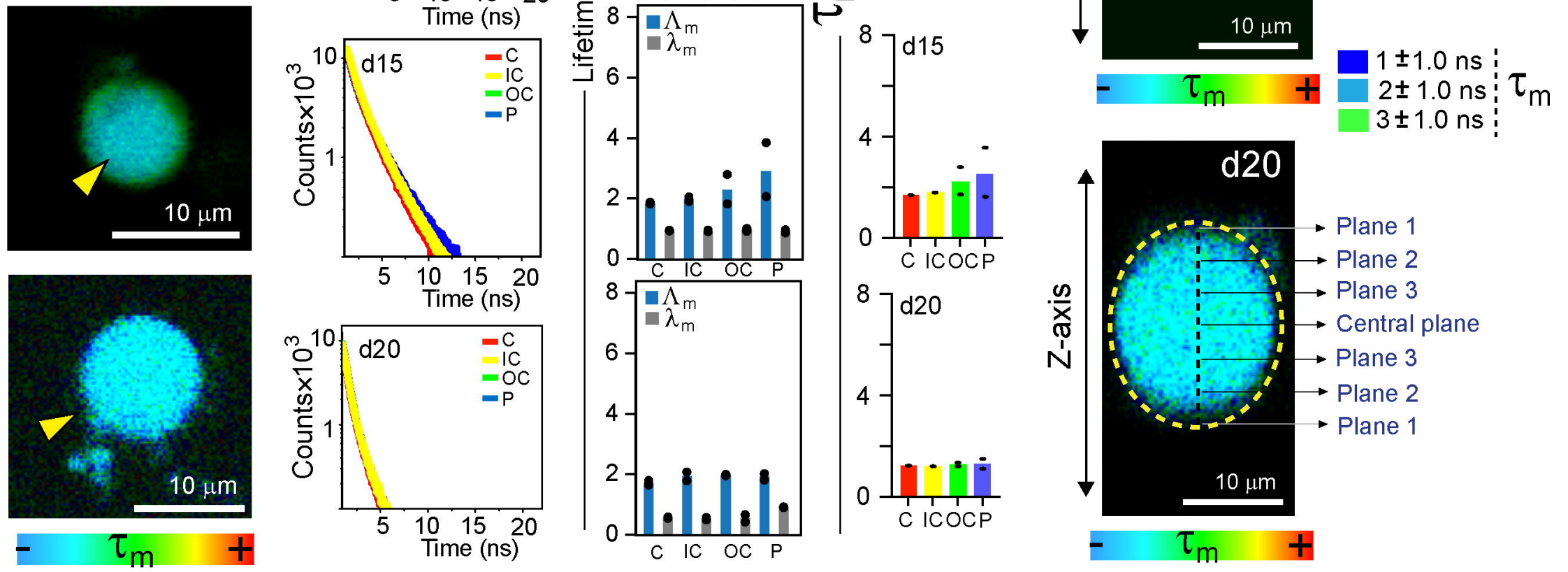

$-\tau_{m}+$
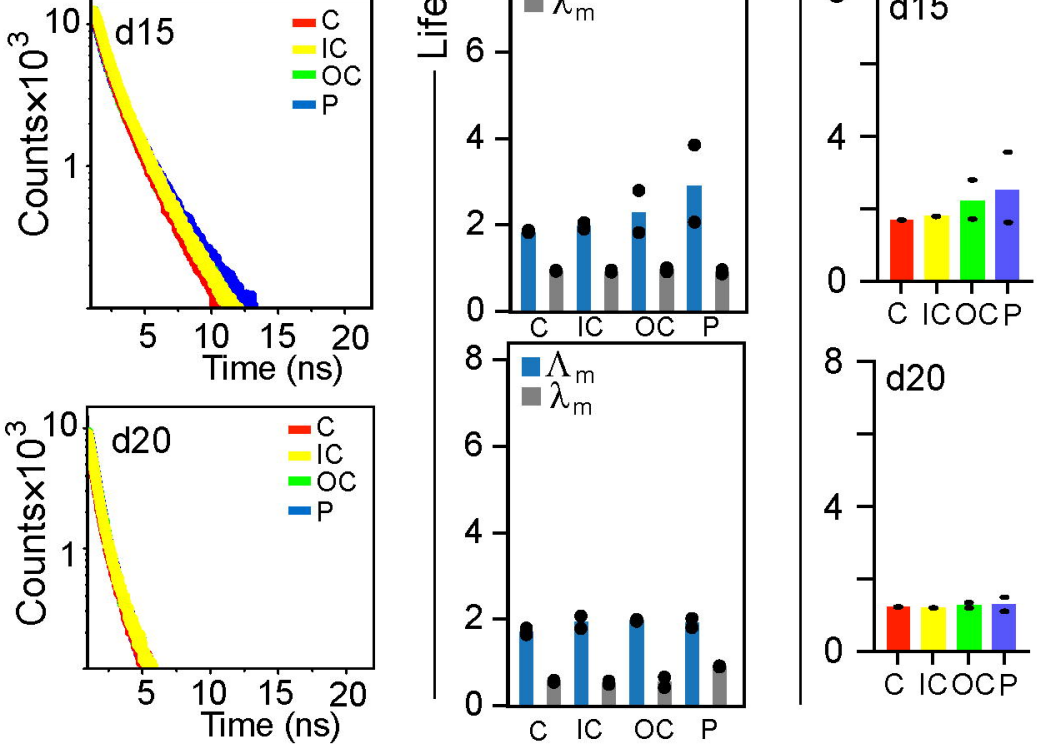


\section{a \\ b Spherical d10 $\alpha$-Syn droplet}

Z-axis

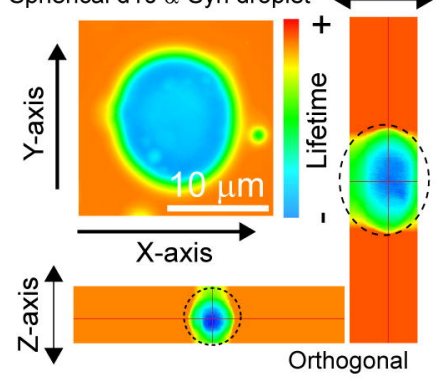

Sedimented d $10 \alpha$-Syn droplet

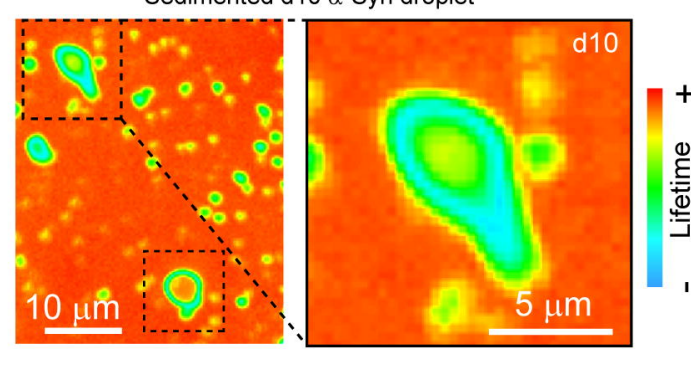




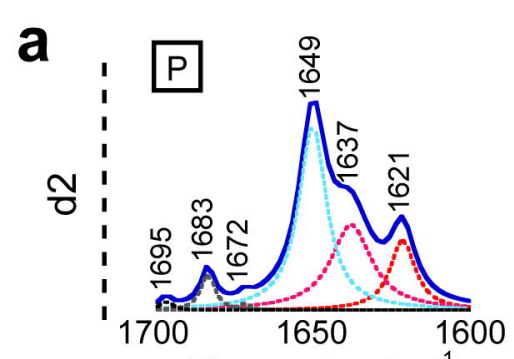

Wavenumber $\left(\mathrm{cm}^{-1}\right)$

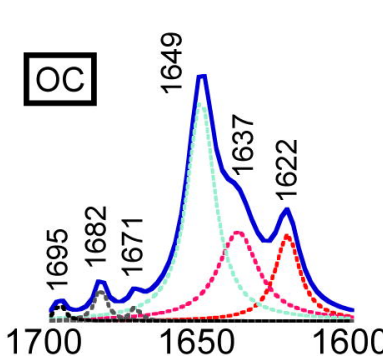

Wavenumber $\left(\mathrm{cm}^{-1}\right)$
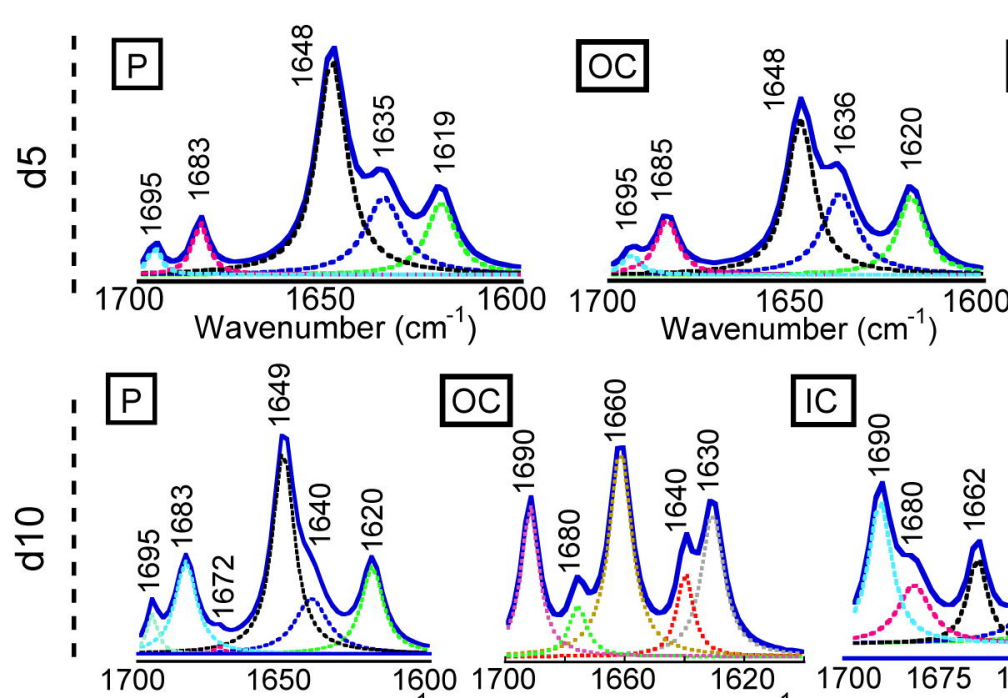

Wavenumber $\left(\mathrm{cm}^{-1}\right)$

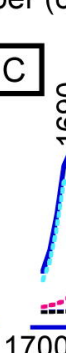

Wavenumber $\left(\mathrm{cm}^{-1}\right)$
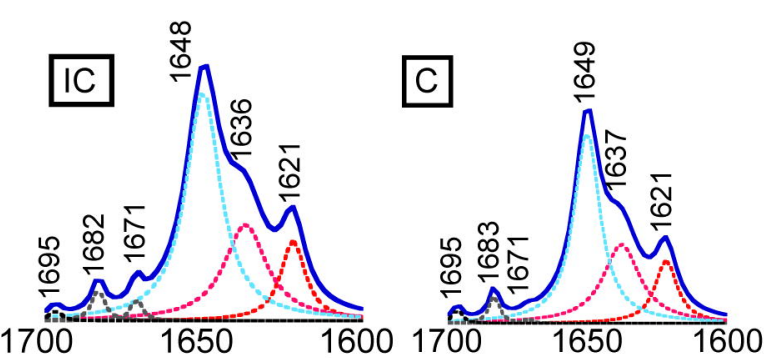

Wavenumber $\left(\mathrm{cm}^{-1}\right)$

Wavenumber $\left(\mathrm{cm}^{-1}\right)$
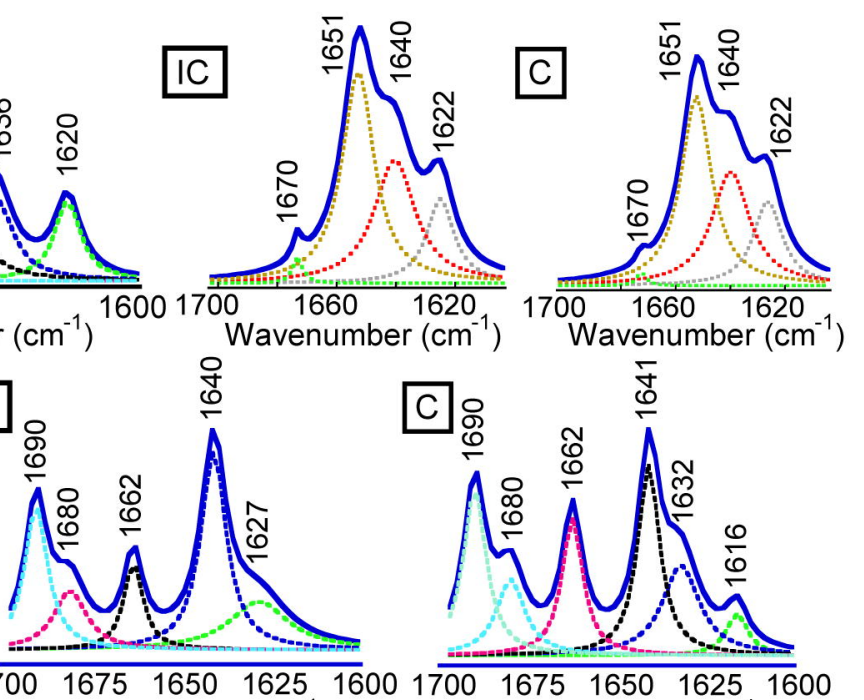

b
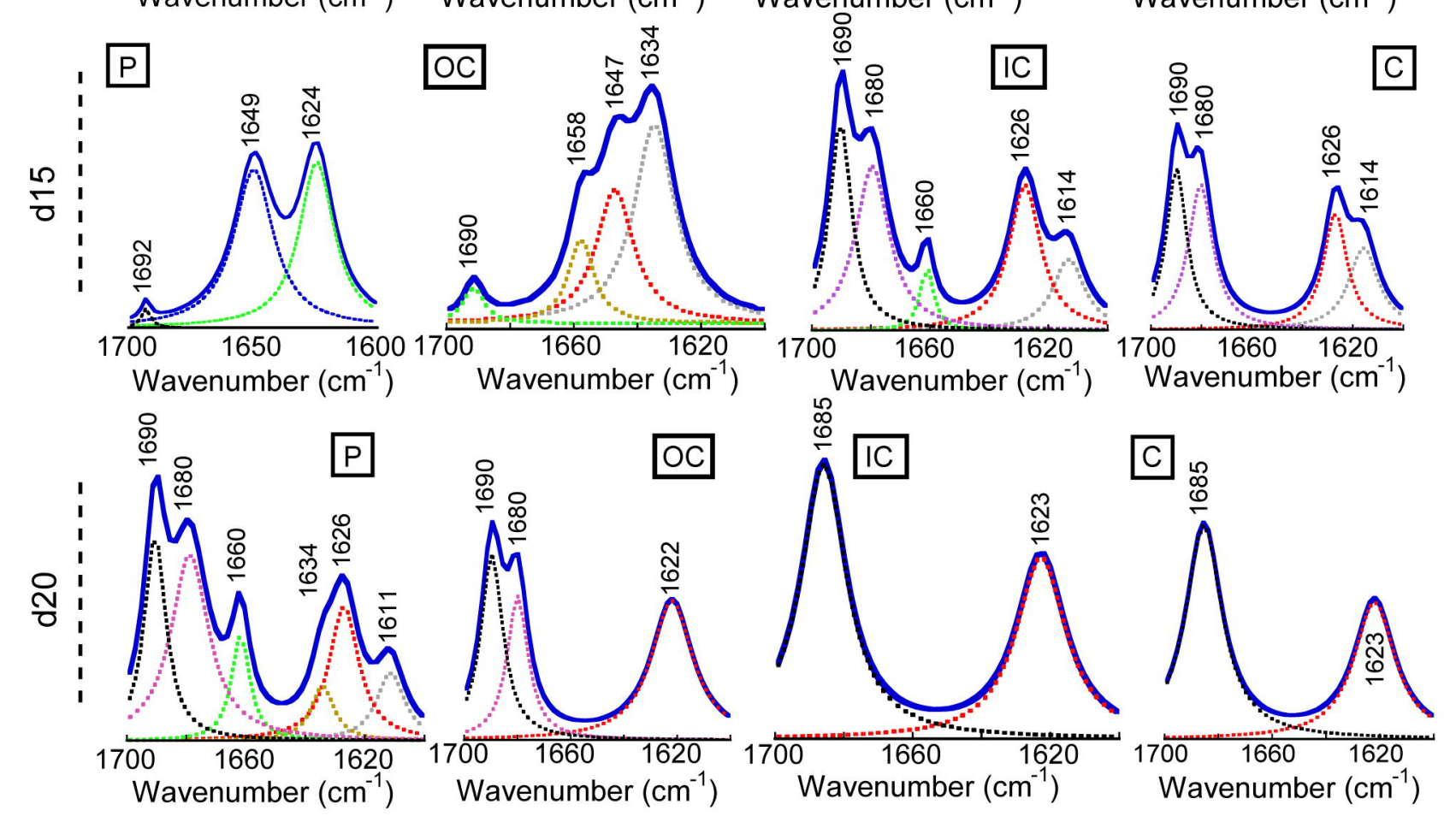
$50 \quad d 2$

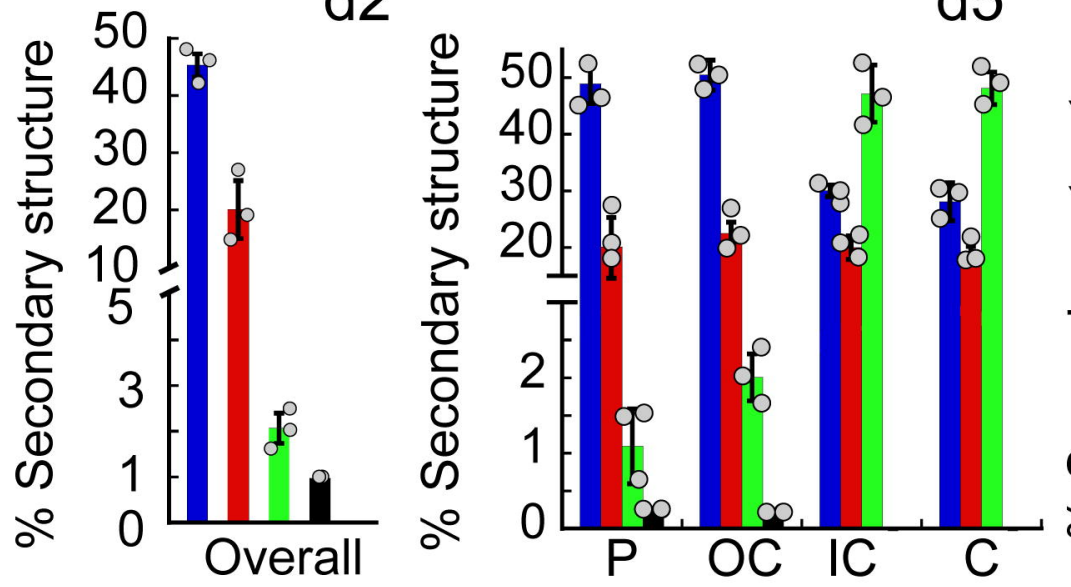

d10

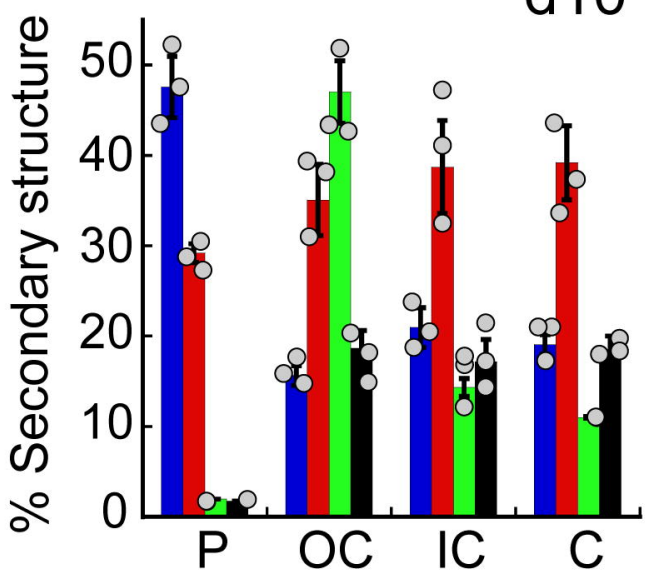
droplet

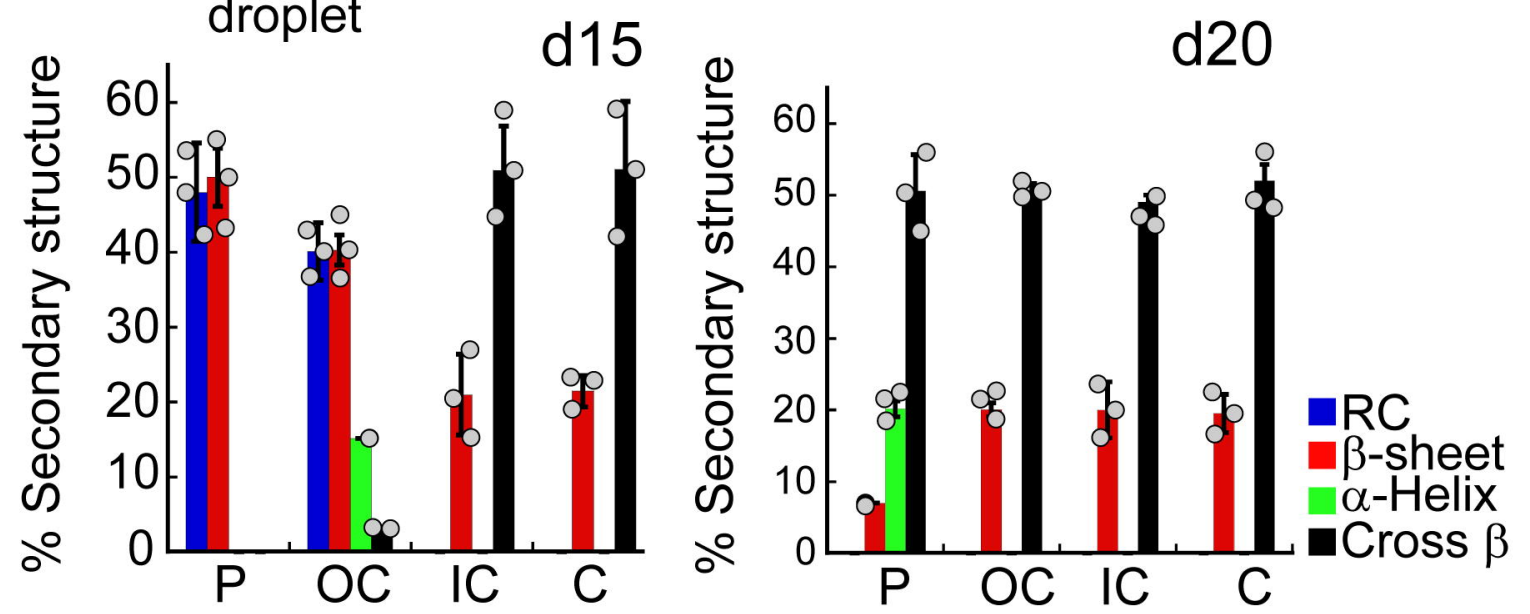


$\alpha$-Syn droplets

\section{Slit}

Objective lens

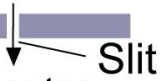

Detector

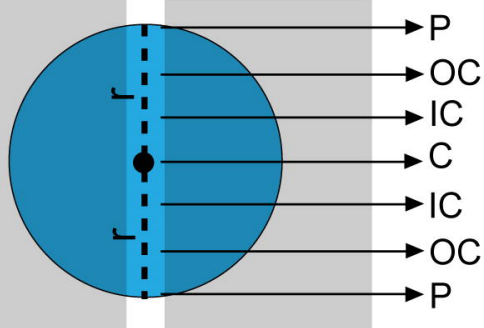

Donor Acceptor emission emission

Zone 1

Zone 2

Zone 3

Zone 4

Zone 5

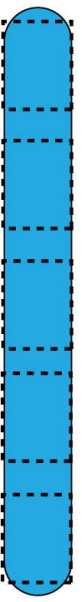

$0^{\text {th }}$ order

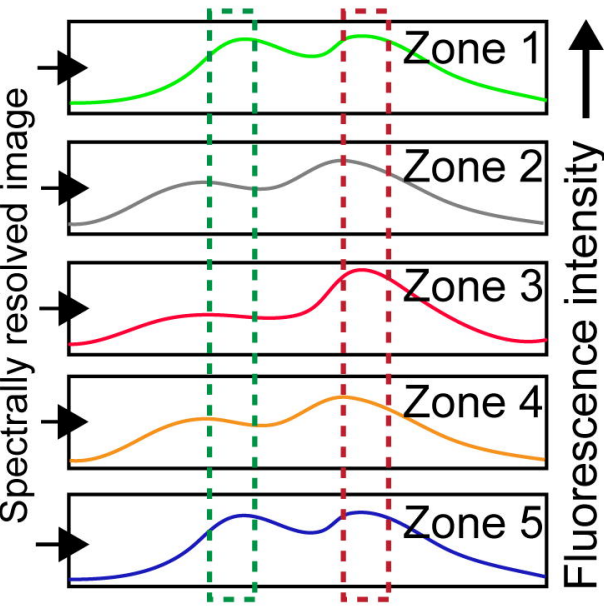

Wavelength $(\mathrm{nm})$ image 


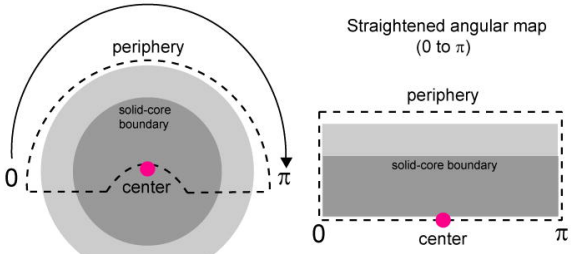



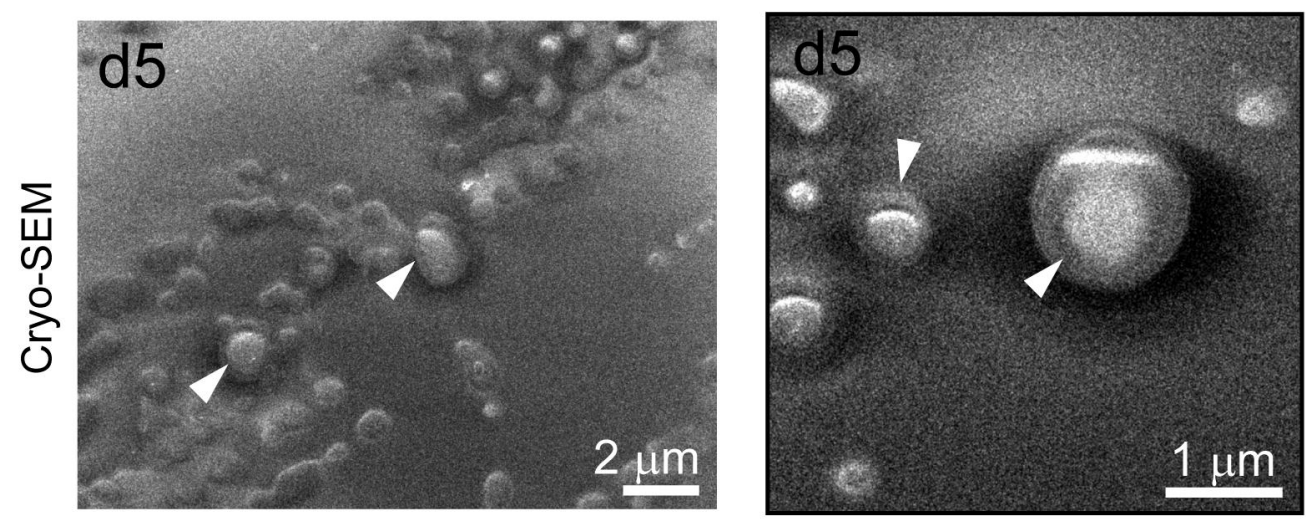